\title{
Influence or selection : dynamics of friendship networks and smoking behavior in adolescence
}

Citation for published version (APA):

Mercken, L. A. G. (2009). Influence or selection : dynamics of friendship networks and smoking behavior in adolescence. [Doctoral Thesis, Maastricht University]. Datawyse / Universitaire Pers Maastricht. https://doi.org/10.26481/dis.20091120lm

Document status and date:

Published: 01/01/2009

DOI:

10.26481/dis.20091120lm

Document Version:

Publisher's PDF, also known as Version of record

\section{Please check the document version of this publication:}

- A submitted manuscript is the version of the article upon submission and before peer-review. There can be important differences between the submitted version and the official published version of record.

People interested in the research are advised to contact the author for the final version of the publication, or visit the DOI to the publisher's website.

- The final author version and the galley proof are versions of the publication after peer review.

- The final published version features the final layout of the paper including the volume, issue and page numbers.

Link to publication

\footnotetext{
General rights rights.

- You may freely distribute the URL identifying the publication in the public portal. please follow below link for the End User Agreement:

www.umlib.nl/taverne-license

Take down policy

If you believe that this document breaches copyright please contact us at:

repository@maastrichtuniversity.nl

providing details and we will investigate your claim.
}

Copyright and moral rights for the publications made accessible in the public portal are retained by the authors and/or other copyright owners and it is a condition of accessing publications that users recognise and abide by the legal requirements associated with these

- Users may download and print one copy of any publication from the public portal for the purpose of private study or research.

- You may not further distribute the material or use it for any profit-making activity or commercial gain

If the publication is distributed under the terms of Article $25 \mathrm{fa}$ of the Dutch Copyright Act, indicated by the "Taverne" license above, 


\section{INFLUENCE OR SELECTION}

Dynamics of friendship networks and smoking behavior in adolescence 


\section{Colophon}

Cover design: Liesbeth Mercken

Production: Datawyse | Universitaire Pers Maastricht

(C) Liesbeth Mercken, Maastricht 2009

ISBN: 9789052788784

The studies presented in this dissertation were performed within the School of Public Health and Primary Care (CAPHRI) which participates in the research school CARE, Netherlands School of Primary Care Research, accredited by the Royal Netherlands Academy of Arts and Sciences. The research presented in this dissertation was supported by a grant of the Netherlands organization for Scientific Research (NWO, Grant No. 401-01-555).

All rights are reserved. No part of this book may be reproduced or transmitted in any form or by any means, without the written permission from the author or, where appropriate, the publisher of the article. 


\section{INFLUENCE OR SELECTION}

Dynamics of friendship networks and smoking behavior in adolescence

\section{PROEFSCHRIFT}

Ter verkrijging van de graad van doctor aan de Universiteit Maastricht, op gezag van Rector Magnificus, Prof.mr. G.P.M.F. Mols, volgens het besluit van het College van Decanen, in het openbaar te verdedigen op vrijdag 20 november 2009 om 16.00 uur

door

Liesbeth Ann Gerard Mercken

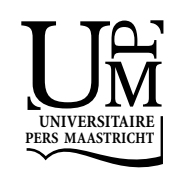




\section{Promotores}

Prof.dr. H. de Vries

Prof.dr. T.A.B. Snijders (University of Oxford, Rijksuniversiteit Groningen)

\section{Copromotor}

Dr. M. Candel

\section{Beoordelingscommissie}

Prof.dr. N.K. de Vries (voorzitter)

Prof.dr. M. Berger

Dr. W. Burk (Radboud Universiteit Nijmegen)

Prof.dr. L. Lechner (Open Universiteit Nederland)

Prof.dr.ir. C.P. van Schayck 


\section{CONTENTS}

Chapter 1 General introduction 7

$\begin{array}{lll}\text { Chapter } 2 & \text { Disentangling selection and influence effects }\end{array}$

$\begin{array}{lll}\text { Chapter } 3 & \text { Changes during early and mid adolescence } & 37\end{array}$

$\begin{array}{lll}\text { Chapter } 4 & \text { Social network analyses in six European countries } & 57\end{array}$

$\begin{array}{lll}\text { Chapter } 5 & \text { A social network analyses in a Finnish sample } & 75\end{array}$

Chapter 6 Selection and influence in gender segregated networks 99

$\begin{array}{lll}\text { Chapter } 7 & \text { General discussion } & 117\end{array}$

Summary $\quad 131$

Samenvatting 135

References 139

Dankwoord 153

$\begin{array}{ll}\text { Curriculum Vitae } & 157\end{array}$ 



\section{CHAPTER 1}

General introduction 
Tobacco use is the second major global cause of mortality and the fourth most common risk factor for disease worldwide [1]. In most Western countries, there is an increase during adolescence in the prevalence of smoking [2, 3]. Among 13-year olds, the prevalence of regular smoking varies from $3.5 \%$ to $12.5 \%$ and increases to $17 \%$ to $24.5 \%$ for 15 -year olds [4]. Experimenting with smoking by adolescents is not without risks. Once adolescents start to smoke, it is very hard to quit and due to the physiological dependence on nicotine they are more likely to become a regular smoker [5-7]. Insights into the different processes that might explain smoking initiation and maintenance during adolescence may benefit the development of prevention programs and adaptation of policies regarding effective smoking prevention.

Peer groups play a crucial role in adolescent smoking behavior [8-11], as evidenced by previous research suggesting that smokers befriend smokers and nonsmokers befriend non-smokers [9, 10]. Similarity in smoking behavior among friends might be caused by social influence processes or selection of friends based on similar smoking behavior. This dissertation aims to disentangle selection and influence processes in the context of adolescent smoking behavior.

\section{SMOKING BEHAVIOR SIMILARITY AMONG FRIENDS}

A substantial body of research suggests that smoking behavior is often similar among friends $[9,10]$. Similarity in smoking behavior among friends may occur as a result of two different processes: friendship selection and influence processes.

Selection occurs when adolescents select friends with similar smoking behavior during the formation of a new friendship [12, 13]. When smokers select smoking friends and non-smokers select non-smoking friends, smoking behavior among friends becomes similar. Smoking behavior similarity among friends may furthermore be increased by deselecting dissimilar smoking friends [13].

Social influence among friends occurs when adolescents adjust their smoking behavior in response to the example of or the direct pressure by their friends [12-14]. According to Social Learning Theory, individuals observe, model, and imitate behavior of important individuals in their environment $[15,16]$. As best friends set an example or function as a role model, adolescents are inclined to imitate their best friends' smoking behavior. Adolescents might also perceive direct pressure to smoke. Direct pressure encompasses behaviors like offering a cigarette, persuasion, challenging or pestering someone to smoke [17]. Furthermore, there are several other 
influence processes that may cause smoking behavior similarity among friends. Friends for example may be simultaneously exposed to external influences from parents and siblings, the neighborhood, school policies or the media [18-22]. Figure 1 demonstrates how smoking behavior similarity among friends might be caused by selection and influence processes.

Figure 1 Selection and influence processes leading to similarity among friends

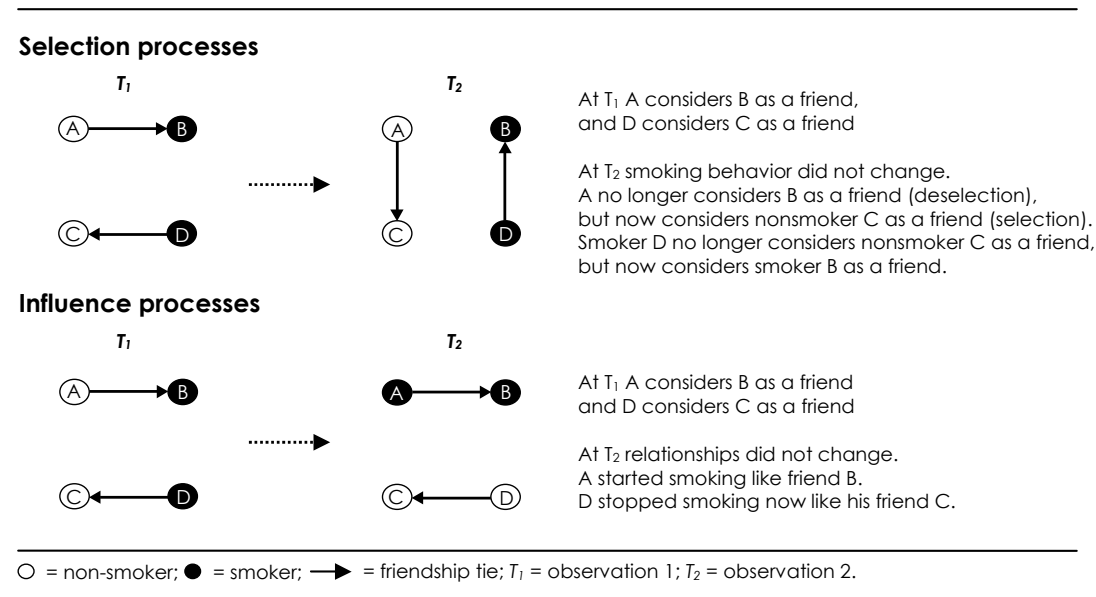

\section{PREVIOUS RESEARCH}

During the past few decades, several studies have tried to separate selection from influence processes in adolescent deviant behaviors such as smoking behavior, marijuana use, alcohol consumption and delinquency. Cohen (1977) examined the relative contribution of influence, selection or deselection to group homogeneity according to 18 individual characteristics, including smoking behavior. The results showed that both influence and selection contributed to homogeneity. Initial selection of similar friends accounted for most of the similarity observed in any given group [23]. Kandel (1978) focused on the degree of similarity on a variety of characteristics within real-life adolescent friendships in New York high schools. Similarity was lowest in unstable friendships, both prior to and after dissolution of the friendship. Influence and selection contributed equally to the similarity observed cross-sectional on the four characteristics, marijuana use, educational aspirations, political orientation and minor delinquency [24]. Fisher and Bauman [12] conducted 
a longitudinal study that investigated cigarette-smoking behavior of ninth graders. Changes in the composition of friendship pairs over time were examined. They concluded that selection was stronger than influence for smoking behavior. The deselection mechanism received negligible support in this study. Ennett and Bauman [13] reanalyzed the same data after creating peer groups with social network techniques. They concluded that selection appeared to be at least equally important as influence in peer group homogeneity. Therefore, peer influence will be exaggerated if one does not control for selection. Kirke [25] interviewed all 14 to 18 year old adolescents from one district electoral division in Dublin County Borough (Ireland). Individual level data on the use of alcohol, cigarettes and drugs were combined with the social network data. Findings suggested that peer influence by the peers who are similar would be exaggerated if selection is not controlled for.

If smokers select each other as friends, then still the question remains to be answered why they started to smoke to begin with. Engels and colleagues [26] demonstrated that both influence and selection processes contributed to peer group homogeneity, but the largest part had to be attributed to selection. Urberg and colleagues [27] proposed a two-stage model of peer influence. Selection (stage 1) was analyzed across the first two measurements of a four-wave longitudinal dataset. Influence was analyzed using the third and fourth waves of the dataset. Adolescents who did not value school achievement or spending time with parents choose more friends who smoke more cigarettes than they did. High peer acceptance and high friendship quality resulted in adolescents conforming to their friends' substance use behavior. Simons-Morton and Chen [28] found that initial smoking behavior predicted an increase in smoking friends over time, indicating selection, and the initial number of smoking friends predicted smoking progression, providing evidence for influence. De Vries and co-workers [29] conducted a longitudinal study in six European countries, investigating whether smoking onset in young adolescents is predicted by peer or parental smoking using structural equation modeling (SEM) techniques. No support was found for peer smoking as an important predictor for smoking onset. Support was found for peer selection processes.

The main conclusions of the above reviewed body of research, as well as several other studies conducted during the past decade [30-34], is that the role of peer selection processes appears to be at least as significant as the role of peer influence processes, certainly during adolescence. 


\section{PITFALLS AND GAPS OF PREVIOUS RESEARCH}

After decades of research attempting to disentangle selection and influence processes, some important limitations remain present and will be discussed in detail in this section. The pitfalls of these studies are sometimes the result of limitations of traditional analysis techniques, such as regression techniques and SEM techniques. These statistical aspects will also be discussed in the next section.

\section{Projection bias}

In examining selection and influence processes within friendships, adolescents' reports on their friends' smoking behavior or on the number of friends who smoke are often used as measures of friends' smoking behavior [26, 28, 29]. This can result in smoking behavior among friends seeming more similar than it actually is, as adolescents project their own smoking behavior on their friends [35-37]. It is important to avoid projection bias by using the smoking behavior as reported by the friends themselves.

\section{The association between smoking behavior of friends and adolescents}

In many longitudinal studies, the association between friends' smoking behavior and the smoking behavior of adolescents at a later time is interpreted as social influence $[28,29]$. However, adolescents go through many friendship changes over time [38, 39]. Friends at a first measurement might no longer be friends at follow-up. If such friendship changes are not accounted for, this method can lead to biased estimations of social influence.

\section{The impact of friendship reciprocity}

Previous research has demonstrated that the social position of an adolescent within peer group structures is associated with differences in smoking behavior, selection, and influence processes [27, 40-43]. Several studies for example suggested differences between reciprocal and non-reciprocal friendships [27, 43]. Reciprocal friendships may offer higher friendship quality, resulting in more opportunities for influence processes to cause similarity of smoking behavior [27, 43]. Urberg also identified best friends rather than social crowd to be the major source of influence [42]. Pearson and colleagues considered differences in reciprocity and furthermore categorized all adolescents that shared reciprocal friendships into relative isolates, 
group peripherals or group members. Adolescents on the periphery of peer groups were the most important targets for selection and influence [40].

\section{The impact of developmental changes on influence and selection}

During adolescence, many developmental changes occur that might have an impact on selection and influence processes based on similar smoking behavior. Previous research demonstrated that with age, children become more and more selective. They increasingly restrict their nominations to children that return their friendships [44] and form more stable friendships [45], which implies that children become more selective in their choice of friends. Older children's criteria for choosing friends become more relevant for later phases of friendship, for friendship satisfaction and durability [46]. Therefore it is feasible that the importance of selection of friends based on similar smoking behavior may decrease with age, as similar smoking behavior will probably not be regarded as a selection criterion anymore. Age-related increase in selectivity may also be due to weakening environmental and parental constraints on youngsters' social contacts [46]. Adolescents become more independent and gain more access to potential friends, which may imply that they can afford to be selective. Another explanation for increased selectivity is based on the maturation of social cognitive skills, which may lead to using more selection criteria or gather more easily evaluative information about external and internal attributes of potential friends [46]. It is assumed that older children have more elaborated self-schemas, which enables them to look for friends whose attributes overlap with or complement their own [47]. With age, adolescents furthermore gain a longer perspective on friendships. They start evaluating what each partner gives and receives [46]. A friend's deeds are considered as an expression of the friend's personality, each trusts the other's long-term commitment to the friendship [48]. Besides changes in smoking-based selection processes, Influence processes may also change over time, as older children's disagreements become less disruptive and do not necessarily lead to negative evaluations of the self or the other anymore $[49,50]$. So it is feasible that Influence processes among friends in the context of smoking behavior may decrease with age.

\section{Differences between adolescent males and females}

Children tend to associate mainly with others of the same sex, and to have close friends almost always of the same sex [51]. Although other-sex friendships begin to develop during adolescence, sex similarity among friends is still marked [52, 53]. 
Same-sex peer relationships are an important socializing context that may result in different social-interaction styles between adolescent males and females. Girls are known to have a larger number of intimate friendships compared to boys [51, 54-56], to report a higher intimacy level compared to boys $[55,56]$, and are more likely to turn to their peers for support [57]. Furthermore, girls are more sensitive to potential costs of conflicts within a friendship and more often compromise to resolve a conflict [58]. The results of these studies may suggest that female networks offer more opportunities for influence processes to play a role. It is important to study differences between such female friendships and male friendships regarding smoking-based selection and influence processes during adolescence.

The studies presented in this dissertation will only use smoking behavior as reported by the friends themselves and will control for friendship changes and reciprocity by using structural equation modeling techniques (chapters 2 and 3) or social network analyses (chapter 4, 5, and 6). Selection and influence processes will be examined over time to account for developmental changes during adolescence and differences between male friendship networks and female friendship networks will be explored.

\section{PITFALLS OF CONVENTIONAL STATISTICAL ANALYSES TECHNIQUES}

Of the various statistical techniques used by previous researchers to disentangle selection and influence effects, until recently structural equation modeling (SEM) remained the best option as it had the least shortcomings. This statistical technique is able to examine selection and influence processes simultaneously, while mutually controlling for each process. However, this method still is limited by not being able to address three main issues that are fundamental for the separation of selection and influence processes: (1) modeling the occurrence of changes in friendships and smoking behavior between consecutive observation moments in a more realistic way (2) controlling for alternative selection mechanisms, and (3) allowing network dependencies to occur [59].

First, longitudinal data are mostly gathered at only a few discrete moments in time (panel data). As the processes of network evolution and behavior change were not traced in continuous time, it is impossible to unequivocally identify which process is responsible for network or behavioral change. For many individuals the set of friends 
at one observation moment will be different from the set of friends at the next observation moment, and in SEM it is necessary to make decisions, with arbitrary components, to specify whose influence has played a role in such cases. But even if the set of friends remained the same, the following sketch illustrates that there still is ambiguity left. In between two discrete observations, a change might have occurred as well as a change back to the original value during the same period. Figure 2 demonstrates a diagnosed influence process that was actually selection based on similar smoking behavior when observing all changes made in continuous time. The longer the time interval, the higher the possibility alternative trajectories take place. By using SEM techniques, such changes in-between two discrete observations are not modeled and the occurrence of influence will be wrongfully assumed in this case.

Figure 2 Incomplete observations of change

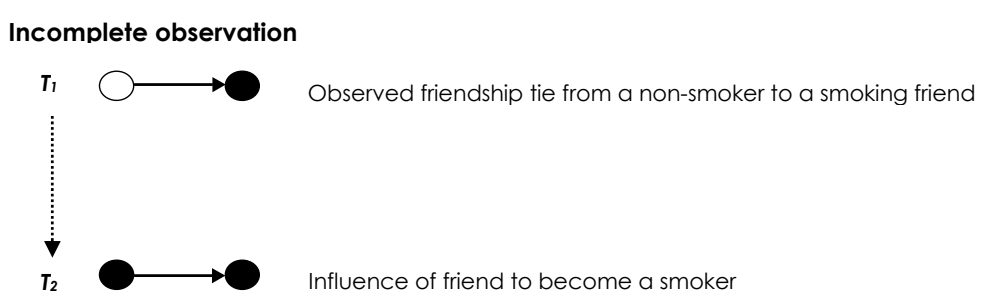

\section{Complete observation}

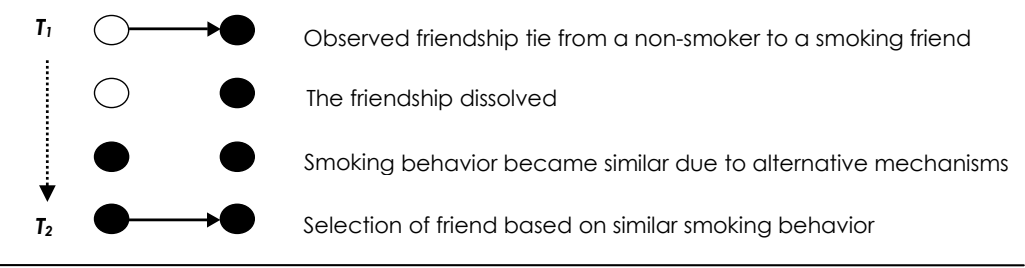

$O=$ non-smoker; $\quad$ = smoker; $\longrightarrow=$ friendship tie; $T_{1}=$ observation $1 ; T_{2}=$ observation 2.

Second, even when data would be completely observed, the problem of alternative mechanisms remains in SEM analyses. The selection of friends takes place not only based on attributes such as smoking behavior but also on the basis of current network structure. One example is 'transitivity', which is one of several purely structural network mechanisms that are known to play a strong role in friendship formation. Transitivity reflects the tendency to select those friends that are already friends of your friends regardless of their smoking behavior $[60,61]$. Not controlling for 
such mechanisms would mistakenly lead to diagnosing selection based on similar smoking behavior.

Third, when analyzing data of a complete network, the smoking behavior score of a specific individual can appear in more than one observation in the dataset. Smoking behavior of many individuals will also appear as smoking behavior scores of friends in observations of other individuals. This phenomenon violates independence of observations, a crucial assumption of conventional statistical methods, including SEM analyses.

Recently developed social network analyses techniques, however, account for these three shortcomings described above. During the course of this dissertation project, a new social network analysis method was developed and implemented in the Simulation Investigation for Empirical Network Analyses (SIENA) software, capable of assuming that changes may occur continuously in between different observation moments, taking alternative explaining selection mechanisms such as transitivity into account, and handling network dependencies [62]. In the next sections, this new method to model network and behavior co-evolution, incorporated in the SIENA software, will be explained. In chapter seven of the present dissertation, advantages and disadvantages of both analyses techniques will be further discussed.

\section{SOCIAL NETWORK ANALYSES}

\section{Longitudinal social network models}

Starting in the 1970s network analysts proposed to account for continuous changes in networks between discrete observations, by using continuous-time Markov chains [63]. In continuous-time Markov chains developmental trajectories of an individual's network are represented as a process taking place continuously over time and each of the network changes made by an individual are assumed only to depend on the current state of the network and not on the past. The current state of the network captures all the information that could influence future evolution of the network. Future states of the network will be reached through a probabilistic instead of a deterministic process. At each step the current state may be changed to another state (for example the transition from being without friends to choosing one friend or remain in the same state (staying without friends), according to a probability distribution. Such a stochastic model can be fitted to data collected at discrete 
observation moments, e.g. by imputing likely trajectories between observation moments.

The reciprocity model of Wasserman [63] is an example of a continuous-time Markov chain model of social networks which furthermore was able to account for selection of reciprocal and non-reciprocal individuals. This reciprocity model was extended by Snijders $[64,65]$ to account for other more complex alternative selection mechanisms such as transitivity (being friends of your friends' friends). However, not until recently, this model [63] has been extended to include behavior evolution as well as network evolution. In the next paragraph this model, which is called 'the actor-based model for network-behavior co-evolution' will be explained in detail, in the context of friendship networks and adolescent smoking behavior.

\section{An actor-based model for co-evolving friendship networks and smoking behavior}

Actor-based models for network-behavior co-evolution assume that at two or more observation moments, a directed network and one or more behavioral variables are observed for a finite set of social actors. The network is represented by a dichotomous relational variable, indicating for example who directs friendship ties to whom. The behavior, like for example smoking behavior, is assumed to be a dichotomous or discrete ordinal variable. Adolescents can change their smoking behavior, and also their friendship ties, in response to the current friendship network structure and the smoking behavior of the other adolescents in the school network. Each adolescent is thought to make decisions to change their friendship ties or smoking behavior to optimize his or her position in the network according to shortterm tendencies and constraints as well as a residual unknown element, modeled as a random deviation. These short-term tendencies and constraints are modeled in the actor-based model for co-evolving friendship networks and smoking behavior. An adolescent may for example choose to make a change in his or her friendship network and choose a particular friend based on his or her tendency to choose smokers as friends. Another adolescent may choose to smoke at a lower rate based on the smoking behavior of his or her parents, which may act as a constraint when they are non-smokers. Two modeling parts can be distinguished in the actor-based model: one part models friendship network changes and the other part models smoking behavior changes. These two models are integrated and tested simultaneously to account for the interdependency between friendship networks and smoking behavior. In this manner, the current state of the continuously changing friendship network can be a stimulus and constraint for changes in smoking behavior, 
while at the same time the current state of smoking behavior can act as a stimulus and constraint for changes in the friendship network.

Three statistical assumptions are made to simplify the modeling procedures: First, changes between measurement points are modeled as continuous-time Markov chains. This means that for obtaining parameter estimates, the model imputes likely behavioral and network change trajectories between two observation moments, and that the changes adolescents make in their friendship network or smoking behavior are assumed to depend only on the current state of affairs. Second, actors may change only one friendship tie or one level of smoking behavior at any moment in time. Simultaneous changes of two friendship ties for example are modeled as two successive changes in time. A change in smoking behavior from score 2 to score 4 is modeled by a change from score 2 to 3 followed by a change from score 3 to 4 . Third, actors react to each others' changes in friendship ties and smoking behavior, but do not negotiate or otherwise make joint changes based on prior agreement. An agreement like 'when you start smoking, l'll become your friend' is modeled as resulting from two smaller changes. Between those changes, the causal link cannot be enforced: 'you may start smoking, but whether that will result in a friendship remains to be seen' [65].

The actor-based model for co-evolving friendship networks and smoking behavior is used to disentangle selection and influence processes in chapters 4, 5, and 6 of the present dissertation. Since the actor-based model can include different selection and influence effects, a detailed description of the actor-based model is given in each of these chapters.

\section{PROJECT OVERVIEW}

Our main interest in this research is to disentangle the role of selection and influence processes among adolescent friends in the context of smoking behavior.

\section{Research Questions}

1. Do friends select each other based on similar smoking behavior?

2. Do friends influence each other in smoking behavior?

3. What is the role of friendship reciprocity in influence and selection processes?

4. Do influence and selection processes change over time?

5. What is the role of gender? 


\section{Characteristics of the project}

These research questions are treated here in five studies, presented in chapters 2-6. The main characteristics of the five studies are presented in Table 1. All studies use data collected by De Vries and colleagues [66] during the European Smoking prevention Framework Approach (ESFA) study in six European countries: Finland, Denmark, the Netherlands, Portugal, Spain, and the United Kingdom. Data were collected for the first time in autumn 1998 among $7^{\text {th }}$ graders. Follow up was conducted 12,24, and 30 months later. All pupils present in the schools on the days of data collection completed the questionnaire and it was explained to them that responses would be treated confidentially and that they could refuse to participate. This dissertation includes only the adolescents that participated in the control group. Findings regarding the Dutch sample are presented in chapters 2 and 3. Chapter 4 presents the findings of the Dutch, Danish, Finnish, English, Spanish, and Portuguese samples, while Chapter 5 and 6 present findings for the Finnish sample.

Table 1 Characteristics of the five studies described in the current dissertation

\begin{tabular}{|c|c|c|c|c|c|}
\hline & Study 1 & Study 2 & Study 3 & Study 4 & Study 5 \\
\hline Chapters & 2 & 3 & 4 & 5 & 6 \\
\hline Research question & $1,2,3$ & $1,2,3,4$ & $4,5,6$ & $3,4,5,6$ & $3,4,5,6,7$ \\
\hline \multirow[t]{5}{*}{ Country } & The & The & The & Finland & Finland \\
\hline & Netherlands & Netherlands & Netherlands, & & \\
\hline & & & Denmark, & & \\
\hline & & & Finland, Spain, & & \\
\hline & & & Portugal, UK & & \\
\hline Data & 1998 and & 1998,1999 & 1998, 1999, & 1998, 1999, & 1998, 1999, \\
\hline \multirow[t]{2}{*}{ collection } & 1999 & 2000 , and 2001 & 2000 , and 2001 & 2000 , and & 2000 , and 2001 \\
\hline & & & & 2001 & \\
\hline Sample size & 1886 & 1886 & 7704 & 1326 & 1163 \\
\hline
\end{tabular}

\section{Outline of the dissertation}

In the first part of this dissertation we use structural equation modeling techniques to examine selection and influence processes among adolescent friends (chapters 2 and 3), whereas in the second part of this dissertation we use social network analysis techniques (Chapters 4-6).

In chapter 2 we examine social selection and social influence within adolescent friendships, and the role of parents and siblings, as factors explaining similarity of smoking behavior among adolescent friends. This chapter addresses 
three often neglected design issues to improve our understanding of the role of selection and influence processes. We use smoking behavior as reported by the friends themselves instead of adolescents' reports on their friends' smoking behavior. We examine social selection and social influence simultaneously while considering friendship composition changes, and focus specifically on both reciprocal and nonreciprocal friends.

In chapter 3 using longitudinal analyses we explore the contribution of selection and influence processes in smoking behavior similarity among friends, and specifically focus on changes in these processes during early and mid adolescence. We again use the smoking behavior of friends as reported by themselves and consider friendship composition changes and reciprocity. As it is possible that friendships are terminated based on smoking behavior dissimilarities among friends, we furthermore examine the effects of smoking behavior of former friends.

In chapter 4 the co-evolution of friendship networks and adolescent smoking behavior is examined in 6 European countries. Selection and influence processes are disentangled by using new methods of social network analysis that are capable of assuming that changes may occur continuously in between different observation moments, taking alternative explaining selection mechanisms such as transitivity into account, and handling network dependencies.

In chapter 5 the mutual influence of friendship networks and smoking behavior is studied in-depth among Finnish adolescents. In this chapter, newly developed social network analyses techniques are used. We examine whether the strength of smoking-based selection and influence processes differ between reciprocal and non-reciprocal friendships.

In chapter 6 we examine differences between male friendship networks and female friendship networks among Finnish adolescents. Smoking-based selection and influence processes are examined in these gender segregated friendship networks, again by using social network analysis techniques.

Finally, in chapter 7 the main findings of the 5 studies are summarized and discussed. We address theoretical and practical implications and directions for future research. 



\section{CHAPTER 2}

Disentangling selection and influence effects on adolescent smoking

Based on: Mercken, L., Candel, M., Willems, P., \& de Vries, H. (2007) Disentangling social selection and social influence effects on adolescent smoking: the importance of reciprocity in friendships. Addiction, 102 (9), 1483-1492. 


\section{ABSTRACT}

Aims: The goal of this study was to examine social selection and social influence within reciprocal and non-reciprocal friendships, and the role of parents and siblings, as factors explaining similarity of smoking behavior among adolescent friends. A new social selection-social influence model is proposed. Design: Longitudinal design with two measurements. Setting: Data were gathered among Dutch high school students in the control group of the European Smoking prevention Framework Approach (ESFA) study. Participants: The sample consisted of 1886 adolescents with a mean age of 12.7 years. Measurements: The main outcome measures were the smoking behaviors of the respondents, best friends, parents, and siblings. We tested the social selection-social influence model with structural equation modeling techniques. Findings: Social selection and social influence both played an important role in explaining similarity of smoking behavior among friends. Within non-reciprocal friendships, only social selection explained similarity of smoking behavior, whereas within reciprocal friendships, social influence and possibly also social selection explained similarity of smoking behavior. Sibling smoking behavior was a more important predictor of adolescent smoking behavior than parental smoking behavior. Conclusions: Social selection and social influence both promote similarity of smoking behavior, and the impact of each process differs with the degree of reciprocity of friendships. These insights may contribute to further refinement of smoking prevention strategies. 


\section{INTRODUCTION}

Smokers befriend smokers and non-smokers befriend non-smokers $[9,10]$. Peer groups play a crucial role in adolescent smoking behavior [8-11]. Smoking behavior is often similar among friends as a result of social selection and/or social influence. The influence hypothesis posits that friendships cause smoking behavior, whereas the selection hypothesis posits that smoking behavior promotes friendships.

Friends can have similar smoking behaviors when friendships are formed on the basis of a common smoking behavior. Smokers select smoking friends and nonsmokers select non-smoking friends. In these cases, social selection based on common smoking behavior produces similarities in smoking behavior among friends $[12,13,35]$. Social influence among friends occurs when smoking behavior within established friendships becomes similar. Adolescents start smoking in response to direct pressure by, or the example of, peer friends [12, 13]. Smoking behavior within friendships can also become similar when friends do not influence each other, but are exposed to a situation or person of influence [67]. Children are more likely to smoke if one or both parents smoke [68-71] and there is less transfer of knowledge, attitudes, and skills that prepare children to resist smoking [72]. The few studies that have examined the effects of sibling smoking also show an association between sibling smoking and adolescent smoking [18].

Currently, most smoking prevention programs are based on the assumption that smoking onset is caused by peer smoking and by an incapability among adolescents to resist peer pressure $[73,74]$. Nonetheless, mounting evidence suggests that social selection may be just as important as, or even more important than, social influence in explaining similarity of smoking behavior within friendships $[12,13,23,26$, $29,36,75]$. In this context, it is important to acknowledge three often-neglected design issues to improve our understanding of the role of selection and influence processes.

First, in examining social selection and social influence within friendships, adolescents' reports on their friends' smoking behavior or on the number of friends who smoke are often used as measures of friends' smoking behavior [26, 28, 29]. This can result in smoking behavior among friends seeming more similar than it actually is, as adolescents project their own smoking behavior onto their friends [35-37]. It is important to avoid projection bias by using the smoking behavior as reported by the friends themselves. 
Second, in longitudinal studies, an association between friends' smoking behavior and the smoking behavior of adolescents at a later time is often interpreted as social influence [28, 29]. However, adolescents go through many friendship changes over time $[38,39]$. Friends at a first measurement might no longer be friends at follow-up. If such friendship changes are not considered, this method can lead to biased estimations of social influence.

Third, previous research has indicated that the social position of an adolescent within peer group structures is associated with differences in smoking behavior, selection, and influence processes [27, 40-43]. Several studies suggested differences between reciprocal and non-reciprocal friendships [27, 43]. Reciprocal friendships offer higher friendship quality, resulting in more opportunities for influence processes to cause similarity of smoking behavior [27, 43]. Urberg also identified best friends rather than social crowd to be the major source of influence [42]. Pearson and colleagues considered differences in reciprocity and furthermore categorized all adolescents who shared reciprocal friendships into relative isolates, group peripherals or group members. Adolescents on the periphery of peer groups were the most important targets for selection and influence [40]. Therefore, it is important to consider reciprocity when studying selection and influence processes.

The goal of this study is to examine social selection and social influence of friends, parents, and siblings, taking all the issues mentioned into account. We examine selection and influence processes simultaneously, and focus specifically on both reciprocal and non-reciprocal friends.

\section{METHODS}

\section{Participants}

The sample consisted of 1886 adolescents in nine randomly selected Dutch schools that participated as a control group in the European Smoking prevention Framework Approach (ESFA) study [66]. Adolescents participating in the experimental group were not included, as the smoking prevention intervention was effective in the Netherlands [76], and relationships between variables of interest might also have changed. 


\section{Procedure}

We distributed self-administered questionnaires among all $7^{\text {th }}$ graders of each participating school during the autumn of 1998. Follow-up was conducted 12 months later [66, 76]. We asked all students present on the day of data collection to complete the questionnaire and explained to them that responses would be treated confidentially and that they could refuse to participate. Students returned their questionnaires in sealed envelopes to guarantee their anonymity. At baseline, the proportion of refusals to participate was $0.56 \%$ in the Netherlands [66].

\section{Questionnaire}

The ESFA questionnaire was based on earlier studies about adolescent smoking behavior [76-80].

The respondents' smoking behavior was assessed with one question: 'On average, how many cigarettes do you smoke during a week (including the weekend) ?' $(0=0,1=$ between 0 and $1,2=2-10,3=11-30,4 \geq 30)$.

Best friends' smoking behavior was assessed primarily by means of a name generator in which each respondent could name up to five 'best friends' inside and/or outside school [81]. Of these friends, only best friends inside school in the same grade were included, as only they also filled in the questionnaire at exactly the same time. Friends outside or inside the school, but not in the same grade as the respondents who nominated them, were excluded. The self-reported smoking behavior of each friend was traced back in their personal questionnaire. In view of the ordinal nature of the respondent's smoking behavior variable, we calculated a median score of the smoking behavior of each respondent's best friends.

Parental smoking behavior was measured by two questions: 'Does your father (or male caregiver) smoke?' and 'Does your mother (or female caregiver) smoke?'. The answer was recoded into one variable $10=$ neither of my parents smoke, $1=$ at least one of my parents smokes).

Sibling smoking behavior was measured by two questions: 'Do one or more of your brother(s) smoke?' and 'Do one or more of your sister(s) smoke?', and was recoded into one variable $(0=$ neither of my siblings smoke, $1=$ at least one of my siblings smokes).

We also recorded age, gender $(0=$ boy, $1=$ girl $)$, ethnicity $(0=$ nonnative, $1=$ native), religion ( $0=$ not religious, $1=$ religious $)$, and alcohol consumption $(0=0$ glasses of alcoholic drinks per week, $1=1$ or 2 glasses, $2=3$ to 5 glasses, $3=$ more 
than 5 glasses; 1 glass contains approximately 10 grams of pure alcohol), which served as covariates in the analysis.

\section{Model development}

To examine social selection and influence, we developed an explorative model. First, we took friendship changes into account by identifying friends indicated at the second measurement $\left(T_{2}\right)$ as either newly chosen or long-standing friends. Newly chosen friends had been selected for the first time by a respondent at $T_{2}$, whereas long-standing friends had already been selected at the first measurement $\left(T_{1}\right)$ and were still friends at $T_{2}$. Figure 1 illustrates the resulting model, in which selection is reflected by the way respondents select friends, and influence by the way respondents influence friends.

Figure 1 Basic model including newly chosen and long-standing best friends

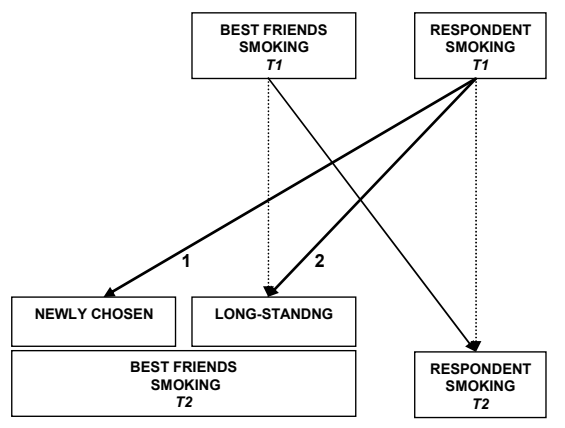

$T_{1}=$ first measurement; $T_{2}=$ second measurement; Bold lines = selection and influence paths; Dotted lines $=$ influence of own previous behavior

The association between the smoking behavior of the respondents at $T_{1}$ and longstanding friends at $T_{2}$ (path 2) represents social influence only. By excluding dissolved friendships, we make this path an unbiased estimation of social influence of respondents on their friends. The association between the smoking behavior of respondents at $T_{1}$ and newly chosen friends at $T_{2}$ (path 1) reflects social selection. Path 1, however, could also represent social influence, as the respondent could have influenced newly selected friends immediately after selection took place. To examine social selection, we have to distinguish between reciprocal and non-reciprocal friends. Nominated reciprocal friends perceive the adolescent to be their friend as well, whereas nominated non-reciprocal friends do not perceive the adolescent to be their friend. Immediately after selection of non-reciprocal friends, respondents 
probably cannot influence these friends, since non-reciprocal friends do not perceive the respondent as a friend. In this case, only selection can explain smoking behavior similarity.

The final model that makes the distinction clearly between non-reciprocal and reciprocal friends and also includes parental and sibling smoking behavior is shown in Figure 2. Paths 1 and 3 represent social selection. The association between adolescent smoking behavior at $T_{1}$ and non-reciprocal newly chosen friends' smoking behavior at $T_{2}$ (path 1) very probably reflects only social selection, whereas path 3 can reflect social influence after selection took place as it involves reciprocal friends. Paths 2 and 4 represent social influence within non-reciprocal and reciprocal friendships, respectively. Selection processes, of course, might have been operating before $T_{1}$ to produce these long-standing friendships. The associations between the smoking behavior of reciprocal and non-reciprocal friends at $T_{1}$, and the respondents at $T_{2}$ also represent a social influence of friends. However, because we considered all the respondent's friends at $T_{1}$, including those who were no longer the respondent's friends at $T_{2}$, these associations probably underestimate social influence. Paths 5,6 , and 7 represent the social influence of parents and siblings. Dotted paths represent future behavior being correlated with the respondent's own previous behavior. The model included covariates of adolescent smoking behavior, so that age, gender, ethnicity, religion and alcohol consumption were accounted for. The covariates were allowed to influence the smoking behavior of all actors, both at $T_{1}$ and $T_{2}$.

Figure 2 Hypothesized social selection-social influence model

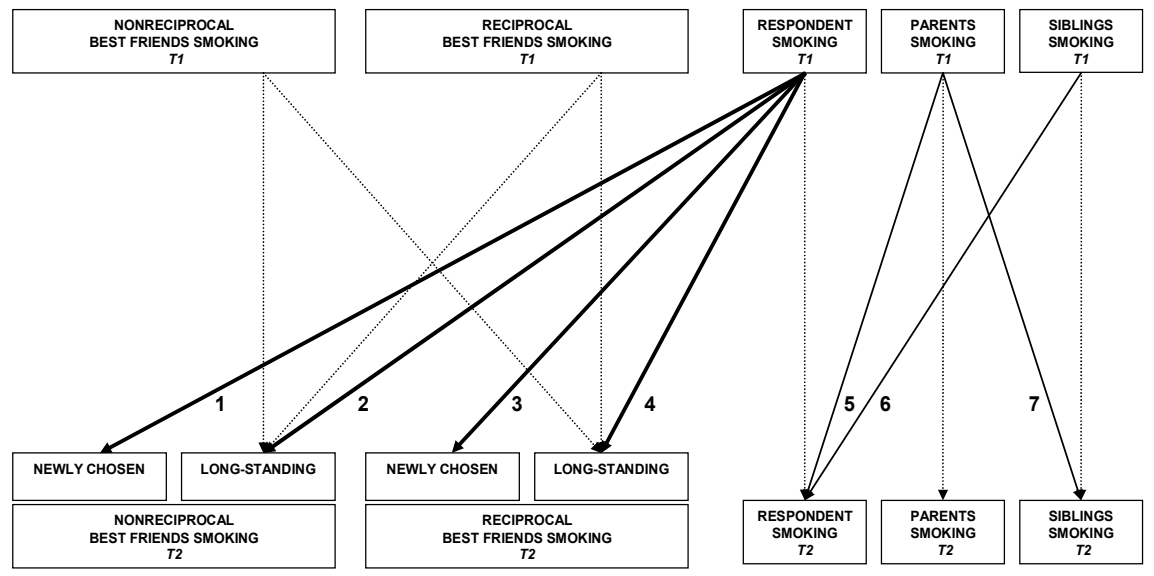

$T_{1}=$ first measurement; $T_{2}=$ second measurement; Bold lines = selection and influence paths; Non-dotted, non-bold lines = influence of parents and siblings; Dotted lines = influence of past behavior 


\section{Statistical analyses}

We tested the model using structural equation modeling (SEM), with the help of Mplus 4.1 software [82]. As the variables turned out to be not normally distributed, a robust maximum likelihood (MLR) analysis was used [83]. MLR produces maximum likelihood parameter estimates and standard errors that are robust to non-normality. The MLR standard errors are computed with a sandwich (robust covariance matrix) estimator [84]. MLR is based on all available data (not only complete cases) and allows data to be missing at random (MAR) [82].

For measures of overall model fit, we present the Comparative Fit Index (CFI) and the Tucker-Lewis Index (TLI). A good model fit is indicated by CFI and TLI values higher than 0.90 [85]. In addition, as the sample size was large enough, we report the root mean square error of approximation (RMSEA). The RMSEA is an estimation of the lack of fit in a model compared to a perfectly saturated model. RMSEA values lower than 0.05 indicate a good model fit [85].

\section{RESULTS}

\section{Descriptives}

The sample consisted of 1886 Dutch adolescents who completed the questionnaire at $T_{1}$. At $T_{2}, 1763$ completed the questionnaire (93.5\% of $\left.T_{1}\right)$. Logistic regression analysis showed that response at $T_{2}$ was predicted significantly by the respondents' alcohol consumption $(\mathrm{OR}=1.008 ; 95 \% \mathrm{Cl}=1.002-1.014 ; p<0.01)$ and friends' smoking behavior $(O R=1.605 ; 95 \% \mathrm{Cl}=1.156-2.228 ; p<0.01)$. Respondents who drank less alcohol and respondents with best friends who smoked less were significantly more likely to respond at $T_{2}$.

Of all 1886 respondents, $50.1 \%$ were girls, $76.1 \%$ were of Dutch origin, and $49.8 \%$ said they had some religious background. The mean age of the respondents was 12.7 years $(S D=0.61)$. The respondents nominated 5705 best friends at baseline and 6695 at $T_{2}$. Of these, 2967 friends at baseline and 3716 friends at $T_{2}$ were in the same school and grade as the respondents who nominated them. At baseline, $56 \%$ of the best friends were non-reciprocal and $44 \%$ were reciprocal. At $T_{2}, 40 \%$ were non-reciprocal newly chosen best friends; $10 \%$, non-reciprocal long-standing; $34 \%$, reciprocal newly chosen; and $16 \%$, reciprocal long-standing. The number of reciprocal friendships increased over time. Table 1 depicts the smoking behavior of respondents, best friends, parents, and siblings, and alcohol consumption of 
CHAPTER 2 | DISENTANGLING SELECTION AND INFLUENCE

respondents at baseline. Table 2 shows the correlation matrix for the smoking behavior of respondents, best friends, parents, and siblings at $T_{1}$ and $T_{2}$.

Table1 Baseline characteristics

\begin{tabular}{llllllll}
\hline & \multicolumn{7}{l}{ Smoking behavior } \\
\cline { 2 - 7 } & $\mathbf{0}$ & $\mathbf{1}$ & $\mathbf{2}$ & $\mathbf{3}$ & $\mathbf{4}$ & Missing \\
\hline Adolescents* & 91.5 & 3.9 & 2.6 & 1.0 & 0.7 & 0.3 \\
Nominated best friends* & 58.8 & 3.0 & 1.4 & 0.5 & 0.2 & $36.1^{* * *}$ \\
Parents** & 48.2 & 51.6 & - & - & - & 0.2 \\
Siblings** & 85.8 & 14.1 & - & - & - & 0.2 \\
\hline & Alcohol consumption^ & & & \\
\cline { 2 - 8 } & $\mathbf{0}$ & $\mathbf{1}$ & $\mathbf{2}$ & $\mathbf{3}$ & - & Missing \\
\hline Adolescents & 77.9 & 4.1 & 1.0 & 0.7 & - & 16.3 \\
\hline
\end{tabular}

*Smoking behavior adolescents and median smoking behavior of nominated best friends: $0=0$ cigarettes during a week; $1=$ between 0 and $1 ; 2=2-10 ; 3=11-30 ; 4=>30 ;{ }^{*}$ Smoking behavior parents and siblings: $0=$ none of them smokes; $1=$ at least one of them smokes; ${ }^{* *} 21.8 \%$ of the adolescents nominated no best friends; $14.3 \%$ nominated only adolescents outside school or from other grades; $\wedge$ Alcohol consumption: $0=$ 0 glasses of alcoholic drinks per week; $1=1-2 ; 2=3-5 ; 3=>5$. 


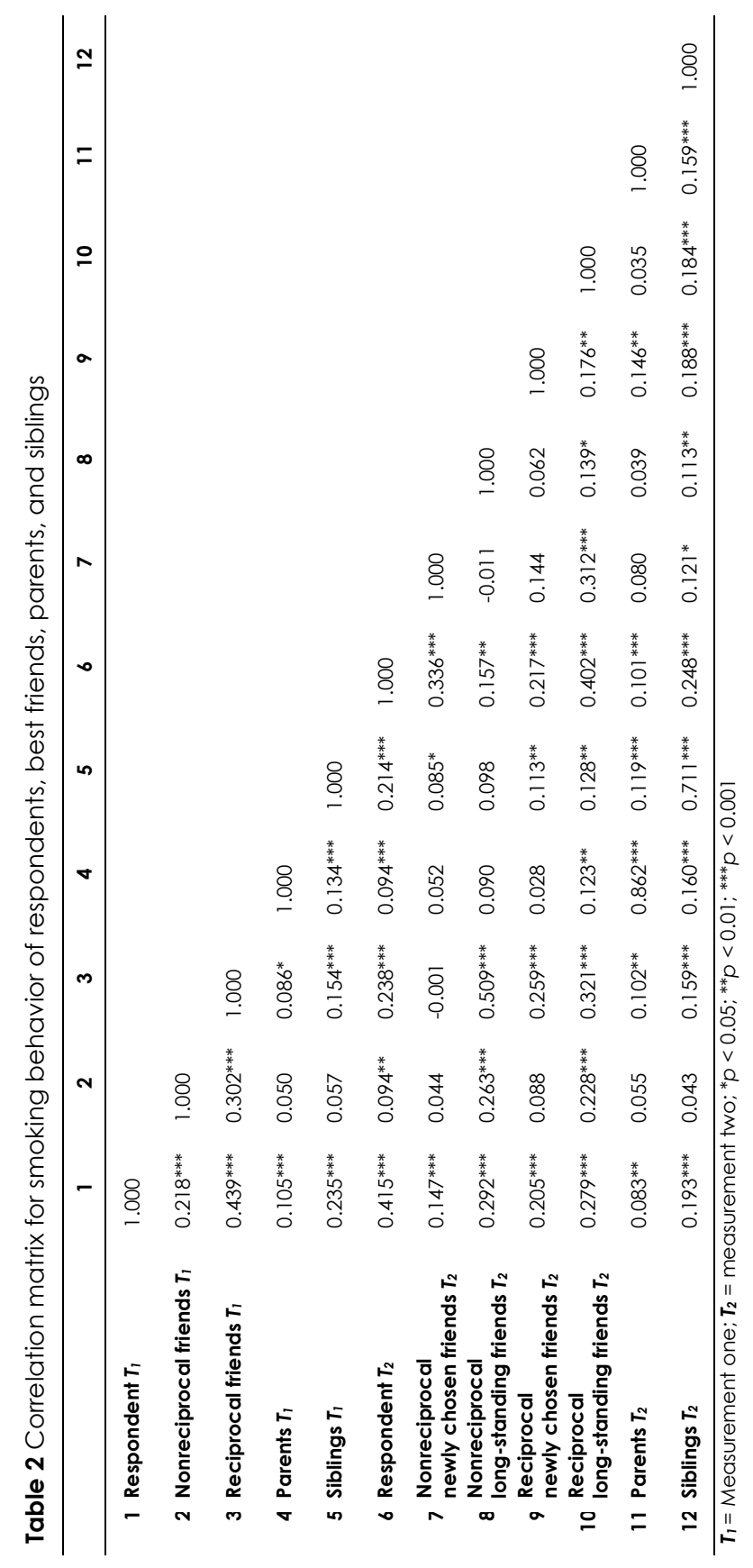




\section{The social selection-social influence model}

Figure 3 depicts the results of the social selection-social influence model. All rectangles represent measured variables. Absent or dotted lines connecting variables imply that significant effects were not found. The model fits the data very well $(\mathrm{CFI}=1.000 ; \mathrm{TLI}=1.015$; RMSEA $=0.000)$. All path coefficients of the model, including non-significant paths, are listed in table 3.

Figure 3 Social selection-social influence model: resulting path coefficients and significance levels

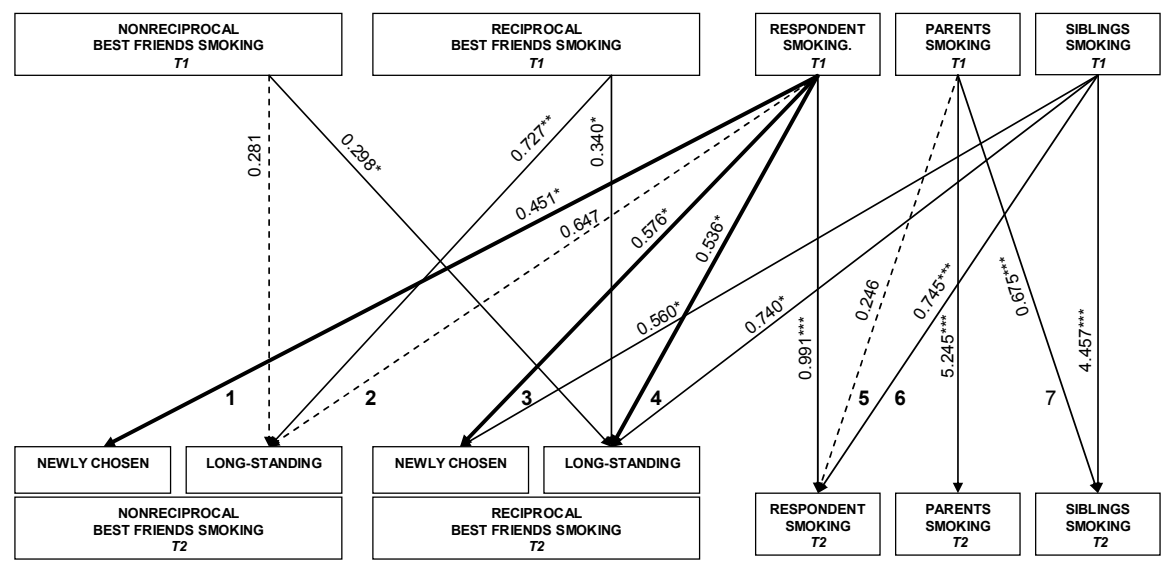

$T_{1}=$ first measurement; $T_{2}=$ second measurement; Bold lines= hypothesized selection and influence paths; Non-dotted, non-bold lines = all other significant paths; Dotted lines= influence of past behavior; ${ }^{*} p<0.05,{ }^{* *} p<0.01,{ }^{* * *} p<0.001$

\section{Non-reciprocal friendships}

The association between the smoking behavior of respondents at $T_{1}$ and nonreciprocal newly chosen friends at $T_{2}$ (path 1) was positive and significant $(p<0.05)$, indicating that respondents selected non-reciprocal friends with similar smoking behavior. The association between the smoking behavior of respondents at $T_{1}$ and non-reciprocal long-standing friends at $T_{2}$ (path 2) was not significant ( $p>0.05$ ). This confirms that the respondents did not influence their non-reciprocal friends and suggests furthermore that path 1 represents solely social selection. 


\section{Reciprocal friendships}

The association between the smoking behavior of respondents at $T_{1}$ and reciprocal newly chosen friends at $T_{2}$ (path 3 ) was positive and significant $(p<0.05)$, indicating that respondents selected reciprocal friends with similar smoking behavior. The significant path coefficient of $0.536(p<0.05)$ from the smoking behavior of respondents at $T_{1}$ to that of reciprocal long-standing friends at $T_{2}$ (path 4 ) indicates the contribution of social influence to the similarity of smoking behavior between the respondents and their reciprocal friends.

\section{Parents and siblings}

The effect of parental smoking behavior at $T_{1}$ on respondents' smoking behavior at $T_{2}$ (path 5), and the path coefficients from parental smoking behavior at $T_{1}$ to that of all four categories of friends, were not significant $(p>0.05)$. The association between parental smoking behavior at $T_{1}$ and sibling smoking behavior at $T_{2}$ (path 7) was significant $(p<0.001)$.

The effect of sibling smoking behavior at $T_{1}$ on the respondent's smoking behavior at $T_{2}$ (path 6) was significant $(p<0.001$ ). The paths from sibling smoking behavior at $T_{1}$ to that of reciprocal newly chosen friends and reciprocal longstanding friends were both significant ( $p<0.001$ ), indicating that siblings also influenced the reciprocal friends of their brother or sister.

\section{Covariates}

Of the covariates assessed, alcohol consumption and gender were associated with the respondents' smoking behavior at $T_{2}$. Respondents who drank more alcohol smoked more cigarettes (coefficient $=0.608 ; p<0.001$ ). Girls smoked significantly more cigarettes than boys did (coefficient $=0.356$; $p<0.05$ ). 
Table 3 Differences between reciprocal and non-reciprocal friendships: results of the structural equation modeling using robust maximum likelihood

\begin{tabular}{|c|c|c|c|c|}
\hline Estimated Path & Path coefficient & s.e. & $t$ & $p$ \\
\hline Non-reciprocal friends - non-reciprocal newly chosen friends & 0.031 & 0.127 & 0.245 & 0.806 \\
\hline Non-reciprocal friends - non-reciprocal long-standing friends & 0.281 & 0.147 & 1.917 & 0.055 \\
\hline Non-reciprocal friends - reciprocal newly chosen friends & 0.084 & 0.166 & 0.509 & 0.611 \\
\hline Non-reciprocal friends - reciprocal long-standing friends & 0.298 & 0.150 & 1.984 & 0.047 \\
\hline Non-reciprocal friends - respondent & 0.082 & 0.113 & 0.729 & 0.466 \\
\hline Non-reciprocal friends - siblings & -0.024 & 0.164 & -0.144 & 0.886 \\
\hline Reciprocal friends - non-reciprocal newly chosen friends & -0.104 & 0.156 & -0.664 & 0.507 \\
\hline Reciprocal friends - non-reciprocal long-standing friends & 0.727 & 0.267 & 2.720 & 0.007 \\
\hline Reciprocal friends - reciprocal newly chosen friends & 0.245 & 0.130 & 1.876 & 0.061 \\
\hline Reciprocal friends - reciprocal long-standing friends & 0.340 & 0.173 & 1.965 & 0.049 \\
\hline Reciprocal friends - respondent & 0.125 & 0.117 & 1.068 & 0.286 \\
\hline Reciprocal friends - siblings & 0.165 & 0.133 & 1.236 & 0.216 \\
\hline Respondent - respondent & 0.991 & 0.140 & 7.094 & 0.000 \\
\hline Respondent - non-reciprocal newly chosen friends (path 1) & 0.451 & 0.176 & 2.562 & 0.010 \\
\hline Respondent - non-reciprocal long-standing friends (path 2) & 0.647 & 0.378 & 1.714 & 0.087 \\
\hline Respondent - reciprocal newly chosen friends (path 3) & 0.576 & 0.252 & 2.288 & 0.022 \\
\hline Respondent - reciprocal long-standing friends (path 4) & 0.536 & 0.267 & 2.002 & 0.045 \\
\hline Respondent - siblings & 0.165 & 0.185 & 0.891 & 0.373 \\
\hline Parents - parents & 5.245 & 0.195 & 26.962 & 0.000 \\
\hline Parents - non-reciprocal newly chosen friends & 0.153 & 0.164 & 0.931 & 0.352 \\
\hline Parents - non-reciprocal long-standing friends & 0.599 & 0.316 & 1.898 & 0.058 \\
\hline Parents - reciprocal newly chosen friends & -0.096 & 0.207 & -0.465 & 0.642 \\
\hline Parents - reciprocal long-standing friends & 0.474 & 0.265 & 1.791 & 0.073 \\
\hline Parents - respondent (path 5) & 0.246 & 0.144 & 1.702 & 0.089 \\
\hline Parents - siblings (path 7) & 0.675 & 0.190 & 3.553 & 0.000 \\
\hline Siblings - siblings & 4.457 & 0.233 & 19.090 & 0.000 \\
\hline Siblings - non-reciprocal newly chosen friends & 0.248 & 0.258 & 0.959 & 0.338 \\
\hline Siblings - non-reciprocal long-standing friends & -0.116 & 0.501 & -0.232 & 0.817 \\
\hline Siblings - reciprocal newly chosen friends & 0.560 & 0.275 & 2.040 & 0.041 \\
\hline Siblings - reciprocal long-standing friends & 0.740 & 0.356 & 2.080 & 0.038 \\
\hline Siblings - respondent (path 6) & 0.745 & 0.184 & 4.057 & 0.000 \\
\hline \multicolumn{5}{|l|}{ Log likelihood $=-17379.99$} \\
\hline \multicolumn{5}{|l|}{$\mathrm{CFI}=1.000$} \\
\hline \multicolumn{5}{|l|}{$\mathrm{TLI}=1.015$} \\
\hline RMSEA $=0.000$ & & & & \\
\hline
\end{tabular}

s.e. $=$ standard error; $t=t$ - value; $p=$ two-sided probability; CFI = comparative fit index; TLI = Tucker-Lewis index; RMSEA = root mean square error of approximation 


\section{DISCUSSION}

Our study examined social selection and social influence within reciprocal and nonreciprocal friendships, and the role of parents and siblings, as factors explaining similarity of smoking behavior among adolescent friends.

First, our results show clearly that both selection and influence processes are important in explaining the similarity of smoking behavior among friends. These findings are in agreement with those of previous research [12, 13, 23, 26-28, 75]. Ennett and Bauman created peer groups using social network techniques, and conclude that selection appears to be at least as important as influence in causing peer group homogeneity [13]. Engels and colleagues report evidence of influence and selection processes. More non-smokers with smoking friends changed their smoking behavior than did non-smokers with non-smoking friends, and adolescents selected friends with smoking behavior similar to their own [26]. A previous study with similar data report mainly evidence of selection processes [29]. However, examining friendships without distinguishing between non-reciprocal and reciprocal friends may have led to an underestimation of possible influence processes. We did not find an association between friends' smoking behavior at $T_{1}$ and the respondents' smoking behavior at $T_{2}$. This correlates with a previous study [29], but contrasts with a recent study indicating a significant association between friends' smoking behavior and adolescent smoking behavior at later times [28]. This difference may be due to the use of the friends' own reported smoking behavior, which prevented projection bias [35-37].

Second, our results clearly show differences in the effects of influence and selection processes between non-reciprocal and reciprocal friendships. Within nonreciprocal friendships, only social selection appears to cause similarity of smoking behavior, whereas within reciprocal friendships, such similarity was caused by social influence and possibly also social selection. These findings are in line with previous research [27, 43]. Urberg and colleagues examined reciprocal friendships and found evidence for selection as well as influence processes [27]. Previous studies have also suggested that reciprocal friendships offer higher friendship quality and more opportunities for interaction than non-reciprocal friendships [43]. Consequently, higher friendship quality can result in more opportunities for social influence to cause similarity of smoking behavior [27, 43].

Third, in contrast with previous studies $[29,68-71]$, we did not find a significant association between parental and respondent smoking behavior. This might be due 
to the inclusion of sibling smoking behavior in our model. In a recent review, Avenevoli and Merikangas [18] suggested that significant parental effects are generally small and are often even completely eliminated when other variables are included in the model. In-depth analyses without sibling smoking behavior indeed showed a significant positive effect of parental smoking behavior. In line with previous studies [18], sibling smoking behavior strongly influenced respondents' smoking behavior. Additionally, our findings showed that siblings also influenced the smoking behavior of their brother's or sister's reciprocal friends. Future studies need to investigate further the influence of siblings on family members and on the friends of their own siblings.

\section{Limitations}

This study was subject to some limitations. First, self-reported smoking behavior was not validated by biochemical measures. However, self-reports have been shown to be reliable and to correspond well with biological indicators when measurements are performed under optimized measurement conditions that assure anonymity [86]. During the ESFA project, measurement conditions were optimized by guaranteeing strict confidentiality of adolescent responses $[29,76,77]$. Second, no direct measures of parental smoking behavior and sibling smoking behavior were available. The effects of parental and sibling smoking behaviors may have been overestimated, although previous research has demonstrated that adolescents aged 13-17 years are reliable sources for assessing the smoking status of their parents [87]. Third, the use of a fixed-response name generator might have restricted the ability to reciprocate, as respondents were only allowed to nominate up to five best friends. However, previous research, allowing $7^{\text {th }}$ graders to nominate any number of friends, showed that on average only 4.09 friends were nominated [88]. Allowing to nominate more than five best friends, might provoke adolescents to nominate peers who are not 'best' friends. Fourth, we considered only two possible social positions by distinguishing between reciprocal and non-reciprocal friends. Previous research, using social network techniques, was able to generate more specific social positions such as relative isolates, peripherals, dyads, and groups [40,41]. A reciprocal friend can still be part of an isolated friendship pair, connected to someone within the group, but not part of the group, or a group member. Future research should also consider other social positions. Fifth, the SEM techniques do not consider all dependencies caused by network structure. Nominated friends may also be respondents in the same model, and respondents are subject not only to influences 
of multiple friends, but also to potentially important indirect "friend-of-friend" influences. New social network analysis methods that take these kinds of dependencies into account are currently being developed [89].

\section{Practical implications}

This study has several practical implications. First, smoking prevention programs should focus not only on social influence but also on selection processes. Previous research has already emphasized that the way in which peer environments are structured needs more attention within prevention programs besides the promotion of social influence skills [90-92]. Second, future research should differentiate between reciprocal and non-reciprocal friendships when investigating social selection and social influence. Our results demonstrate clearly differences in the effects of influence and selection processes between non-reciprocal and reciprocal friendships. Third, sibling smoking behavior seems to be a more important predictor of adolescent smoking behavior than parental smoking behavior. Additionally, sibling smoking behavior was associated positively with the smoking behavior of the respondents' reciprocal friends. Further research is needed to investigate the role of siblings in adolescent friendships.

\section{Conclusions}

We can draw three conclusions about the role of social selection and social influence regarding similarity of smoking behavior. First, similarity of smoking behavior between adolescent friends is caused by social selection and social influence. Second, within non-reciprocal friendships, only social selection causes similarity of smoking behavior. Within reciprocal friendships, similarity of smoking behavior is caused by social influence and possibly also by social selection. Third, sibling smoking behavior has a greater impact on smoking onset than parental smoking behavior. These insights can help to develop smoking prevention programs that focus not only on social influence, but also consider social selection as a major cause of similarity of smoking behavior among adolescent friends. 


\section{CHAPTER 3}

Changes during early and mid adolescence

Based on: Mercken, L., Candel, M., Willems, P., \& de Vries, H. (2009) Social influence and selection effects in the context of smoking behavior: Changes during early and mid adolescence. Health Psychology, 28(1), 73-82. 


\section{ABSTRACT}

Aims: This article examined the contribution of selection and influence processes in smoking behavior similarity among friends, and changes in these processes during early and mid adolescence. Design: Data of 1886 Dutch high school students in the control group of the European Smoking prevention Framework Approach (ESFA) study were used. Changes in selection and influence were examined during three successive waves using structural equation modeling. Measurements: Smoking behavior of adolescents, best friends, parents and siblings. Findings: Most support was

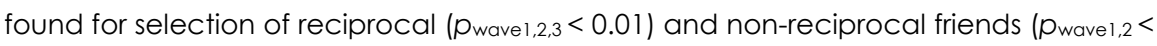
0.01 ; P wave $_{\text {w }}=0.25$ ) although these effects decreased over time. Support for influence was only found among non-reciprocal (desired) friends during the last wave (pwave3 $<$ 0.01 ). Adolescents were influenced by their parents ( $p_{\text {wave } 2}<0.01 ; p_{\text {wave }}, 3>0.05$ ) and siblings ( $p_{\text {wave } 1,2<0.01 ;}$ p wave3 $=0.16$ ), but influence diminished over time. Conclusions: Smoking-based selection processes decreased over time while the influence of friends increased. Smoking prevention programs should focus on the structure of peer environments besides promoting social influence skills. During early adolescence parents and siblings should be targeted, while during mid adolescence, the focus should shift toward the adolescents and their dynamic peer environment. 


\section{INTRODUCTION}

Tobacco is the fourth most common risk factor for disease and the second major cause of death worldwide [93]. In most Western countries, there is an increase in the prevalence of smoking during adolescence [2,3]. For 13-year olds, the prevalence of regular smoking varies from $3.5 \%$ to $12.5 \%$ and increases to $17 \%$ to $24,5 \%$ for 15 -year olds [4]. Insight into the different processes that might explain smoking initiation during adolescence may facilitate development of prevention programs and adaptation of policies regarding effective smoking prevention.

Adolescent peer groups play a crucial role in the initiation and maintenance of smoking behavior as evidenced by a substantial body of research suggesting that smokers befriend smokers and non-smokers befriend non-smokers [9]. Similarity in smoking behavior among friends may occur as a result of selection and influence processes. Similarity in smoking behavior due to selection occurs when adolescents select friends with similar smoking behavior during the formation of a friendship [12, 13]. When smokers select smoking friends and non-smokers select non-smoking friends, smoking behavior among peer friends becomes similar.

The similarity-attraction hypothesis [94] might offer an explanation why adolescents would select friends based on similar smoking behavior. This theory states that attractiveness of potential friends is associated with similarity in one or more characteristics, for example their smoking behavior or ethnicity. Similarity in such characteristics is presumed to be rewarding as it offers a consensual validation of attitudes and beliefs, and likely will result in enjoyable activities with other adolescents who have similar interests [94]. When smoking behavior similarity holds out the prospect of such rewards, an adolescent might show a higher tendency to choose similar smoking friends.

Social influence of friends occurs when adolescents adjust their smoking behavior in response to the example of or direct pressure by their friends [12-14]. According to the Social Learning Theory, individuals observe, model, and imitate behavior of important individuals in their environment $[15,16]$. As best friends set an example or function as a role model, adolescents are inclined to imitate their best friends' smoking behavior or smoking-related attitudes. Adolescents might also perceive direct pressure to smoke. Direct pressure encompasses behaviors like offering a cigarette, persuasion, challenging or pestering someone to smoke [17].

When studying selection and influence processes among adolescents, it is important to consider social influence of other important individuals in the 
adolescent's social environment such as parents and siblings. Adolescents might model and imitate the smoking behavior of their parents and siblings or feel pressure (not) to smoke. Previous research showed that children are more likely to smoke if one or both of their parents smoke $[18,70]$. The few studies that have examined the effects of sibling smoking also show an association between sibling smoking and adolescent smoking $[18,95]$.

Currently, most smoking prevention programs are based on the assumption that smoking onset is caused by peer smoking and an incapability of adolescents to resist peer pressure [73, 74]. However, mounting evidence suggests that selection processes may be equally or even more important as compared to social influence in explaining smoking behavior similarities among friends $[12,13,23,25,26,28-31,96$, 97]. Consequently, it becomes increasingly important to examine the specific contribution of influence and selection processes to smoking behavior similarity as well as possible changes over time in these processes.

When children grow older they have fewer environmental and parental constraints on their social contacts, gain a longer perspective on their friendships and become more selective about their friends [46]. As social-cognitive skills mature, behavioral and egocentric criteria for selecting and maintaining friends might become less important while sharing of social and emotional experiences gains importance [98]. Children increasingly will use selection criteria that predict friendship durability, relevant for later phases of friendship [44, 46]. Selection of similar smoking friends might therefore decrease during adolescence. To our knowledge, only one study specifically examined influence and selection processes based on smoking behavior over time. Simons-Morton and Chen (2006) found evidence for both influence and selection processes in general, but influence was more consistent over time than selection.

To study influence and selection processes and the changes over time in these processes, the following issues should be taken into account: (1) the method of measuring the smoking behavior of friends, (2) changes in friendship composition, including deselection of friends, and (3) friendship reciprocity. First, adolescents' reports on the number of friends that smoke or on their friends' smoking behavior are often used as friends' smoking behavior measures [12, 26, 28, 29, 31, 32, 97]. This can result in friends' smoking behavior seeming more similar than it actually is, as adolescents may project their own smoking behavior on their friends [35]. By using the smoking behavior as reported by the friends themselves, one can overcome possible projection bias. Second, when longitudinal studies examine social influence 
regarding smoking behavior, deselection processes are often neglected [28, 29]. Only a few studies examined whether deselection of friends was based on smoking behavior $[13,23,24]$. Support for terminating friendships due to dissimilarities in smoking behavior is rarely found, although Ennett and Bauman (1994) did find support for deselection among non-smokers in one study. Although deselection based on dissimilarities in smoking behavior does not seem to play an important role, a biased estimation of social influence may occur when a group of friends still encompasses friends that are not friends anymore at a later time point (former friends). Third, previous research has demonstrated that the social position of an adolescent within a peer group structure is associated with differences in smoking behavior, selection and influence processes [27, 40, 41, 43]. Several studies suggested that social interaction may differ between reciprocal and non-reciprocal friendships $[27,43]$. Reciprocal friendships offer a higher friendship quality, which results in more opportunities for influence processes to cause smoking behavior similarity [27, 43]. Pearson and colleagues considered differences in reciprocity and even demonstrated differences among reciprocal friends that were categorized into relative isolates, group peripherals or group members. The most important targets for selection and influence were adolescents within reciprocal friendships, on the periphery of their peer group [40]. Therefore, it is also important to consider reciprocity when studying selection and influence.

The goal of this article is to examine the specific contribution of influence and selection processes to smoking behavior similarity and changes over time. We will consider possible effects of deselection and examine both non-reciprocal and reciprocal friendships. Based on the theoretical considerations reviewed above, we expect influence and selection processes both to contribute to smoking behavior similarities among adolescent friends. However, the importance of smoking behavior similarity might decrease over time.

\section{METHODS}

\section{Participants}

The sample consisted of 1886 Dutch adolescents that participated as a control group in the European Smoking prevention Framework Approach (ESFA) study [66]. In the ESFA study, communities/regions were randomly chosen, resulting in five 
geographically spread regions within the Netherlands. In each of these regions, schools were randomly selected and assigned to the experimental or control condition according to their own preference. Adolescents that participated in the experimental group were not included, since the smoking prevention intervention was effective in the Netherlands [76], and relationships between variables of interest might also have changed.

\section{Procedure}

Self-administered questionnaires were first distributed among 7th graders in each participating school during autumn 1998. Follow-up was conducted 12, 24 and 30 months later [66]. All students present on the days of data collection were asked to complete the questionnaire. Adolescents were guaranteed that responses would be treated confidentially and that they could refuse to participate. Students returned their questionnaire in sealed envelopes in order to guarantee their anonymity. Cases lost to follow-up were not contacted. In the Netherlands, the proportion of refusals to participate at baseline was $0.56 \%$.

\section{Measures}

Adolescents' weekly smoking behavior was assessed with one question: 'On average, how many cigarettes do you smoke during a week (including the weekend)?' $(0=0 ; 1=$ from 0 to $1 ; 2=2-10 ; 3=11-30 ; 4=>30)$.

Best friends' smoking behavior was assessed primarily by means of a name generator in which each respondent could name up to five 'best friends' inside and/or outside school [81]. Of these friends, only best friends from the same grade at the same school were included, because they were also filling in the questionnaire. Friends outside the school or inside the school, but not in the same grade as the respondents who nominated them were excluded. Cross-gender friendships were allowed, but romantic partners were excluded as it is feasible that romantic partners exert a different influence compared to best friends. The self-reported smoking behavior of each included friend was traced back in their personal questionnaire. In view of the ordinal nature of the respondent's smoking behavior variable, we calculated a median score of the smoking behavior of each respondent's best friends.

Parental smoking behavior was measured by two questions: 'Does your father (male caregiver) smoke?' and 'Does your mother (female caregiver) smoke?'. 
Answers were recoded into one variable $(0=$ neither parent (caregiver) smokes, $1=$ at least one of my parents (caregivers) smokes).

Sibling smoking behavior was measured by two questions: 'Does one or more of your brother(s) smoke?' and 'Does one or more of your sister(s) smoke?', and was recoded into one variable $(0=$ neither siblings smoke, $1=$ at least one of my siblings smokes).

Furthermore, age (years), gender $(0=$ boy, $1=$ girl $)$, ethnicity $(0=$ nonnative, 1 = native), religion ( 0 = not religious, 1 = religious), and alcohol consumption per week, where 1 glass contains approximately 10 grams of pure alcohol $(0=0$ glasses, $1=1$ or 2 glasses, 2 = 3 to 5 glasses, 3 = more than 5 glasses) were measured, which served as covariates in the analysis.

\section{Model development}

To examine social influence and selection we developed an explorative structural equation model which was tested separately in three successive waves. The first wave encompassed baseline data and data of the follow-up 12 months later. The second wave consisted of data from the follow-ups 12 and 24 months later, the third wave was comprised of data from the follow-ups 24 and 30 months later.

Friendship composition changes will be taken into account by distinguishing between future friends, long-standing friends, and former friends at the first measurement of each wave $\left(T_{1}\right)$. Future friends are nominated by the adolescent for the first time at the second measurement of each wave $\left(T_{2}\right)$. Long-standing friends are friends nominated by the adolescent at $T_{1}$ as well as $T_{2}$. Former friends are nominated at $T_{1}$ and not nominated anymore at $T_{2}$. Reciprocity will be taken into account by distinguishing between non-reciprocal and reciprocal friends at $T_{1}$ among future and long-standing friends. Non-reciprocal friends did not confirm the friendship, whereas reciprocal friends did confirm. Figure 1 summarizes the resulting model.

Paths 1 and 2 represent selection. The association between the smoking behavior of non-reciprocal future friends at $T_{1}$ and adolescents at $T_{2}$ (path 1) represents selection of new friends, whereas the association between the smoking behavior of reciprocal future friends at $T_{1}$ and adolescents at $T_{2}$ (path 2) represents selection of new friends that also selected the adolescent as a friend (returning friendship). Paths 3 and 4 represent social influence of non-reciprocal (desired friends) and reciprocal friends, respectively. Of course, selection processes might have been operating before $T_{1}$ to produce these long-standing friendships. The 
association between smoking behavior of former friends at $T_{1}$ and adolescents at $T_{2}$ (path 5), represents deselection based on smoking behavior. Paths 6 and 7 represent the social influence of parents and siblings, respectively. The model furthermore included effects of previous smoking behavior of adolescents, friends, parents and siblings, and covariates of adolescent smoking behavior, so that age, gender, ethnicity, religion, and alcohol consumption were accounted for. The covariates were allowed to influence the smoking behavior of all actors, both at $T_{1}$ and $T_{2}$.

Figure 1 Structural equation model to examine influence and selection processes in each successive data wave

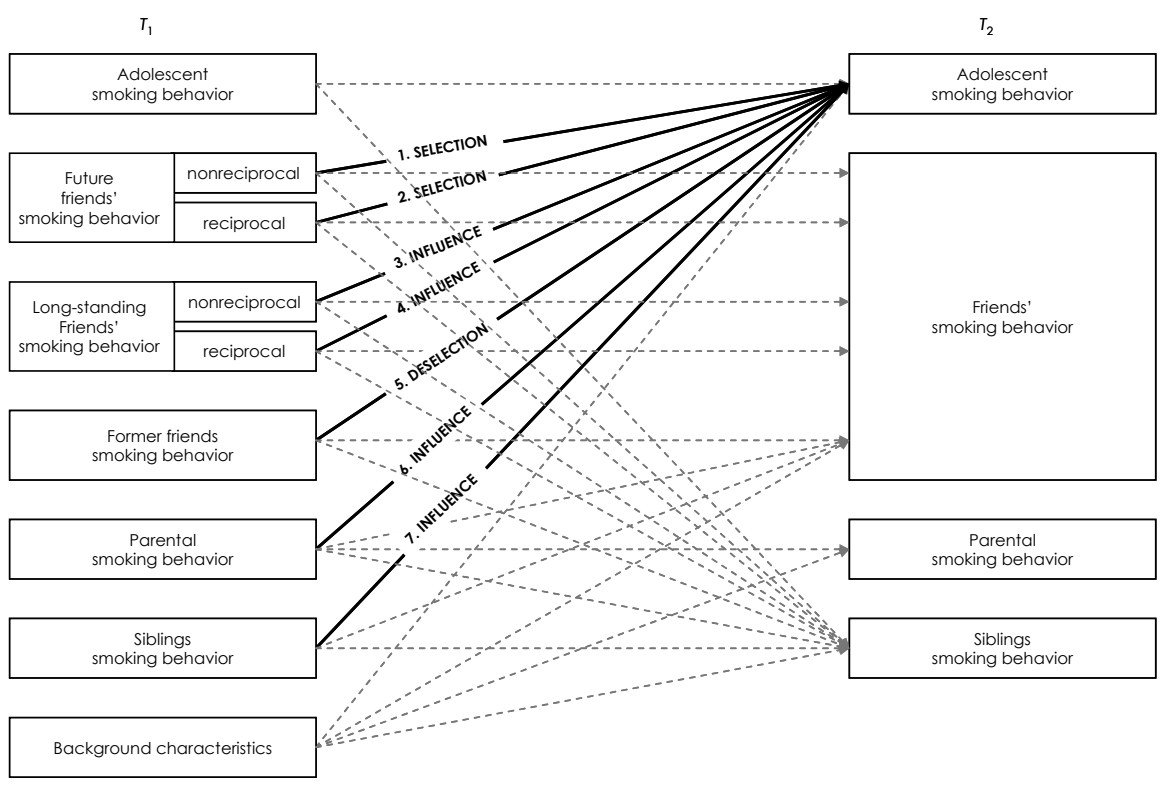

Contemporaneous correlations are included in the model; Background characteristics = age, gender, ethnicity, religion and alcohol consumption; $T_{1}=$ first measurement; $T_{2}=$ second measurement; Bold lines = paths to examine selection and influence processes regarding adolescent smoking behavior; Dotted lines = all other included paths in the model.

\section{Statistical analyses}

To examine selection and influence processes, this model was tested for each of the three waves using structural equation modeling (SEM) employing Mplus 4.1 [82]. Since the variables turned out to be not normally distributed, a robust maximum likelihood (MLR) analysis was performed [83], which produces maximum likelihood parameter estimates and standard errors that are robust to non-normality. The MLR standard errors are obtained through sandwich estimation [84]. MLR is based on all available 
data, not only complete cases, and allows the data to be missing at random (MAR) [82].

Effect sizes were calculated for the seven most important paths (paths 1 to 7) in the model. A measure of effect size is obtained by comparing the -2 log likelihood before and after the inclusion of these paths in the model, and relating this to the -2 log likelihood of a null model. The latter is a model where there are no relationships between the variable to be predicted (adolescent smoking behavior at $T_{2}$ ) and all predictor variables in the model. This measure of effect size can be interpreted as a measure of proportional model fit improvement due to the inclusion of a particular path in the structural equation model [85].

Additional analyses were performed to examine whether results regarding selection and influence within each wave differed significantly from each other. In each wave, selection and influence paths were constrained to be equal. Subsequently, -2 log likelihood values were used to compare model fit between the constrained and unconstrained models. In this chi-square difference test, the -2 log likelihoods were divided by a scaling correction factor to better approximate chisquare under non-normality [99]. Furthermore, the relative contribution of selection and influence will be computed for each wave by taking the ratio of each effect size to the sum of the effect sizes of both selection and influence.

\section{Attrition analyses}

The sample consisted of 1886 Dutch adolescents who completed the questionnaire at baseline. Attrition analyses were performed separately for each of the three waves. In the first wave, 1763 out of 1886 adolescents completed the questionnaire (93.5\%). Logistic regression analysis showed that participation at the second measurement of this first wave was not significantly predicted by any of the included determinants. In the second wave, 1475 out of 1763 respondents (83.7\%) completed the questionnaire at the second measurement. Participation was significantly predicted by deselected friends' smoking behavior and respondents' gender. Respondents with deselected friends that smoked less $(O R=0.995 ; 95 \% \mathrm{Cl}=0.991$ 0.998; $\mathrm{p}<0.01)$, as well as girls $(\mathrm{OR}=1.993 ; 95 \% \mathrm{Cl}=1.408-2.820 ; \mathrm{p}<0.001)$ were more likely to respond. In the third wave, 1366 out of 1475 respondents (92.6\%) completed the questionnaire at the second measurement of this third wave. Respondents with deselected friends that smoked at a lower rate $(O R=0.993 ; 95 \% \mathrm{Cl}$ $=0.988-0.997 ; \mathrm{p}<0.01)$ and girls $(\mathrm{OR}=2.131 ; 95 \% \mathrm{Cl}=1.336-3.397 ; \mathrm{p}<0.01)$ more likely responded. 


\section{RESULTS}

\section{Characteristics of the sample}

Of all 1886 respondents, $91.5 \%$ were non-smokers. Respondents nominated 5705 best friends at baseline out of which 2967 friends (52\%) were in the same school and grade as the respondents who nominated them. Table 1 depicts demographic characteristics of all respondents involved in each wave. The second part of table 1 presents the number of nominated best friends and the percentages of reciprocal and non-reciprocal friends and future friends in each wave at $T_{1}$. The number of total friends increased over time, whereas number of newly selected friends decreased slightly during each follow-up.

Table 1 Background characteristics and nominations of (future) best friends

\begin{tabular}{|c|c|c|c|}
\hline & $\begin{array}{c}\text { Wave } 1 \\
(N=1763)\end{array}$ & $\begin{array}{c}\text { Wave } 2 \\
(N=1475)\end{array}$ & $\begin{array}{c}\text { Wave } 3 \\
(N=1366)\end{array}$ \\
\hline \multicolumn{4}{|l|}{ Background characteristics } \\
\hline Age (mean years) & 12.9 & 13.7 & 14.7 \\
\hline Female gender & $51 \%$ & $52 \%$ & $52 \%$ \\
\hline Nationality: Dutch origin & $77 \%$ & $79 \%$ & $79 \%$ \\
\hline Religious background: Yes & $50 \%$ & $50 \%$ & $50 \%$ \\
\hline \multicolumn{4}{|l|}{ Alcohol consumption per week } \\
\hline No alcohol & $93.4 \%$ & $89.7 \%$ & $78.4 \%$ \\
\hline One or two glasses & $4.9 \%$ & $7.3 \%$ & $13.4 \%$ \\
\hline Three to five glasses & $1.0 \%$ & $1.7 \%$ & $3.4 \%$ \\
\hline$>$ five glasses & $0.7 \%$ & $1.3 \%$ & $4.8 \%$ \\
\hline \multicolumn{4}{|c|}{ Nominated best friends and future best friends } \\
\hline Average number of best friends & 1.6 & 2.2 & 2.3 \\
\hline Non-reciprocal best friends & $59.5 \%$ & $49.4 \%$ & $50.1 \%$ \\
\hline Reciprocal best friends & $40.5 \%$ & $50.6 \%$ & $49.9 \%$ \\
\hline Average number of future best friends & 1.6 & 1.5 & 0.9 \\
\hline Non-reciprocal future best friends & $54.0 \%$ & $57.4 \%$ & $54.9 \%$ \\
\hline Reciprocal future best friends & $46.0 \%$ & $42.6 \%$ & $45.1 \%$ \\
\hline
\end{tabular}

$N=$ number of adolescents, participating at $T_{1}$ in each wave

Table 2 depicts the smoking behavior of adolescents, friends, parents and siblings at $T_{1}$ in each of the three waves. Smoking behavior of adolescents, friends and siblings increased over the three waves, as can be seen by the decreasing percentages of non-smokers among them. Only parental smoking behavior declined slightly. Table 3 shows the correlation matrix for the smoking behavior of respondents, friends, parents, siblings and background characteristics at $T_{1}$ and $T_{2}$ of the first wave. 


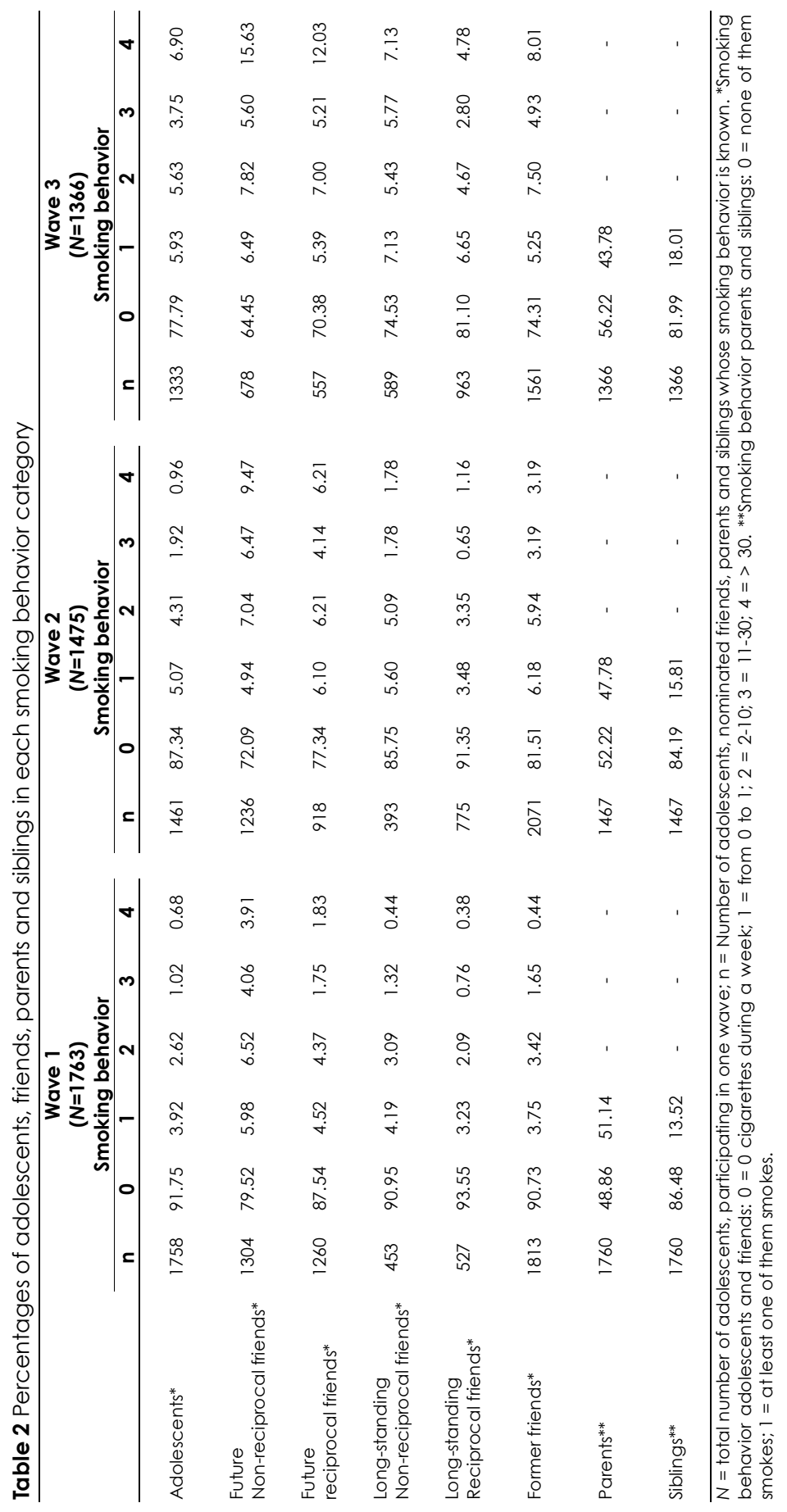




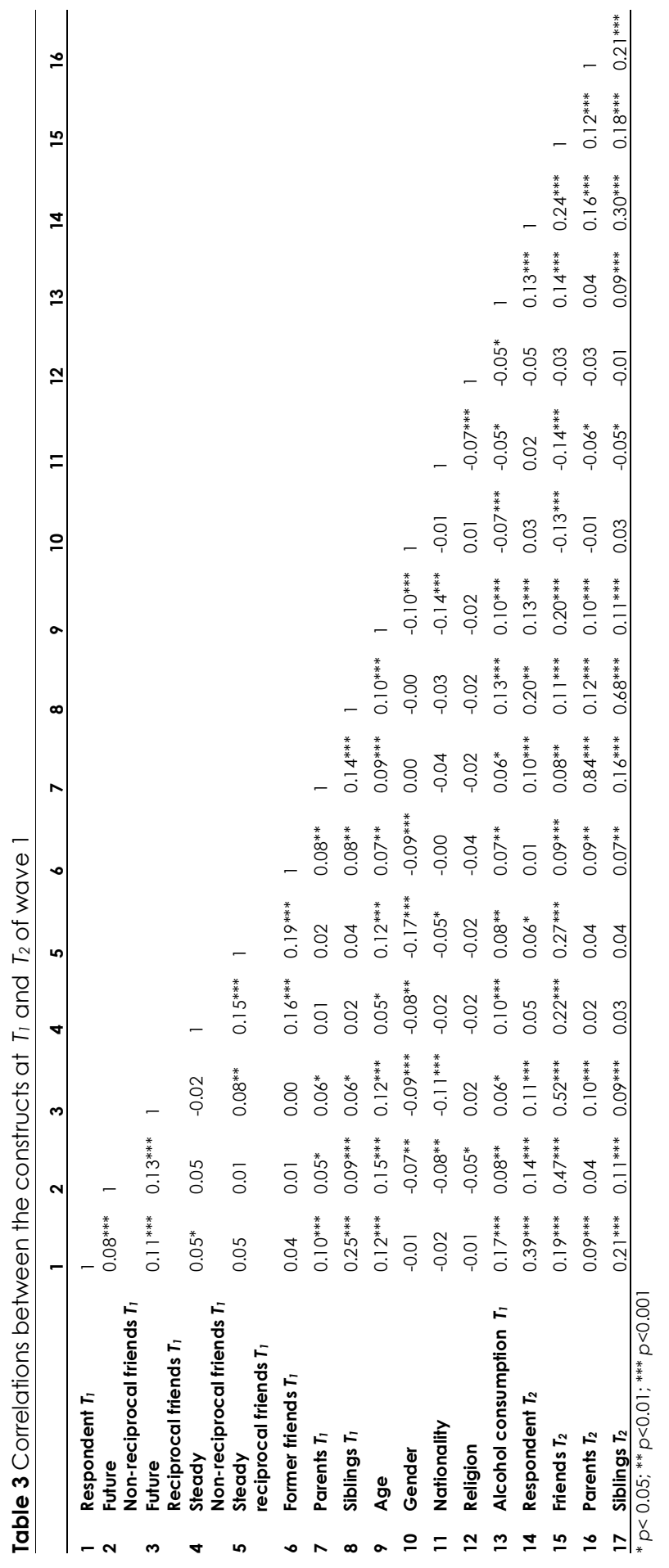




\section{Results from structural equation modeling}

The results of the structural equation modeling (SEM) analyses of the three waves and the proportion of variance explained for each dependent variable in the model are depicted in table 4. Possible school effects were analyzed by including eight dummy variables into the model as covariates. Since no significant school effect was found, the dummy variables were excluded for further analyses.

\section{Selection of friends}

The association between the smoking behavior of non-reciprocal future friends at $T_{1}$ and the respondent at $T_{2}$ (path 1) was positive and significant for wave $1(p<0.001$ ) and wave $2(p<0.01)$, but not for wave $3(p>0.05)$. Hence, only during the first two waves, respondents selected non-reciprocal friends with similar smoking behavior. The association between the smoking behavior of reciprocal future friends at $T_{1}$ and respondents at $T_{2}$ (path 2 ) was positive and significant for wave $1(p<0.01)$, wave 2 $(p<0.001)$, and wave $3(p<0.001)$, indicating that respondents selected reciprocal friends, or returned friendships based on similar smoking behavior during each successive wave.

\section{Influence of friends}

The path coefficient from the smoking behavior of non-reciprocal long-standing friends to the smoking behavior of the respondent at $T_{2}$ (path 3) was positive and significant for wave $3(p<0.001)$. Respondents were not influenced by their nonreciprocal friends in wave 1 and 2 , but adjusted their smoking behavior to become more similar to their non-reciprocal friends in wave 3 . The association between the smoking behavior of reciprocal long-standing friends at $T_{1}$ and respondents at $T_{2}$ (path 4) was not significant for any of the three waves $(p>0.05)$, indicating that respondents were not influenced by their reciprocal friends. The association between the smoking behavior of former friends at $T_{1}$ and respondents at $T_{2}$ (path 5) was not significant in the three waves $(p>0.05)$. Hence, respondents were not influenced by their former friends.

\section{Influence of parents and siblings}

The effect of parental smoking behavior at $T_{1}$ on respondents' smoking behavior at $T_{2}$ (path 6) was only significant in wave $2(p<0.01)$. Adolescents with smoking parents, smoked more cigarettes. The effect of sibling smoking behavior on respondents' smoking behavior at $T_{2}$ (path 7) was positively significant for wave $1(p<0.01)$, and 
wave 2 ( $p<0.01)$. Adolescents having at least one smoking sibling, smoked more compared to adolescents having no smoking siblings.

\section{Previous smoking behavior and covariates}

Smoking behavior of adolescents, friends, parents and siblings, were significantly predicted by their own previous behavior in all three waves $(p<0.05)$. Of the covariates assessed, only alcohol consumption was associated with respondents' smoking behavior at $T_{2}$ in wave 1 (coefficient $=0.571$; $p<0.01$ ), wave 2 (coefficient $=$ $0.331 ; p<0.05$ ), and wave 3 (coefficient $=0.253 ; p<0.05$ ). Respondents who drank more alcohol smoked significantly more cigarettes.

\section{Relative contributions of selection and influence}

To examine whether results regarding selection and influence of friends within each wave differed significantly from each other, additional analyses were performed. Within non-reciprocal friendships, significant differences were found between the regression weights of selection and influence in wave 1 (Chi-square ( $d f=1$ ) $=7.95$ ) as well as wave 3 (Chi-square $(\mathrm{df}=1)=5.29)$. Selection based on similar smoking behavior contributed significantly more to smoking behavior similarity between friends compared to social influence from friends during the first wave. During the third wave, social influence from friends contributed significantly more to smoking behavior similarity compared to selection. The relative contributions of selection and influence among non-reciprocal friends were respectively $97.71 \%$ and $2.29 \%$ in wave one, $77.78 \%$ and $22.22 \%$ in wave 2 , and $10.71 \%$ and $89.29 \%$ in wave 3 . Within reciprocal friendships, no significant differences were found between the regression weights of selection and influence. Hence selection processes seem to be more profound in early adolescence, whereas influence processes become more important during mid adolescence. 
Table 4 Results of Structural Equation Modeling using robust maximum likelihood for each wave separately: path coefficients (with standard errors), significance levels and effect sizes

\begin{tabular}{|c|c|c|c|c|c|c|}
\hline \multirow[b]{2}{*}{ ADOLESCENT'S SMOKING BEHAVIOR $T_{2}$ on } & \multicolumn{2}{|c|}{$\begin{array}{c}\text { Wave 1 } \\
(N=1763)\end{array}$} & \multirow{2}{*}{\multicolumn{2}{|c|}{$\begin{array}{c}\begin{array}{c}\text { Wave 2 } \\
(N=1475)\end{array} \\
\text { Estimate (s.e.) }\end{array}$}} & \multicolumn{2}{|c|}{$\begin{array}{c}\text { Wave } 3 \\
(N=1366)\end{array}$} \\
\hline & Estimate & (s.e.) & & & Estimate & (s.e.) \\
\hline Non-reciprocal future friends' smoking & $0.320^{* * *}$ & $(0.050)$ & $0.113^{* *}$ & (0.039) & 0.048 & $(0.042)$ \\
\hline (1. selection) & \multicolumn{2}{|c|}{$E S=0.128$} & \multicolumn{2}{|c|}{$E S=0.021$} & \multicolumn{2}{|c|}{$E S=0.003$} \\
\hline Reciprocal future friends' smoking & $0.238^{* *}$ & $(0.070)$ & $0.357^{* * *}$ & (0.048) & $0.167^{* * *}$ & (0.045) \\
\hline (2. selection) & \multicolumn{2}{|c|}{$E S=0.038$} & \multicolumn{2}{|c|}{$E S=0.180$} & \multicolumn{2}{|c|}{$E S=0.031$} \\
\hline Non-reciprocal long-standing friends' smoking & -0.226 & $(0.188)$ & 0.191 & $(0.126)$ & $0.226^{* * *}$ & (0.066) \\
\hline (3. influence) & \multicolumn{2}{|c|}{$E S=0.003$} & \multicolumn{2}{|c|}{$E S=0.006$} & \multicolumn{2}{|c|}{$E S=0.025$} \\
\hline Reciprocal long-standing friends' smoking & 0.341 & $(0.228)$ & 0.264 & $(0.140)$ & 0.075 & (0.064) \\
\hline (4. influence) & \multicolumn{2}{|c|}{$E S=0.005$} & \multicolumn{2}{|c|}{$E S=0.012$} & \multicolumn{2}{|c|}{$E S=0.003$} \\
\hline Former friends' smoking & -0.072 & $(0.218)$ & 0.071 & $(0.070)$ & -0.097 & $(0.062)$ \\
\hline (5. deselection) & \multicolumn{2}{|c|}{$E S=0.000$} & \multicolumn{2}{|c|}{$E S=0.002$} & \multicolumn{2}{|c|}{$E S=0.006$} \\
\hline Parental smoking & 0.197 & $(0.152)$ & $0.409 * *$ & $(0.153)$ & 0.134 & $(0.149)$ \\
\hline (6. influence) & \multicolumn{2}{|c|}{$E S=0.004$} & \multicolumn{2}{|c|}{$E S=0.019$} & \multicolumn{2}{|c|}{$E S=0.002$} \\
\hline Siblings' smoking & $0.687^{* *}$ & (0.199) & $0.643^{* *}$ & $(0.206)$ & 0.250 & $(0.178)$ \\
\hline (7. influence) & & $=0.034$ & & $=0.030$ & & $=0.004$ \\
\hline Adolescent's smoking & $0.940^{* * *}$ & $(0.135)$ & $0.858^{* * *}$ & $(0.112)$ & $1.284^{* * *}$ & $(0.095)$ \\
\hline Adolescent's alcohol consumption $^{\circ}$ & $0.571^{* *}$ & $(0.213)$ & $0.331^{*}$ & $(0.158)$ & $0.253^{*}$ & (0.103) \\
\hline FRIENDS' SMOKING BEHAVIOR $T_{2}$ on & & & & & & \\
\hline Non-reciprocal future friends' smoking & $0.810^{* * *}$ & (0.073) & $0.810^{* * *}$ & $(0.070)$ & $0.748^{* * *}$ & (0.074) \\
\hline Reciprocal future friends' smoking & $0.939 * * *$ & $(0.088)$ & $0.974^{* * *}$ & $(0.075)$ & $0.757^{* * *}$ & (0.086) \\
\hline Non-reciprocal long-standing friends' smoking & $0.542^{*}$ & $(0.268)$ & $0.660^{* * *}$ & $(0.191)$ & $0.737^{* * *}$ & $(0.128)$ \\
\hline Reciprocal long-standing friends' smoking & $0.853^{* * *}$ & $(0.218)$ & $0.908^{* * *}$ & $(0.158)$ & $0.635^{* * *}$ & (0.094) \\
\hline Former friends' smoking & 0.099 & $(0.396)$ & -0.056 & $(0.123)$ & 0.073 & $(0.105)$ \\
\hline Adolescent's smoking & 0.073 & $(0.170)$ & -0.342 & $(0.178)$ & 0.005 & $(0.115)$ \\
\hline Parental smoking & 0.252 & $(0.202)$ & 0.053 & (0.224) & $-0.780^{* *}$ & $(0.251)$ \\
\hline Siblings' smoking & 0.092 & $(0.281)$ & -0.293 & $(0.312)$ & -0.271 & (0.326) \\
\hline Adolescent's race & 0.108 & $(0.237)$ & $-0.647^{*}$ & $(0.277)$ & 0.352 & (0.326) \\
\hline Adolescent's religion & 0.323 & $(0.207)$ & $0.458^{*}$ & $(0.214)$ & 0.271 & (0.232) \\
\hline Adolescent's alcohol consumption & 0.271 & $(0.221)$ & $0.424^{*}$ & $(0.176)$ & 0.036 & $(0.134)$ \\
\hline PARENTAL SMOKING BEHAVIOR $T_{2}$ on & & & & & & \\
\hline Parental smoking & $5.245^{* * *}$ & (0.195) & $4.870^{* * *}$ & $(0.205)$ & $4.663^{* * *}$ & $(0.200)$ \\
\hline Adolescent's gender & -0.118 & $(0.190)$ & $0.403^{*}$ & $(0.187)$ & -0.095 & (0.190) \\
\hline Adolescent's religion & -0.266 & (0.192) & $0.479 *$ & (0.189) & $-0.605^{* *}$ & $(0.191)$ \\
\hline SIBLINGS' SMOKING BEHAVIOR $T_{2}$ on & & & & & & \\
\hline Non-reciprocal future friends' smoking & 0.113 & (0.066) & -0.012 & (0.055) & $0.124^{*}$ & $(0.062)$ \\
\hline Reciprocal future friends' smoking & $0.278 * * *$ & (0.074) & $0.185^{* *}$ & (0.067) & 0.118 & (0.073) \\
\hline Non-reciprocal long-standing friends' smoking & 0.004 & $(0.274)$ & -0.045 & $(0.129)$ & -0.014 & (0.098) \\
\hline Reciprocal long-standing friends' smoking & 0.419 & (0.227) & 0.073 & $(0.106)$ & $-0.255^{*}$ & $(0.126)$ \\
\hline Former friends' smoking & $-0.639 *$ & $(0.280)$ & -0.003 & $(0.120)$ & 0.094 & $(0.081)$ \\
\hline Adolescent's smoking & 0.112 & (0.168) & 0.133 & (0.139) & 0.126 & $(0.121)$ \\
\hline Parental smoking & $0.697^{* * *}$ & $(0.193)$ & 0.026 & $(0.207)$ & $0.448^{*}$ & $(0.212)$ \\
\hline Siblings' smoking & $4.598^{* * *}$ & $(0.256)$ & $4.170^{* * *}$ & $(0.229)$ & $4.560^{* * *}$ & $(0.247)$ \\
\hline Loglikelihood & -18956.995 & & -18838.02 & & -18865.140 & \\
\hline R-square adolescent's smoking behavior & 0.164 & & 0.245 & & 0.361 & \\
\hline R-square friends' smoking behavior & 0.472 & & 0.648 & & 0.714 & \\
\hline R-square parental smoking behavior & 0.515 & & 0.477 & & 0.454 & \\
\hline R-square siblings' smoking behavior & 0.338 & & 0.297 & & 0.370 & \\
\hline
\end{tabular}




\section{DISCUSSION}

The goal of this article was to examine selection and influence effects on adolescent smoking behavior and changes in these processes during early and mid adolescence. Although the smoking rates of adolescents in the present study were high, they were comparable to smoking rates of youngsters in many Western countries [4]. Four main conclusions can be drawn.

First, findings showed that selection as well as influence processes are important in explaining smoking behavior similarities among adolescent friends. In line with a previous study [96], respondents selected non-reciprocal and reciprocal friends based on similar smoking behavior. Furthermore, adolescents were only influenced by their non-reciprocal friends to adopt similar smoking behavior. We did not find evidence for influence of reciprocal friends. These findings are in line with a previous study, in which desired (non-reciprocal) friends exerted an important influence on smoking behavior. Desired friends were more likely to reward an adolescent with reciprocity if the adolescent's smoking behavior was consistent with his desired friends' than if it was inconsistent [100]. This is contrary to Urberg and colleagues (2003) who argued that higher friendship quality and more interaction opportunities resulted in more opportunities for social influence processes to cause similarity of smoking behavior. However, since adolescents in our study were slightly younger, it is still possible that influence processes might have increased also among reciprocal friends after 9th grade. More research is needed to investigate selection and influence processes over longer time periods.

Second, the importance of selection and influence processes in explaining smoking behavior similarity changed from early to mid adolescence. When adolescents grew older, smoking-based selection processes decreased while the importance of influence processes increased. Smoking behavior similarity is an important criterion when selecting new friends during early adolescence. With age, however, its importance seems to decline. For selection of non-reciprocal friends, the importance of smoking behavior similarity diminished. Criteria for friend selection are known to change over time. As adolescents grow older, they may start to use criteria that are more relevant for later phases of friendship, which predict later satisfaction and durability of friendship [46]. The importance of influence processes among friends increased from early to mid adolescence. Adolescents were increasingly influenced by the smoking behavior of their non-reciprocal friends. During mid adolescence, children started changing their own smoking behavior to become 
more similar to their non-reciprocal friends, at the moment that non-reciprocal friends were not chosen anymore based on similar smoking behavior. Since smoking behavior similarity is not a selection criterion anymore for choosing a non-reciprocal friend, but keeps on being an important criterion for choosing a reciprocal friend or returning friendships, adolescents start to change their smoking behavior to become more similar to their desired friends. Changing the smoking behavior to become more similar will maximize the chance of reciprocity as long as smoking behavior similarity keeps on being an important criterion for returning friendships during mid adolescence. Hence, it is possible that influence processes causing smoking behavior similarity are triggered by the decrease in selection based on similar smoking behavior. If older adolescents still want to maximize their chances for reciprocity, they have to change their strategy and start to adopt their behavior to become more similar to their desired friends.

Third, the lack of evidence for influence processes among reciprocal friends might suggest that, regardless of age, smoking behavior similarity is more important during the earlier phases of friendship formation. Selection of non-reciprocal or reciprocal friends, and having non-reciprocal friends, might be regarded as initial phases of friendship formation, while sharing an established long-standing friendship might be regarded as a later or maybe even last phase of the friendship formation process. Previous research already demonstrated that similarity appeared to be relatively more important during the initial phases of friendship formation, when individuals choose potential friends. In later phases, when they establish long-lasting friendships, the provision of social and emotional resources such as companionship, emotional support, help and self-validation, become more important [46], and thus allow for more dissimilarity in certain behaviors.

Fourth, although no support was found for influence processes of friends during early adolescence, social influence of parents and siblings did play an important role in early adolescent smoking behavior. During the first wave adolescents' smoking behavior was mainly influenced by the smoking behavior of their siblings, while during the second wave both siblings and parents exerted an important influence. The effects of parental and sibling smoking status declined during the last wave. These findings are in line with previous research among adolescents reporting a decrease of interaction with parents [101], lower feelings of acceptance by parents, and less satisfaction with family life [102]. 


\section{Limitations}

Besides several strengths of this study such as the longitudinal design, the inclusion of parents, siblings as well as best friends, and the consideration of several important methodological issues, there are also limitations. First, in the ESFA study, five Dutch regions were randomly selected, in which schools were assigned to the experimental or control condition according to own preference, which may have resulted in some bias in the distribution of adolescents' characteristics. Although matching on degree of urbanization and educational level was used to make schools comparable, future studies should try to replicate our findings in a sample collected through random assignment of schools to the treatment groups. Second, the use of a fixed-response name generator might have restricted the ability to reciprocate, as respondents were only allowed to nominate five best friends. However, previous research, allowing 7th graders to nominate any number of friends, showed that on average 4.09 friends were nominated [88]. Moreover, allowing nominating more than five best friends might provoke adolescents to nominate peers who are not 'best' friends. Third, only friends inside school in the same grade were included since only those nominated friends also filled in the same questionnaire including their self-reported smoking behavior. Therefore, our study could not examine potential influences of friends outside school or inside school but in a different grade. Future studies should aim to include influences of other friends as well. Fourth, respondents' self-reported smoking behavior was not validated by biochemical measures. However, self-reports have been shown to be reliable and to correspond well with biological indicators when measurements are done under optimized measurement conditions that assure anonymity [86]. During the ESFA project, strict confidentiality of adolescent responses was guaranteed [66]. Fifth, no direct measures of parental and sibling smoking behavior were available. The effects of parental and sibling smoking behaviors might have been overestimated, although previous research has demonstrated that adolescents aged 13-17 years are reliable sources for assessing the smoking status of their parents [103]. Finally, SEM techniques assume that dependencies caused by the network structure of an adolescent do not exist. In our study, a given individual's data could appear within more than one observation, e.g. as the smoking behavior outcome for a given case and as one of the friends supplying data for other individual cases. Furthermore, adolescents can be subject not only to influences of multiple friends but also to potentially important indirect 'friend-of-friend' influences. New social network analysis methods are currently being developed which are able to take these kinds of dependencies into account [62]. 


\section{Practical Implications}

This study has several practical implications. First, friendship composition changes, including deselection of friends, should be taken into account in future studies examining influence processes. Not considering or controlling for deselection might lead to an underestimation of influence processes among friends, as our results showed that smoking behavior of former friends did not influence adolescent's present smoking behavior. Second, our findings suggest that future research should consider the social position of an adolescent within a peer group structure since selection and influence processes differed within non-reciprocal and reciprocal friendships. Suggestions for the development of smoking prevention among adolescents can also be inferred from the current study. Smoking prevention programs should focus on selection processes besides social influence processes. In line with previous research we emphasized that the way peer environments are structured needs more attention within prevention programs besides the promotion of social influence skills [90-92]. Programs should also draw on different strategies for early and mid adolescence. During early adolescence, when youngsters start forming new friendships, adolescents were mainly influenced by smoking behavior of their parents and siblings. During mid adolescence, this influence diminished and peer smoking behavior increasingly affected adolescent smoking behavior. Programs targeting youngsters in early adolescence should make parents as well as siblings aware of the effects of their own smoking behavior upon the young adolescent. During mid adolescence, our results suggest a different approach targeting mainly the youngsters themselves. Adolescents should be made aware of the effects of peer smoking behavior. Adolescents have to learn not to adjust to desired friends. In addition, by making adolescents aware of how friendships are formed and which characteristics predict a stable long-lasting friendship, programs might also prevent adolescents from returning friendships based on similar smoking behavior. 



\section{CHAPTER 4}

Social network analyses in six European countries

Based on: Mercken, L., Snijders, T., Steglich, C., \& De Vries, H. (2009) Dynamics of friendship networks and smoking behavior: social network analyses in six European countries. Social Science \& Medicine (In press). 


\section{ABSTRACT}

The co-evolution of adolescents' friendship networks and their smoking behavior is examined in a large sample across six European countries. Selection and influence processes are disentangled using new methods of social network analysis that enable alternative selection mechanisms to be controlled for. The sample consisted of 7704 adolescents participating in the control group of the ESFA (European Smoking prevention Framework Approach) study. The design was longitudinal with four measurements. Results indicated that in each country adolescents preferred selecting friends based on similar smoking behavior. Support for influence of friends was found in only two countries. Similarity of smoking behavior between friends was explained more strongly by smoking-based selection processes than by influence of friends in each of the six countries. Prevention programs need to address aspects that drive peer selection, and reinforce non-smoking attitudes in adolescents. 


\section{INTRODUCTION}

Although smoking prevention programs yield short-term effects, the long-term effectiveness is often modest [2, 73, 104]. One explanation may be that the assumption that smoking onset is caused by peer influences $[73,74]$ is only partly valid. Although smoking behavior tends to be similar among friends $[8,10,11]$, this similarity can also be attributed to the selection of similar others, instead of influence $[12,13,23,29,96,105]$.

Although previous studies of peer influence on smoking onset successfully controlled for other determinants of smoking, such as age, gender, parental and sibling smoking [18], they failed to control for other determinants of friendship selection besides smoking-based selection of friends. Some of these other determinants are reciprocation of friendship; to become a friend of somebody who already is a friend of a friend [106, 107]; and similarities in other variables such as alcohol consumption [89], age, ethnicity, education, or gender [52]. All of these other determinants may also lead to similarity of friends with respect to smoking behavior, and failing to control for such alternative selection processes may accordingly result in an overestimation of selection based on similar smoking behavior.

Furthermore, previous studies did not account for unobserved changes in friendships and smoking behavior between the measurement moments. Longitudinal data is mostly gathered at discrete moments, which makes it impossible to unequivocally identify which process is responsible for a network or behavioral change. In between two observations, changes will occur in friendship and smoking behavior, and a change may even be followed by a change back to the original value before the next observation. Figure 1 demonstrates a process diagnosed as influence on the basis of discrete observations, but for which the sequence of events could have been different, up to the point that it could have been a case of selection based on similar smoking behavior when observed continuously.

This study examined smoking-related friendship selection and friends' influence within the same school grade, while controlling for alternative explaining selection mechanisms. Although data were gathered at four discrete moments in time, continuous changes in friendships and smoking behavior in between two observations were modeled using newly developed methods of social network analysis [62, 89]. These methods employ a more complete representation of repeated measures data on friendship networks and smoking behavior, and allow 
the parameters that influence selection processes to be estimated and controlled for. The sample included adolescents across six countries in Northern, Central, and Southern Europe, permitting better generalization than data from just one country. Based on previous studies we hypothesized that similarities in smoking behavior among friends within the same school grade would be attributed to both selection and influence processes. We furthermore expected stronger selection processes than peer influence processes to explain the observed similarity of smoking behavior between friends.

Figure 1 Ambiguity in diagnosing the peer influence process

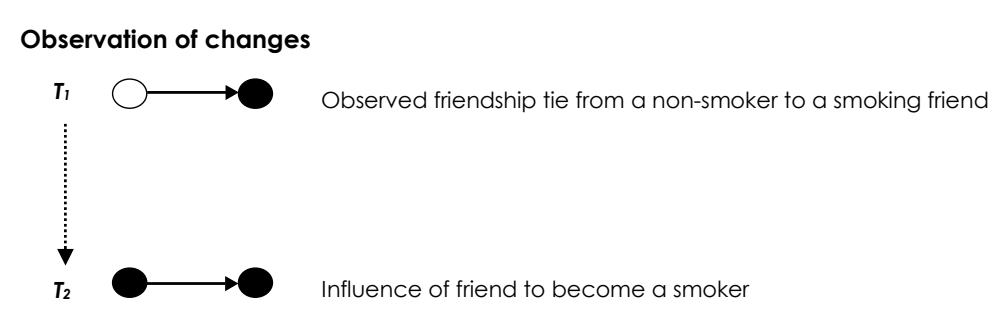

\section{Possible sequence of changes between observations}

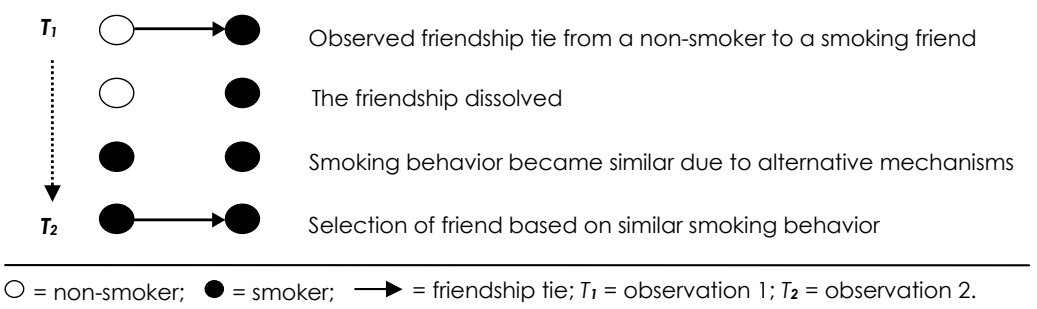




\section{METHODS}

\section{Participants}

The sample consisted of 7704 adolescents from six European countries (Denmark, Finland, the Netherlands, Portugal, the United Kingdom (UK), and Spain) that participated in the European Smoking prevention Framework Approach (ESFA) study [66]. Communities (or regions) were randomly selected in each country. High schools were asked to participate, with a $50 \%$ chance of becoming an experimental school. Experimental schools were excluded from the current study because the intervention may have changed the relationship between variables of interest. This study included all control schools that participated at the four measurement times: 17 Danish schools $(N=843), 11$ Finnish schools $(N=1326), 9$ Dutch schools $(N=2524), 8$ Portuguese schools $(N=1590), 4$ UK schools $(N=792)$, and 21 Spanish schools $(N=629)$. In none of the countries did school transitions occur during the ESFA project. Table 1 demonstrates the average school network structure and demographic characteristics. The average number of friends decreased slightly over time, while smoking behavior increased.

\section{Procedure}

Self-administered questionnaires were distributed in the seventh grade (mean age = 13) of each participating school during autumn 1998. Follow-up was conducted at 12, 24, and 30 months [76]. Parents were informed about the ESFA study and could refuse to have their child participate. All students present on the days of data collection were asked to complete the questionnaire. Adolescents were informed that responses would be treated confidentially and that they too could refuse to participate. Students returned their questionnaires in sealed envelopes to guarantee confidentiality. The overall percentage of refusals to participate was $1.710 .6 \%$ in Denmark, $3.0 \%$ in Finland, $0.6 \%$ in the Netherlands, $2.2 \%$ in Portugal, $1.0 \%$ in Spain, and $2.3 \%$ in the UK).

\section{Questionnaire}

Friendship ties were assessed by one question in which adolescents could name up to five best friends inside and/or outside school [81]. Only best friends inside school in the same grade were included here, since only best friends also participated as respondents and filled out the questionnaire. 
Smoking behavior of adolescents was assessed by one question: "On average, how many cigarettes do you smoke during a week (also count the weekend)?" $(0=0,1=$ between 0 and 1, $2=2-10,3=11-30,4=>30)$.

Parental smoking behavior was measured by two questions: 'Does your father (male caregiver) smoke?' and 'Does your mother (female caregiver) smoke?', and was recoded into one variable $10=$ neither smokes, $1=$ at least one parent (caregiver) smokes).

Sibling smoking behavior was measured by two questions: 'Does one or more of your brother(s) smoke?' and 'Does one or more of your sister(s) smoke?', and was recoded into one variable ( $0=$ no siblings smoke, $1=$ at least one sibling smokes). For those adolescents who indicated having no siblings, the answer was treated as missing.

Alcohol consumption ( $0=0$ glasses of alcoholic drinks per week, $1=1$ or 2 glasses, 2 = 3 to 5 glasses, $3=$ more than 5 glasses), age (in years), gender $(0=$ boy, 1 = girl), nationality ( 0 = non-native, $1=$ native), and self-reported school achievement ( 1 = among the lower third of the class, 2 = the middle third, $3=$ the highest third) were also recorded.

\section{Model development}

The interdependence between changes in friendship ties, and between changes in friendships and smoking behavior, requires a model that expresses this dependence in a plausible way. To examine selection and influence a model was constructed which consists of two parts: one part modeled friendship network changes (selection processes), another part modeled smoking behavior changes (influence processes). The combined model examines selection and influence processes simultaneously while controlling either process for the other one. To model continuous changes between discrete observations, continuous-time Markov chains were used. This implies that changes in friendship choices or smoking behavior were assumed to depend on the current state of friendship and smoking, not on the more distant past (Markov property). The mathematical specification and statistical estimation procedures are given by Snijders et al. $[62,64]$ and the model specification was as follows.

\section{Friendship network changes: selection processes}

The friendship network evolution part of the model includes functions of current network structure and adolescents' attributes that determine friendship choice 
probabilities. These were called 'effects' and are presented in the upper part of Table 2. This list contained three smoking-based friendship selection components: the effect of smoking behavior on number of friends chosen (smoking behavior adolescent), the effect of potential friends' smoking behavior (smoking behavior potential friend), and the interaction effect between these two, which was used to test whether adolescents who smoke more also prefer friends who smoke more (smoking behavior adolescent $\times$ potential friend). As friendship choices might depend on characteristics of the current network $[52,64,108]$, the effects of number of friends chosen (outgoing friendships), number of reciprocal friends chosen (reciprocity), and number of friends chosen who were also a friend of a friend (transitivity) were included. Furthermore, selection based on alcohol consumption, age, gender, nationality, and school achievement of adolescents and potential friends was controlled for (see Table 2 for a complete overview).

\section{Smoking behavior changes: influence processes}

The smoking behavior evolution part of the model included a list of functions of network, smoking behavior, and other attributes on which probabilities of changes in smoking behavior may depend. These effects are presented in the lower part of Table 2. The list contained three friendship network-related influence components: the effect of smoking behavior of friends on adolescent smoking; the effect of number of received friendship nominations (incoming friendships/popularity), and the effect of number of outgoing friendship nominations on smoking (outgoing friendships). Included control effects were the tendency to smoke, the addictive 'feedback' effect of smoking, parental and sibling smoking, and adolescents' alcohol consumption, age, gender, nationality, and school achievement. Adequately controlling for attributes resulted in a larger number of effects included in the friendship evolution part of the model compared to the smoking behavior evolution part. This difference was due to the multidimensional nature of selection processes. For example, while the effect of gender on adolescent smoking can be modeled by one influence parameter (the effect of adolescents' gender on own smoking behavior), the effect of gender on friendship can be modeled by three different selection features: the gender of the adolescent, the gender of the potential friend, and the similarity in gender of the adolescent and the potential friend. 
Table 1 Descriptive statistics of network structure of schools and individual characteristics

\begin{tabular}{|c|c|c|c|c|c|c|}
\hline Country & $\begin{array}{r}\text { DK } \\
(N=843) \\
\end{array}$ & $\begin{array}{r}\mathrm{FN} \\
(N=1326) \\
\end{array}$ & $\begin{array}{r}N L \\
(N=2524) \\
\end{array}$ & $\begin{array}{r}P O \\
(N=1590) \\
\end{array}$ & $\begin{array}{r}\text { UK } \\
(N=792) \\
\end{array}$ & $\begin{array}{r}S P \\
(N=629) \\
\end{array}$ \\
\hline \multicolumn{7}{|c|}{ Average network structure of schools } \\
\hline Average number of adolescents & 50 & 121 & 280 & 199 & 198 & 30 \\
\hline \multicolumn{7}{|l|}{ Average number of joiners } \\
\hline Period 1 & 7 & 8 & 24 & 30 & 27 & 4 \\
\hline Period 2 & 4 & 9 & 36 & 25 & 7 & 2 \\
\hline Period 3 & 3 & 2 & 10 & 5 & 9 & 0 \\
\hline \multicolumn{7}{|l|}{ Average number of leavers } \\
\hline Period 1 & 4 & 8 & 14 & 38 & 15 & 2 \\
\hline Period 2 & 8 & 10 & 43 & 39 & 25 & 4 \\
\hline Period 3 & 7 & 20 & 22 & 14 & 33 & 2 \\
\hline \multicolumn{7}{|c|}{ Average number of friendship nominations } \\
\hline Wave 1 & 2.4 & 1.7 & 1.5 & 1.4 & 2.1 & 2.2 \\
\hline Wave 2 & 2.3 & 2.0 & 1.7 & 1.5 & 2.7 & 2.2 \\
\hline Wave 3 & 2.2 & 1.8 & 1.7 & 1.5 & 2.6 & 2.0 \\
\hline Wave 4 & 2.2 & 1.8 & 1.6 & 1.5 & 2.3 & 1.8 \\
\hline \multicolumn{7}{|c|}{ Average observed network similarity a } \\
\hline Wave 1 & 0.2 & 0.4 & 0.2 & 0.1 & 0.1 & 0.0 \\
\hline Wave 2 & 0.4 & 0.4 & 0.3 & 0.4 & 0.3 & 0.3 \\
\hline Wave 3 & 0.5 & 0.4 & 0.3 & 0.2 & 0.5 & 0.2 \\
\hline Wave 4 & 0.3 & 0.4 & 0.3 & 0.3 & 0.4 & 0.3 \\
\hline
\end{tabular}

\section{Individual characteristics}

Smoking behavior adolescent (0-4)

\begin{tabular}{|c|c|c|c|c|c|c|}
\hline Wave 1 & 0.4 & 0.5 & 0.2 & 0.2 & 0.2 & 0.1 \\
\hline Wave 2 & 0.8 & 0.9 & 0.5 & 0.4 & 0.6 & 0.5 \\
\hline Wave 3 & 1.1 & 1.4 & 0.8 & 0.6 & 0.9 & 0.8 \\
\hline Wave 4 & 1.2 & 1.4 & 0.9 & 0.7 & 1.0 & 1.2 \\
\hline consumption adolescent (0-3) & 0.3 & 0.3 & 0.3 & 0.2 & 0.5 & 0.1 \\
\hline baseline (in years) & 13.3 & 13.4 & 12.8 & 13.2 & 12.6 & 12.4 \\
\hline tage females & 50.0 & 47.0 & 49.0 & 47.0 & 64.0 & 47.0 \\
\hline tage Natives & 97.0 & -- & 75.0 & 87.0 & 98.0 & 95.0 \\
\hline achievement (1-3) & 2.3 & 2.0 & -- & 2.2 & 2.2 & 2.1 \\
\hline tage at least one smoking parent & 63.7 & 49.9 & 50.2 & 51.4 & 38.0 & 63.2 \\
\hline tage at least one smoking sibling & 25.8 & 23.0 & 19.3 & 19.0 & 18.5 & 23.3 \\
\hline
\end{tabular}

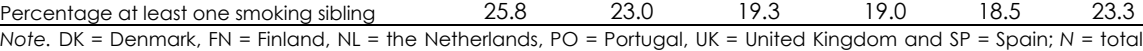
number of adolescents participating in at least one wave in the school networks. Smoking behavior is coded: $0=0$ cigarettes each week; $1=$ between 0 and $1 ; 2=2-10 ; 3=11-30 ; 4=>30$; Alcohol consumption is coded: $0=0$ glasses alcohol each week; $1=1-2 ; 2=3-5 ; 3=>5$; School achievement is coded: $1=$ among the lower third of the class; $2=$ middle third; $3=$ best third. --= variable was not measured. a Network autocorrelation coefficient: Moran's I. 
CHAPTER 4 | SOCIAL NETWORK ANALYSES IN SIX EUROPEAN COUNTRIES

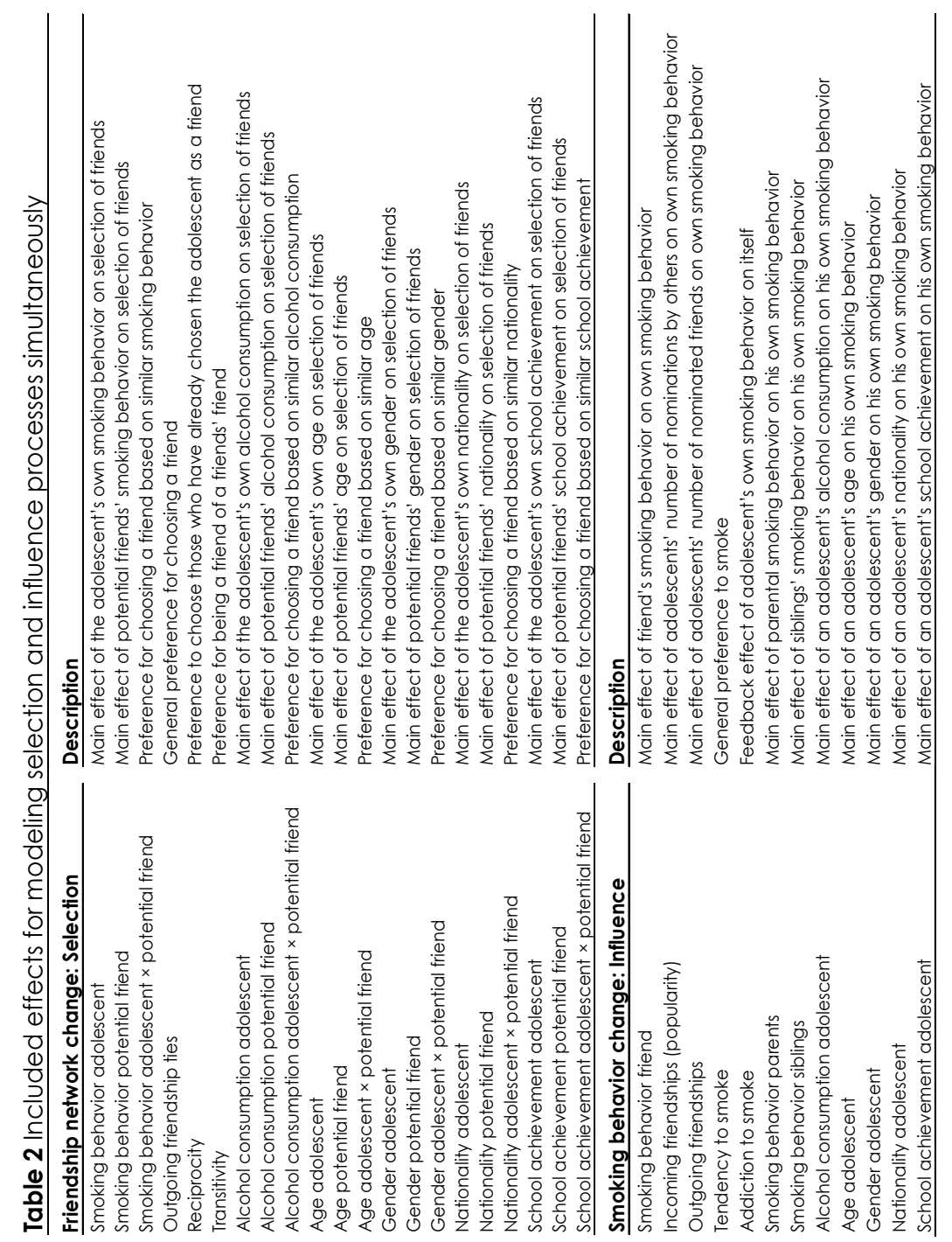




\section{Analysis}

The proposed combined model was analyzed for each school separately using SIENA (Simulation Investigation for Empirical Network Analysis) software [109]. Effects were tested on the basis of $t$-ratios defined as estimate divided by standard error, with an approximate standard normal null distribution [64]. All respondents were included in the network and data were corrected for respondents who entered the study at a later time point or left the study at an earlier time point [110]. Estimations were made using the Method of Moments, and for the Spanish schools, because of the small networks, by Maximum Likelihood [109] with the usual convergence criteria. When analyzing small datasets in combination with complex models, the Maximum Likelihood method is preferred above the Method of Moments as it tends to be a more efficient estimation method in the sense of producing estimates with smaller standard errors. When analyzing larger datasets the efficiency advantage is negligible and there is no reason not to use the less time-consuming Method of Moments [109].

Finally, for each country, results of all school network analyses were combined in a meta-analysis. The null hypotheses that in all schools a nonpositive effect (right one-sided test) is present, and that in all schools a nonnegative effect (left one-sided test) is present, were separately tested by Fisher's combination of one-sided tests [111]. To control for multiple (right and left) testing, there was deemed to be significant support for an effect if either of these combination tests was significant at level 0.025. The null hypothesis that effect parameters are constant across schools was tested by the method of Cochran adapted for network dynamics by Snijders and Baerveldt [106, 112].

The relative contribution of selection, influence, and alternative mechanisms to observed smoking behavior similarity

A descriptive statistic measuring the similarity of individuals linked in a network is Moran's I, a spatial autocorrelation coefficient [113] applied to adjacency in the network. By calculating the average network autocorrelation in networks simulated according to models with coefficients estimated under five different specifications, the contributions of each specification to observed smoking similarity between friends can be expressed, as described in [59]. For these analyses, only schools with a mean observed autocorrelation of at least 0.1 were included, thereby excluding five Danish and four Spanish schools. The first model specification was a baseline trend model based on the initially observed network and smoking data. The second model 
was a control model including all control variables but excluding smoking-based selection and influence of friendship networks. The third model was the second model to which all smoking-based selection effects were added, while the fourth model was the second model extended by all influence effects of friendship networks. Finally, the fifth model included all effects as reported in Table 2. Average network autocorrelations, based on a large number of simulations, are expected to be lowest in the first and second models, intermediate in the third and fourth, and highest in the fifth model. The relative increase, going from the second model to the third or fourth model, compared with the increase from the second model to the fifth, indicates the proportion of network autocorrelation that can be allocated to smoking-based selection or influence friendship networks respectively. Network autocorrelations were averaged across schools to obtain an overall conclusion per country.

\section{RESULTS}

\section{Friendship network changes: selection of friends}

The results of the first part of the model, examining effects on which friendship selection depended, are depicted in Table 3. In all six countries, adolescents who smoked more had a greater tendency to choose friends who likewise scored high on smoking behavior, as indicated by the significant interaction effect of smoking behavior adolescent $\times$ potential friend.

The results for the included alternative selection mechanisms revealed that adolescents significantly preferred not to select arbitrary friends, but preferred to have reciprocal friendships and to be friends with their friends' friends, as indicated by the significant negative outgoing friendships effects, and significant positive reciprocity and transitivity effects. Adolescents did not select friends based on similar alcohol consumption. However, only in Spain adolescents were more popular (more often selected as a friend) when they drank more alcohol, as indicated by the significant effect 'alcohol consumption of potential friend'. Friends were selected based on similar age in the Netherlands, Portugal, and Spain. In Denmark, the younger the adolescents were, the more they were selected as friends and selected friends themselves. Adolescents tended to select friends based on similar gender in all countries. Selection based on similar nationality was found only in the Netherlands, although non-native Portuguese adolescents selected fewer friends themselves but 
were more often selected as friends compared to native Portuguese adolescents. In the UK and Spain, adolescents selected friends based on similar school achievement.

\section{Smoking behavior changes: Influence processes}

The results of the smoking behavior evolution part of the model are reported in Table 4. Only in Finland and the Netherlands was adolescent's smoking behavior influenced by friends' smoking behavior. Being more often nominated as a friend (popularity) or nominating more friends did not have an impact on own smoking behavior as indicated by no significant effects due to incoming friendships and outgoing friendships.

In all countries, the significant effects of the control variables 'tendency to smoke' and 'addiction to smoke' implied that adolescents had an overall tendency not to smoke, but smoking behavior was self-reinforcing due to its addictive nature. In the Netherlands and Portugal, adolescents with at least one smoking parent had a higher tendency to smoke. In the Dutch sample, adolescents also smoked more when at least one of their siblings smoked. Drinking more alcohol resulted in a more smoking in the Netherlands and the UK. The tendency to smoke increased with age only in Portugal. In Portugal, the UK, and Spain, girls smoked more than boys. Nonnative Spanish adolescents (those adolescents with a South American, Mediterranean, Asian and other background) had a higher tendency to smoke compared to native Spanish adolescents. Adolescents with lower school achievement had a higher tendency to smoke in Denmark and Spain.

\section{The relative contribution of selection and influence processes to smoking behavior similarities}

The contribution of the various mechanisms generating similarities in smoking behavior among friends is depicted in Figure 2. The slices labeled 'selection' represent the proportion of similarity attributed to smoking-based friendship selection processes. The mean values of this proportion were 32\% in Finland, 19\% in Denmark, $45 \%$ in the Netherlands, $27 \%$ in Portugal, $17 \%$ in the UK, and $47 \%$ in Spain.

The slices labeled 'influence' reflect the proportion of similarity attributed to influence from adolescent's friendship networks (smoking behavior of friends, and numbers of given and received friendship nominations). These proportions were $19 \%$ in Finland, $10 \%$ in Denmark, $23 \%$ in the Netherlands, $13 \%$ in Portugal, $7 \%$ in the UK, and $6 \%$ in Spain. 
CHAPTER 4 | SOCIAL NETWORK ANALYSES IN SIX EUROPEAN COUNTRIES

All selection and influence mechanisms except for smoking-based selection and influence of friends are jointly represented by the slices labeled 'control'. These proportions ranged from $1 \%$ in Portugal to $38 \%$ in Spain. Finally, the slices labeled 'trend' cover the consequences of the similarity observed in the preceding wave. The proportions of smoking behavior similarity explainable from the previous wave range from $9 \%$ in Spain to $49 \%$ in Portugal.

In all countries the proportion of smoking behavior similarity explained by smoking-based selection was higher than the proportion due to influence of adolescents' friendship network. A rather large proportion was explained by alternative selection and influence processes, and general trends we controlled for. 


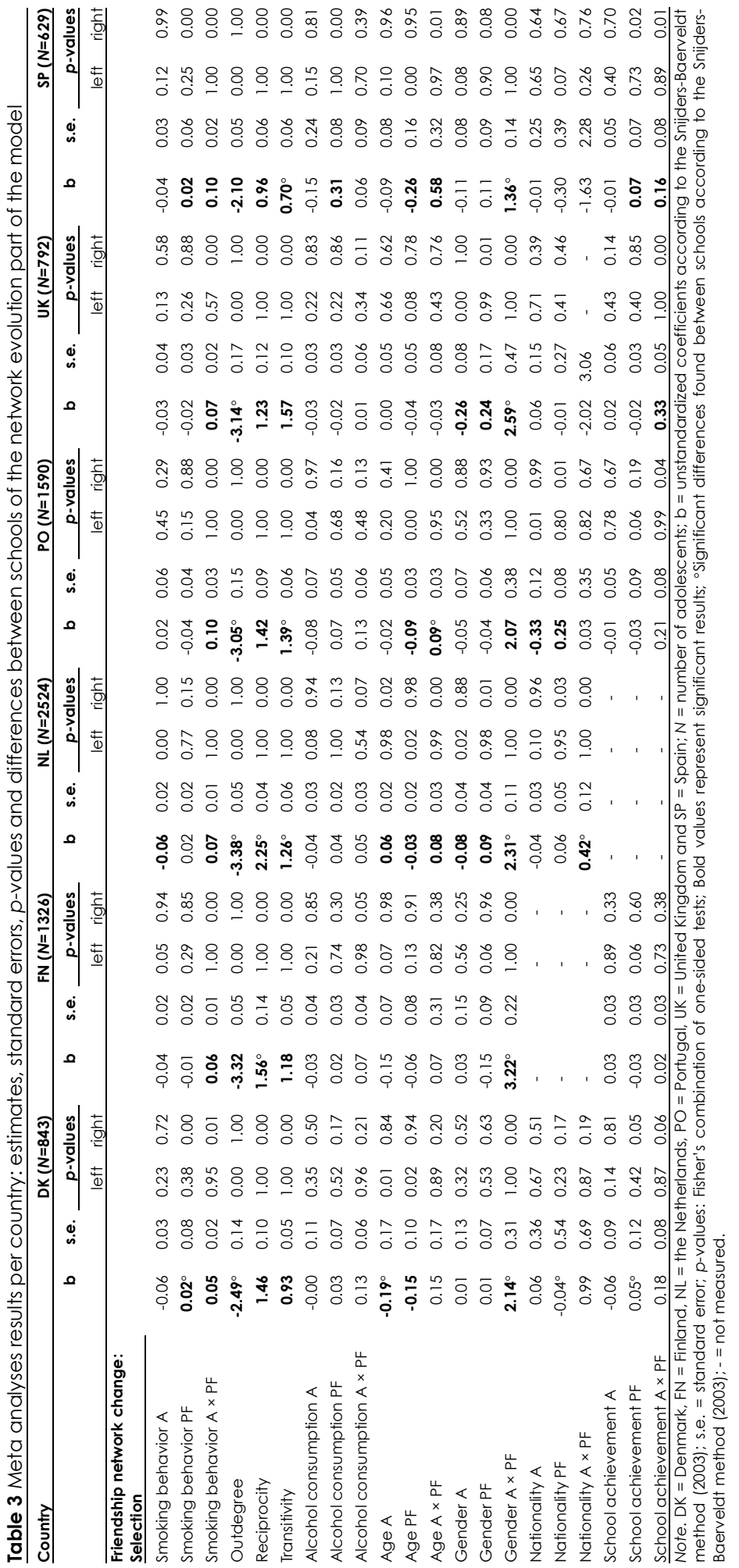




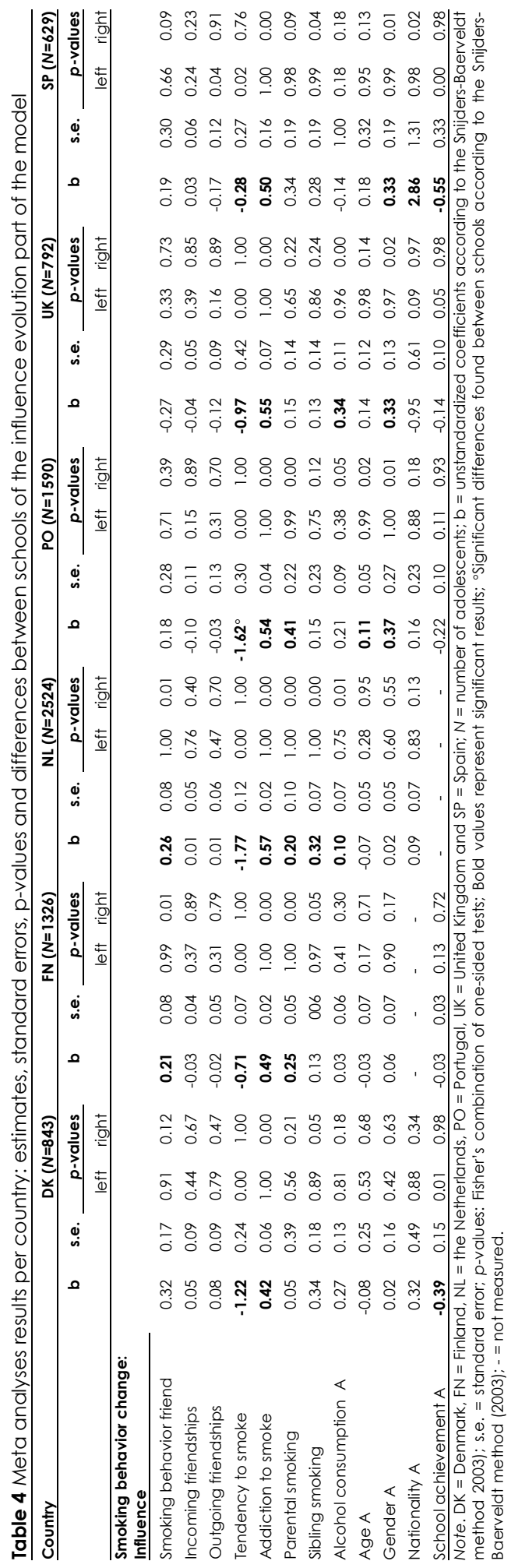


Figure 2 Therelative contribution of selection andinfluence processesto smoking behaviorsimilarities between friends

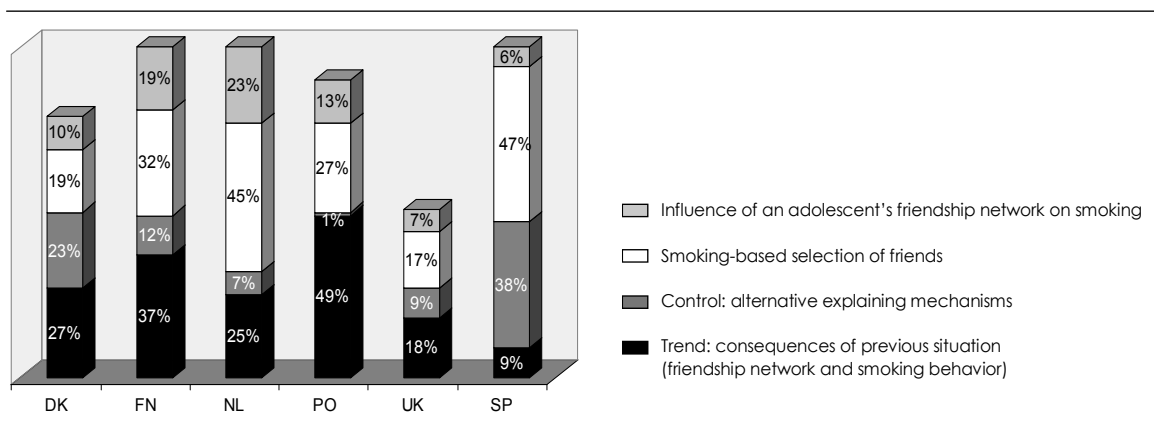

\section{DISCUSSION}

This study aimed to disentangle influence and selection by examining the coevolution of adolescents' friendship networks and smoking behavior in six European countries, using newly developed social network analysis techniques.

Our findings clearly demonstrate that selection processes play an important role in creating smoking behavior similarity within friendships. Adolescents preferred to select friends with similar smoking behavior in each country. These results support previous findings about the importance of selection processes [12, 13, 23, 29, 105].

One of the strengths of the present study was the inclusion of a number of alternative explaining mechanisms to counter a possible overestimation of selection based on similar smoking behavior. Adolescents highly preferred to reciprocate friendships and to become friends with friends of friends, which is in line with previous research [106, 107]. Gender similarity was another important selection criterion. These significant results as well as those of previous studies [52], underline the necessity to control for such mechanisms when examining selection and influence processes.

The most remarkable finding of this study may be that support for influence of friends' smoking behavior on adolescent smoking was only found in Finland and the Netherlands. In all countries selection based on smoking behavior was more important than peer influence in explaining similarity in smoking status between friends. Although often suggested [1 1, 100, 105], our results do not entirely confirm the importance of peer influence. Our results slightly differ from a previous study on similar data [29], in which no support was found for peer influence in Finland and the Netherlands, but was found in Portugal. In this study [29] friendship composition 
changes were not taken into account and smoking behavior of friends as reported by the adolescents instead of actual smoking behavior of friends was used, which may have led to biased estimations of influence and selection processes. Furthermore, the present study uses data over a longer time period, which yields higher power for discovering effects. In addition, the influence and selection processes may change over time. Further research is needed to examine this possibility.

As in previous studies [18, 75, 114], we found support for parental smoking influences on adolescent smoking in Finland, the Netherlands, and Portugal. Support for influence of sibling smoking was only found in the Netherlands. These results might imply that a substantial number of adolescents were not influenced by their direct social environment but choose to smoke for different reasons. We did not find evidence that being more often selected as a friend (being popular) or selecting more friends oneself affected adolescent smoking behavior in any of the six countries. This is in contradiction with [115] who reported that popular students were more likely to become smokers compared to less popular peers. This difference might be due to the different population or to the stronger control exerted in our study for alternative mechanisms.

\section{Limitations}

This study was subject to limitations. First, self-reported smoking behavior was not validated by biochemical measures. However, under optimized measurement conditions that assure anonymity, self-reports have been shown to be reliable and to correspond well with biological indicators [86, 116]. During the ESFA project, measurement conditions were optimized by guaranteeing strict confidentiality [66]. Second, no direct measures of parental and sibling smoking were available, which might have biased estimated parental and sibling smoking behavior effects. However, previous research has demonstrated that adolescents aged 13-17 years can be used as reliable sources to report on the smoking status of their parents [103]. Third, the use of a fixed name generator might have limited adolescents' possibilities to nominate their best friends. However, previous research, allowing $7^{\text {th }}$ graders to nominate any number of friends, showed that on average only 4.09 friends were nominated [88]. Allowing more than five nominations might provoke adolescents to nominate peers who are not 'best' friends. Fourth, we focused on friendships within the same school grade. Although these specific friends form an important social environment, they do not represent the entire social network of adolescents. Older 
friends may be an important source of influence, and as adolescents grow older themselves, friends are more likely to come from various settings. Future social network studies should aim to include all friends outside and inside school. Fifth, we could not include classroom membership effects because this information was not available, which is a disadvantage mainly for the countries where grades were large (Netherlands, Portugal, UK), as SIENA makes the assumption that all network members are equally available as potential friends. This may have biased the transitivity parameter and it might have led to an overestimation of smoking-based selection since adolescents in the same classroom may have a higher chance to become friends. Future research should include measurements of classroom membership and test this possibility. Finally, differences between countries and between schools within countries were not explored. Although we tested for differences across schools, we did not examine school level factors such as educational level and school size. It will be interesting to include such factors in future multilevel analyses.

\section{Practical implications}

This study has several practical implications. First, if adolescents are less strongly influenced by their friends to start smoking than assumed earlier, it is conceivable they choose to smoke due to earlier formed smoking-related attitudes. In these cases, smoking prevention programs will probably benefit more from reinforcing nonsmoking attitudes than from teaching adolescents to cope with social influences. Second, smoking prevention should not solely focus on social influence, but also consider selection processes. Previous research has already emphasized that peer network structure needs more attention within prevention programs besides the promotion of social influence skills $[90-92,116,117]$. Especially in countries where influence processes play a role, prevention could benefit from creating non-smoking majorities within groups, working with popular peers as role models, or increasing selfawareness regarding imitation and selection. Finally, the different results across countries regarding influence processes call for a multilevel approach which could reveal the effects of cultural norms [118], school size or classroom organization. Cultural norms may protect against smoking, but may also foster smoking as a normative behavior. Greater stability in classroom composition over years could promote friendship stability and decrease opportunities for selection as well as influence processes. 


\section{CHAPTER 5}

A social network analysis in a Finnish sample

Based on: Mercken, L., Snijders, T., Steglich, C., Vartiainen, E., \& De Vries, H. (2009) Dynamics of adolescent friendship networks and smoking behavior. Social Networks (In press). 


\section{ABSTRACT}

The mutual influence of smoking behavior and friendships in adolescence is studied. It is attempted to disentangle influence and selection processes in reciprocal and non-reciprocal friendships. An actor-based model is described for the co-evolution of friendship networks and smoking behavior. This model considers alternative selection and influence mechanisms, and models continuous-time changes in network and behavior. The data consists of a longitudinal sample of 1326 Finnish adolescents in 11 high schools. Findings suggest that selection as well as influence processes play an important role in adolescent smoking behavior. Selection had a relatively stronger role than influence, in particular when selecting non-reciprocal friends. The strength of both influence and selection processes decreased over time. 


\section{INTRODUCTION}

One of the main preventable causes of cancer, heart disease, and premature death is cigarette smoking [2, 119-121]. Many youngsters experiment with smoking, which often results in becoming a regular smoker in adulthood [3]. During adolescence, smoking behavior tends to be similar among friends [8-11]. This similarity in smoking behavior, which can be regarded as network autocorrelation [122], could be caused by selection of similar others as friends as well as by influence processes where friends adjust their smoking behavior to each other, or by a combination of these. This article will demonstrate the use of stochastic actor-based models [62, 123] capable of disentangling influence and selection processes by simultaneously representing changes in friendship network structure and changes in smoking behavior among adolescents. In particular, the impact of friendship reciprocity on selection and influence processes will be explored. Reciprocal friendships may offer higher friendship quality, which in turn could result in more opportunities for influence processes leading to smoking behavior similarities among friends [27, 43]. Pearson and Michell [40] examined non-reciprocal and reciprocal friendships and concluded that adolescents in the periphery of peer groups were the most important targets of influence.

Several studies attempted to disentangle selection and influence processes in the context of smoking behavior and suggested that selection of friends based on smoking behavior may be just as important as influence processes to explain similarity among friends, or even more important $[12,13,23,29,96,105]$. Studies that considered friendship reciprocity showed mixed results. Several studies found stronger support for influence within reciprocal compared to non-reciprocal friendships [27, $43,96]$, other researchers did find strong support for influence of non-reciprocal or desired friends [100].

Disentangling selection and influence processes, as well as the role of friendship reciprocity in these processes, is difficult, due to the dynamic interdependent nature of friendship networks and smoking behavior. Previous studies have three main shortcomings. First, although most previous studies did include important alternative influence processes such as the influence of parental and sibling smoking [18], they did not control adequately for alternative explanatory selection mechanisms. A smoking adolescent, for example, might choose a smoker as a friend because this individual already indicated the adolescent as his friend (reciprocity) or because this particular person was already a friend of the 
adolescent's other friends (transitivity). Further, the selection of this smoking friend might be based not on similarities in smoking behavior but on similarities in age, gender, alcohol consumption, school achievement, etc. Support for these alternative causes of tie formation was found by previous researchers [52, 106, 107]. Failing to control for alternative mechanisms might result in an overestimation of the strength of smoking-based selection processes. Second, researchers did not consider the continuous changes of network structure and smoking behavior over time happening between observations. Longitudinal data is mostly gathered at only a few discrete moments, which makes it impossible to unequivocally identify the processes responsible for a network or behavioral change. In between two observation moments, changes will occur in friendships and smoking behavior, and a change may even be followed by a change back to the original value before the next observation moment. Figure 1 demonstrates influence and smoking-based selection processes that are likely to be diagnosed incorrectly on the basis of discrete observations if change between the observations is not accounted for. Consecutive observations are denoted here by $T_{1}$ and $T_{2}$, and some sequences of changes that may have occurred between observations are also indicated. Analysis techniques that are based on classifying observed changes as being due to influence or selection without accounting for the possibility of other intervening changes [13, 29, 124] may be misleading, and it is preferable to use a technique that does take this possibility into account. Finally, independence assumptions that underlie the employed statistical methods are violated. Even more advanced statistical techniques such as structural equation modeling used for this type of data $[29,124]$ assume incorrectly that there are no dependencies caused by the network structure of an adolescent. For example, a given individual's value on smoking behavior could appear within more than one observation, e.g., as the smoking behavior dependent variable for one case, and as smoking behavior of one of the friends supplying data for the independent variables in other cases.

New social network analysis methods have recently been developed which are able to consider alternative explanatory selection mechanisms, to model continuous-time changes in smoking behavior and friendship networks, and to take dependencies into account caused by the network structure. Stochastic actorbased models $[64,65]$, have been developed to include network and behavior coevolution $[62,123]$. The following section will describe such an actor-based model for network-behavior co-evolution in the context of adolescent friendship networks and smoking behavior. A more extensive introduction is given in [125]. 
Figure 1 Possible changes between behavior and network observations

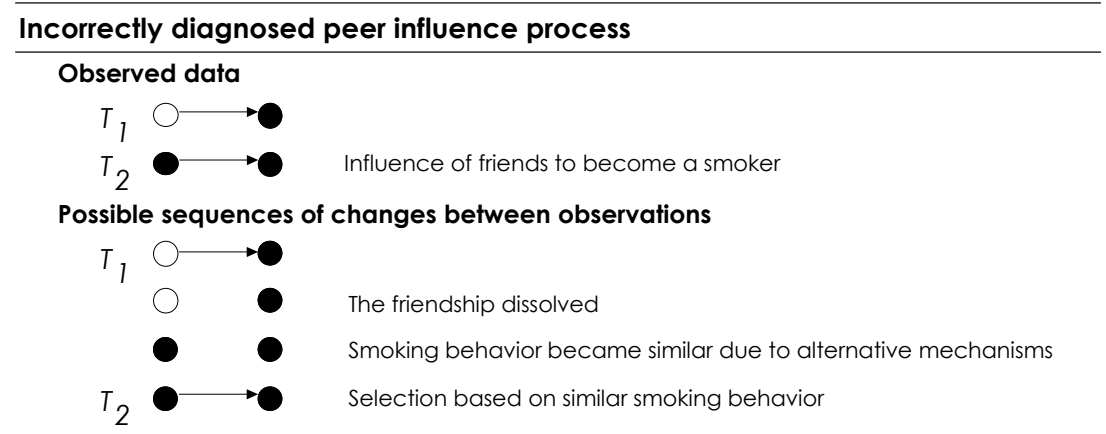

Incorrectly diagnosed smoking - based selection process

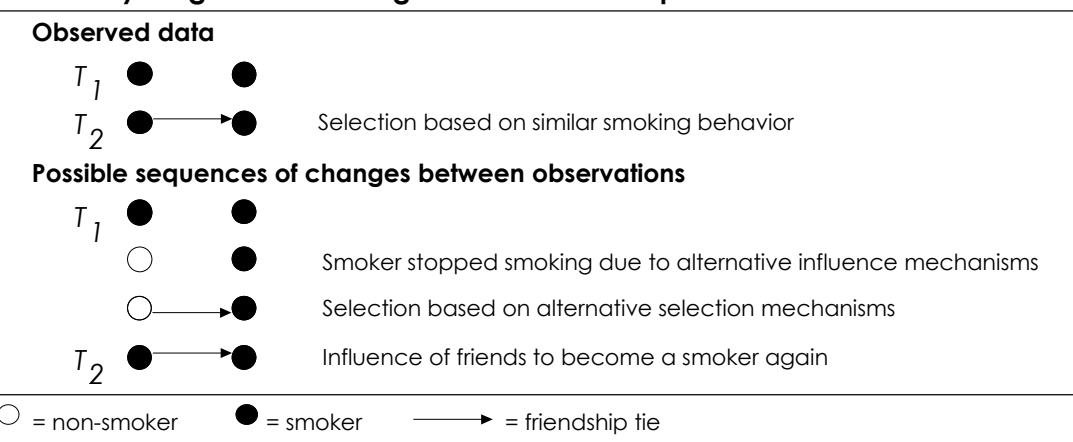




\section{An actor-based model for friendship network- smoking behavior co-evolution}

Actor-based models for network-behavior co-evolution assume that at two or more observation moments, a directed network and one or more behavioral variables are observed for a finite set of social actors. In our study, the actors are adolescents in a school. The network is a dichotomous relational variable, in our case indicating who directs friendship ties to whom. The behavior is assumed to be a dichotomous or discrete ordinal variable, in our case smoking behavior. Adolescents can change their smoking behavior, and also their friendship ties, in response to the current friendship network structure and the smoking behavior of the other adolescents in the network. It is assumed that all actors are fully informed about the state of the network, covariates and smoking behavior of all other actors in the network. Actors are only allowed to change their own outgoing ties and their own smoking behavior; they cannot make changes in outgoing ties or smoking behavior of other actors. Each adolescent is furthermore assumed to make decisions to change friendship ties or smoking behavior by probabilistic rules depending on the current configuration of network and behavior. The functions that determine the probabilities of change are called the objective functions, and there are separate objective functions for network change and for behavior change. Probabilities of change to a particular network or behavior state are higher accordingly as the objective function is higher; see Snijders, Steglich, and Schweinberger [62] and Snijders, van de Bunt and Steglich [125]. One interpretation is that the changes are the result of choices to optimize the actor's position in the network according to short-term preferences and constraints combined with random disturbances, and the objective function represents these short-term preferences and constraints. Finally, all actors consider and execute network and smoking behavior changes independently, given the current state of the network and everybody's behavior. Actors may change only one friendship tie or one level of smoking behavior at any moment in time. This implies that actors may react to each others' changes in friendship ties and smoking behavior, but do not negotiate or otherwise make joint changes based on a prior agreement. Therefore a negotiation like 'when you start smoking, l'll become your friend' would need to be represented as the result of two smaller changes, between which the causal link cannot be enforced: 'you may start smoking, but whether I will become your friend remains to be seen'. Note that while an actor cannot be certain that starting to smoke will result in a friendship, if smoking similarity has a positive effect on friendship selection then the actor does know that starting to smoke will increase the probability of being considered as a friend by smoking schoolmates. 
The actor-based models for co-evolving networks are modeled according to a continuous-time Markov process in which likely developmental trajectories between observation moments are imputed (continuous-time property), and changes adolescents make are assumed to depend only on the current state of affairs (Markov property).

To model the co-evolution of friendship and smoking two models are created, one for friendship network change and one for smoking behavior change. The network evolution and behavior evolution models are integrated as one internally dependent process. In this manner, the current state of the continuously changing friendship network can be a dynamic constraint for changes in smoking behavior, while simultaneously the current state of smoking behavior can act as a dynamic constraint for changes in the friendship network.

Using this approach, four main research questions are addressed in this study:

1. Do adolescents select friends based on similar smoking behavior?

2. Are adolescents influenced by friends to adjust to their smoking behavior?

3. Does the strength of these selection and influence processes differ for nonreciprocated and reciprocated friendships?

4. What is the relative contribution of selection and influence processes over time?

\section{METHODS}

\section{Participants}

The sample consists of 11 Finnish schools containing 1326 adolescents that participated as a control group in the ESFA study [66, 76], which was an intervention study with interventions taking place at the community level. The participating Finnish organization demanded that participating schools be located exclusively in Helsinki. In this research region, communities/neighborhoods were randomly selected. High schools within the target communities were asked to participate, indicating that they would have a $50 \%$ chance of becoming an experimental school. Experimental schools were excluded in this study since the intervention may have changed the relationship between variables of interest. The present study included all control schools that participated at each of the four measurement times, resulting in 11 schools with in total 1326 participating adolescents. 


\section{Procedure}

Self-administered questionnaires were distributed in schools among seventh graders during autumn 1998, since smoking onset is most likely to occur among this age group. Follow-up was conducted respectively 12, 24, and 30 months later [66, 76]. On the days of data collection, present students were asked to complete the questionnaire. It was explained to the adolescents that responses would be treated confidentially and they could refuse to participate. Questionnaires were returned in sealed envelopes to guarantee their anonymity. In Finland, the overall rate of refusals to participate was $3 \%$.

\section{Questionnaire}

Friendship ties were assessed by one question in which adolescents could name up to five best friends inside and/or outside school [81]. Only best friends inside school in the same grade are included here, to have complete networks as data material.

Smoking behavior of adolescents was assessed by one question: "On average, how many cigarettes do you smoke during a week (also count the weekend)?" $(0=0,1=$ between 0 and 1, $2=2-10,3=11-30,4=$ more than 30$)$.

Parental smoking behavior was measured by two questions: 'Does your father (male caregiver) smoke?' and 'Does your mother (female caregiver) smoke?', and was recoded into one variable $10=$ neither smokes, $1=$ at least one parent (caregiver) smokes).

Sibling smoking behavior was measured by two questions: 'Do one or more of your brother(s) smoke?' and 'Do one or more of your sister(s) smoke?', and was recoded into one variable ( $0=$ no siblings smoke, $1=$ at least one sibling smokes).

Alcohol consumption $(0=0$ glasses of alcoholic drinks per week, $1=1$ or 2 glasses, 2 = 3 to 5 glasses, $3=$ more than 5 glasses), age (in years), gender $(0=$ boy, 1 $=$ girl), and self-reported school achievement ( $1=$ lower third of the class, $2=$ middle third, $3=$ highest third) were also recorded. 


\section{Plan of analysis}

\section{Model development}

An actor-based model was constructed which consisted of two models: one model simulates the evolution of the friendship network (which allows the study of selection processes), and the other model simulates the evolution of smoking behavior (for studying influence processes). The combined model simulates selection and influence processes simultaneously while controlling either process for the other one. Four observations were available for each school. None of the respondents were excluded from the network. Even those respondents who entered the study at a later time point or left the study before the end of the study were included in the model for the duration of their membership of the school [110]. The detailed mathematical specification of actor-based models is given by Snijders, Steglich, and Schweinberger [62] and Snijders, van de Bunt and Steglich [125]. Table 1 presents a list of descriptions and mathematical specifications of all the included effects and a sketch of the model is as follows.

The friendship network evolution part of the model specifies the preferred direction of network change by a list of functions of network 'effects', depending on current network structure as well as on attributes of adolescents. Friendship choice probabilities depend on these effects. This list contains four smoking-related friendship selection components: the effect of adolescent's smoking behavior on number of friends chosen (smoking adolescent), the effects of smoking behavior of potential friends and its square on choosing them (smoking potential friend, squared smoking potential friend), and an interaction effect between smoking adolescent and smoking potential friend, which is used to test that adolescents who smoke more also prefer friends who smoke more (smoking behavior adolescent $x$ potential friend). The reason for including both the raw and the squared value of the behavior of friends is to control for possible nonlinearities and to have a more robust assessment of the influence of friends on the respondent [125]. As network dynamics have major endogenous components $[52,64,108]$, several characteristics of the current network and various individual attributes were included as control variables. These were: number of friends chosen (outgoing friendships), number of reciprocal friends chosen (reciprocity), and the number of friends who are also a friend-of-a-friend (transitivity). Furthermore, as control effects, similarity on alcohol consumption, age, gender, and school achievement were included, as well as the effects of these attributes on the number of friends chosen (e.g., age adolescent) and on the propensity to be chosen 
as a friend (e.g., age potential friend). The friendship network evolution part of the combined model is summarized in the upper part of Table 1. The rather large number of control effects is necessary because friendship selection is a multidimensional process and smoking behavior may correlate with many other attributes as well as with network position, so that the failure to control adequately for such characteristics could lead to misleading inferences.

The smoking behavior evolution part of the model likewise specifies preferred directions of changes in smoking status. It encompasses a list of functions of network, smoking behavior, and other attributes on which probabilities of changes in smoking behavior may depend. This list contained one main friendship-related influence component: the effect of smoking behavior of friends on adolescent smoking behavior. Included control effects were the basic tendency to smoke expressed as a quadratic function of smoking behavior and therefore represented by two parameters, one for the linear and one for the quadratic term; smoking behavior of parents and siblings; and own alcohol consumption, age, gender, and school achievement. This list is given as the lower part of Table 1.

Since previous social network analyses within six European countries [126] did not find evidence for the effects of number of received and outgoing friendship nominations on smoking behavior, these extra effects on which smoking behavior changes might depend were only tested by means of score tests [127]. Similarly, two interaction effects of smoking-based selection and influence of friends with reciprocity were tested by score tests to examine whether the strength of smokingbased selection and influence of friends differs between non-reciprocated and reciprocated friendships. Because of co-linearity considerations, this score test procedure for identifying additional effects to include in a model is generally preferable to attempts of direct estimation of the effects in question. The latter procedure runs the risk of not obtaining convergence in the estimation algorithm, and not being able to identify the newly included effect, and possibly others.

\section{Statistical analysis}

For each school separately, the combined model, including the four score tests, was analyzed using the Unconditional Method of Moments $[62,64]$ in SIENA version 3.14 (Simulation Investigation for Empirical Network Analysis) [109]. The included effects were tested on the basis of t-ratios defined as estimate divided by standard error, with an approximate standard normal null distribution [64]. Subsequently, the results of all separate school network analyses were combined in a meta-analysis. The t- 
ratios were combined separately for each of the effects in Table 1. It was desired to use a combination method with a good power to detect various patterns of non-zero parameter values across the 11 schools and with a minimum of assumptions. For each effect, the overall null hypothesis that the corresponding parameter is 0 in all schools was tested by using Fisher's combination procedure [1 111] twice, once for a right-sided and once for a left-sided test. In the right-sided test the null hypotheses is that in all schools the coefficient of this effect is non-positive, and the alternative hypothesis is that in at least one school the coefficient is positive. In the left-sided test, the same is done with interchanged roles of 'positive' and 'negative'. The test statistic in Fisher's procedure is minus twice the sum of the natural logarithms of the $p$ values of the one-sided tests for the individual schools, with under the combined null hypothesis a chi-squared distribution having, for 11 schools, 22 degrees of freedom. To control for multiple (right and left) testing, there was deemed to be significant support for an effect if either of these combination tests was significant at level 0.025 (Bonferroni correction). In addition, the null hypothesis that effect parameters are constant across schools was tested by the method of Cochran (1954) adapted for network dynamics by Snijders and Baerveldt [106, 112]. Depending on the results of the score tests, the final model for analysis was extended by the effects for which the score test produced evidence.

Additional models will be estimated to explore whether smoking-based selection and influence effects differ between the three separate data waves. Network autocorrelations will be examined to explore the relative contribution of selection, influence, and alternative mechanisms to observed smoking behavior similarities among friends. 


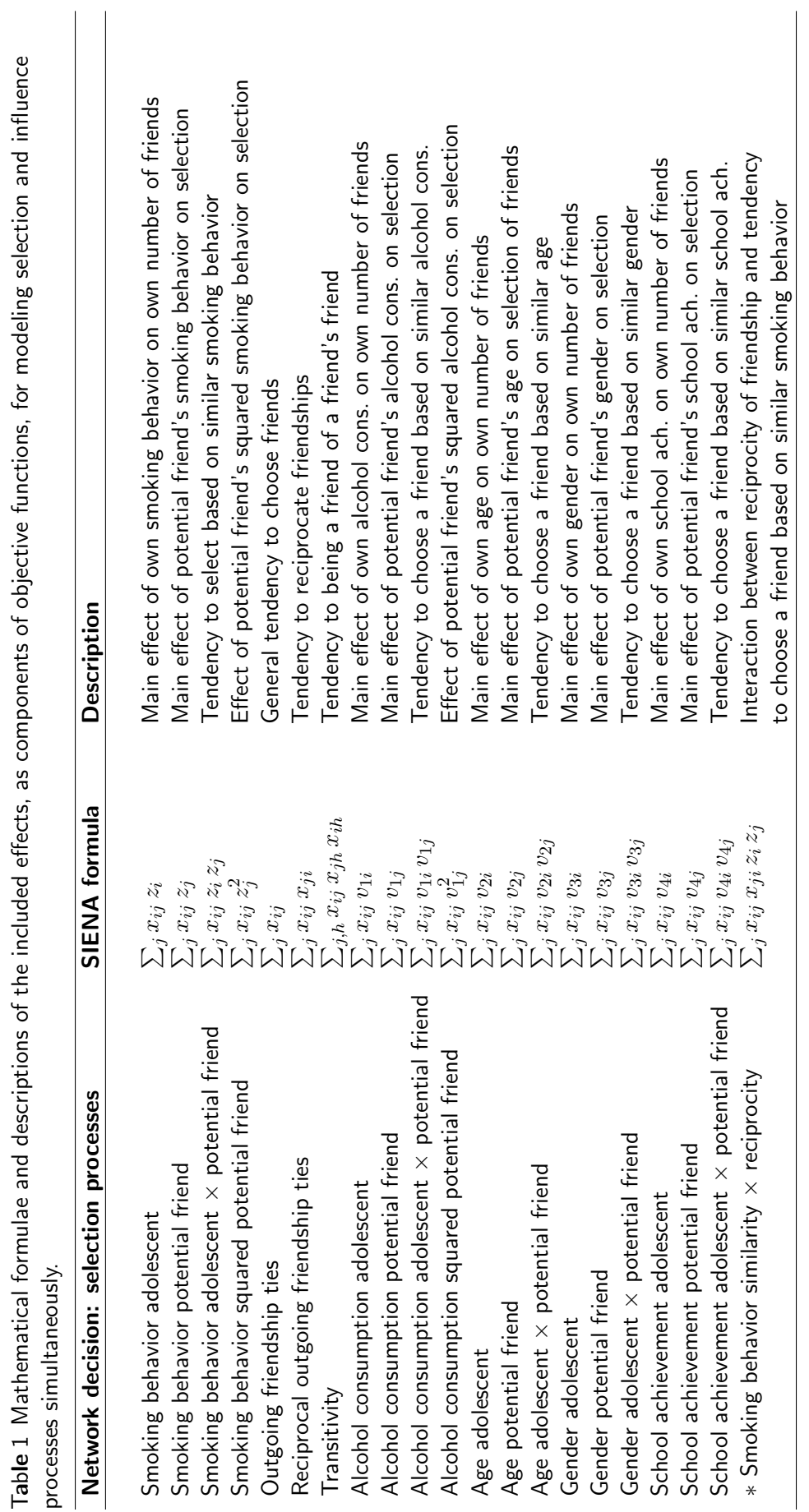




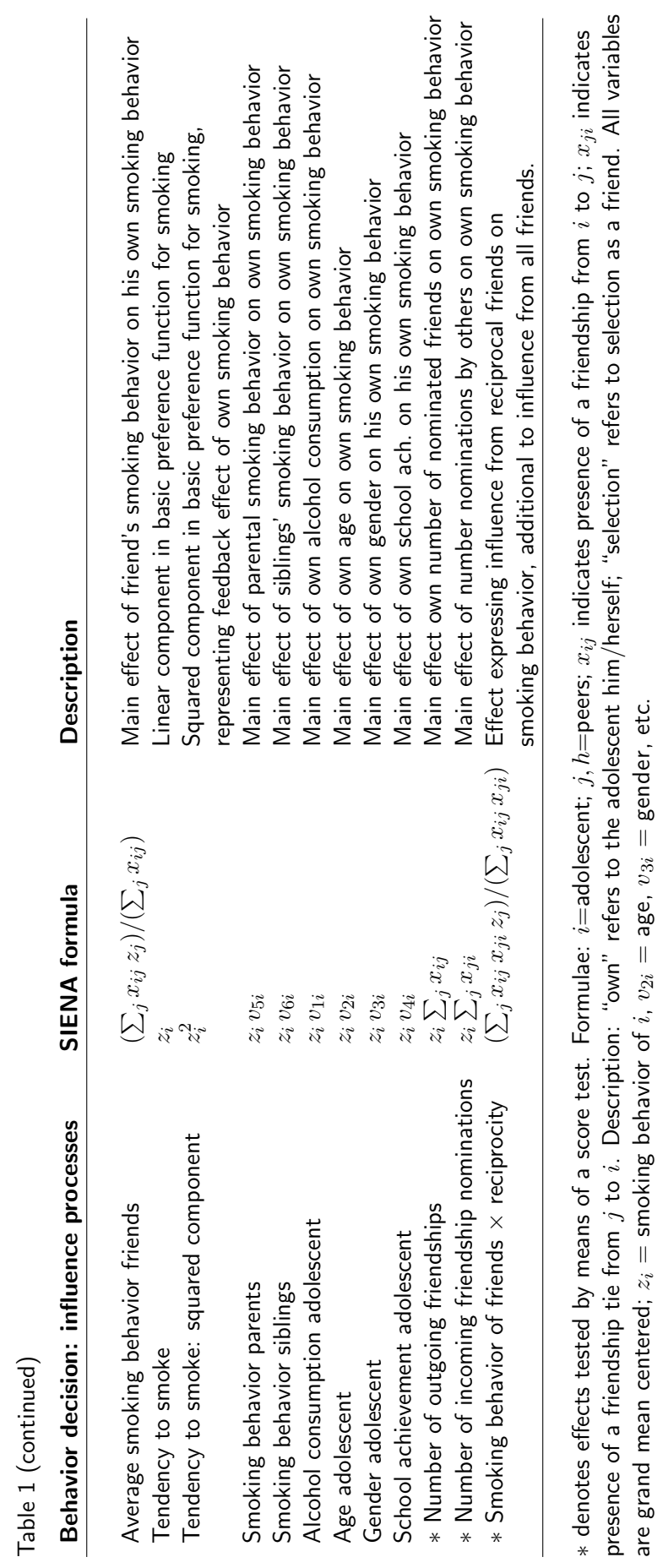




\section{RESULTS}

\section{Descriptive statistics}

Table 2 demonstrates the average number of friends per adolescent, smoking behavior of adolescents, and observed network autocorrelations for each wave, and baseline demographic characteristics. The average numbers of friends generally increased between subsequent waves, with one exception (slight decrease after wave 2), while smoking behavior increased over time. The observed network autocorrelation slightly decreased from 0.42 in the first wave to 0.39 in the last wave.

Table 2 Descriptive statistics of network structure and individual characteristics

\begin{tabular}{|c|c|c|c|c|}
\hline & Wave 1 & Wave 2 & Wave3 & Wave 4 \\
\hline Average number of outgoing ties & 1.67 & 2.02 & 1.75 & 1.77 \\
\hline Average smoking behavior adolescent & 0.46 & 0.90 & 1.36 & 1.38 \\
\hline \multirow[t]{2}{*}{ Average observed network autocorrelation (Moran's I) } & 0.42 & 0.42 & 0.41 & 0.39 \\
\hline & Period 1 & Period 2 & Period 3 & \\
\hline Average number of adolescents joining a school network & 8 & 9 & 2 & \\
\hline \multirow[t]{2}{*}{ Average number of adolescents leaving a school network } & 8 & 10 & 20 & \\
\hline & Mean & & & \\
\hline Number of adolescents in school & 121 & & & \\
\hline Alcohol consumption adolescent & 0.3 & & & \\
\hline Percentage at least one smoking parent & 49.9 & & & \\
\hline Percentage at least one smoking sibling & 23.0 & & & \\
\hline Age (in years) at baseline & 13.4 & & & \\
\hline Percentage females & 47.0 & & & \\
\hline School achievement & 2.0 & & & \\
\hline
\end{tabular}

\section{Friendship network evolution}

The score test for interaction between friendship reciprocation and selection based on similar smoking behavior was significant (left one-sided Fisher combination, Chisquare $=39.6, d f=20, p=0.006)$ and therefore this effect was included in the final model.

Results for the friendship network evolution part of the model are reported in the upper part of Table 3. In discussing these we focus on the effects linking friendship and smoking. Adolescents who smoked more had a greater tendency to choose friends who likewise scored high on smoking behavior as indicated by the significant interaction smoking ego by smoking alter effect (Chi-square $=70.8, \mathrm{df}=22, \mathrm{p}<$ 
0.001). There were no significant main effects of the smoking behavior of the adolescent or the potential friend on friendship selection.

The additionally included significant interaction effect with reciprocity furthermore indicated that the tendency to choose similar smoking friends (interaction smoking ego by smoking alter), was significantly higher when selecting non-reciprocated friends as compared to reciprocal friends. The remaining effect of selection of reciprocated friends based on similar smoking behavior was calculated by combining the results of the interaction smoking ego by alter effect and the three way interaction of smoking ego by alter by reciprocity effect for each school separately. The results for all schools were then examined using the Fisher combination of one-sided tests (left-sided chi-square $=24.94$, $\mathrm{df}=18, \mathrm{p}=0.13$; rightsided chi-square $=13.20$, $d f=18, p=0.78)$. These results imply that similarity of smoking behavior played a significant role in friendship selection only when there was not already a tie in the reciprocal direction.

Results for other effects included in the friendship dynamics model and considered here as control effects, are in line with what has been found more often in research on friendship dynamics and are not specifically discussed here. To have a more detailed representation of the contributions of smoking of the adolescent and the potential friend on tie formation and dissolution, the upper part of Table 4 gives the total contribution of all smoking-related effects to the objective function for network change, as defined on the basis of estimated average parameters. This technique, explained in Section 16 of Snijders et al. (2007), represents numerically the attractiveness of friends with specific smoking behavior values to be chosen as a friend by the adolescent. The most attractive potential friends for adolescents that smoke less than 1 cigarette each week are the classmates who do not smoke at all. For adolescents who smoke 1 or more cigarettes each week, the most attractive friends are those who smoke at the highest rate. Furthermore, smoking behavior of potential friends mattered most in friendship selection for adolescents who smoked at the highest rate.

\section{Smoking behavior evolution}

The score tests for the interaction between friendship reciprocation and influence of friends' smoking behavior, the number of outgoing friendship nominations and the number of received friendship nominations, all turned out not to be significant and were therefore not included in the final model. 
The results of the smoking behavior evolution part of the social network analysis, which specifies the preferred direction of change in smoking behavior, are reported in the lower part of Table 3. Adolescent's smoking behavior was influenced by the smoking behavior of their friends. Adolescents adjusted their smoking behavior to the smoking behavior of their friends (Chi-square $=42.7$, $\mathrm{df}=22, \mathrm{p}=0.005$ ). The lower panel of Table 4 gives the combined contribution of the smoking behavior of the adolescent and that of his/her friends' to the objective function for smoking. This represents the attractiveness of different values of smoking behavior, depending on the behavior of the friends. The most attractive smoking behaviors for adolescents lie on both ends of the spectrum (the highest and lowest scores). Smoking in-between the highest and lowest rate, for example score 2, is very volatile and will be absorbed to one of the ends of the spectrum. The most attractive smoking behavior for adolescents with friends that do not smoke is non-smoking. For adolescents whose friends do smoke, the most attractive behavior seemed to be smoking at the highest rate.

With regard to the other included effects, the significant squared component of the smoking tendency expresses the positive feedback effect of current smoking, confirming the addictive nature of smoking. Further, adolescents having at least one smoking parent or sibling had a higher tendency to smoke.

\section{Additional analyses}

The results of the additional analyses indicated no differences in the main included smoking-based selection and influence effects between the three separate waves. Adolescents selected friends based on similarities in smoking behavior during each wave. The score test included to test for differences in selection based on similar smoking behavior between non-reciprocal and reciprocal friends, was significant in the second wave (left-sided chi-square $=37.99, \mathrm{df}=22, \mathrm{p}=0.02$; right-sided chisquare $=8.18, \mathrm{df}=22, p=1.00$ ) implying that the tendency to choose similar smoking friends was significantly higher when selecting non-reciprocated friends as compared to reciprocal friends. This significant result was not found in the first (left-sided chisquare $=31.86, \mathrm{df}=20, \mathrm{p}=0.05 ;$ right-sided chi-square $=15.13, \mathrm{df}=20, \mathrm{p}=0.77$ ) and third wave (left-sided chi-square $=17.07, \mathrm{df}=22, p=0.76$; right-sided chi-square $=$ $20.76, d f=22, p=0.54)$. In none of the three separate waves, a significant effect of average smoking behavior of friends was found, although a trend was visible. 
Table 3 Results meta analysis Finland

\begin{tabular}{|c|c|c|c|c|c|c|}
\hline & \multirow{2}{*}{\multicolumn{2}{|c|}{$\begin{array}{c}\text { Snijders-Baerveldt } \\
\text { Method }\end{array}$}} & \multicolumn{4}{|c|}{ Fisher combination test } \\
\hline & & & \multicolumn{2}{|c|}{ Left one-sided } & \multicolumn{2}{|c|}{ Right one-sided } \\
\hline & b & s.e. & $X^{2}$ & $p$ & $X^{2}$ & $p$ \\
\hline \multicolumn{7}{|l|}{ Network decision: Selection processes } \\
\hline Smoking behavior adolescent & -0.035 & 0.029 & 30.8 & 0.10 & 13.2 & 0.93 \\
\hline Smoking behavior potential friend & -0.106 & 0.197 & 18.6 & 0.67 & 16.0 & 0.82 \\
\hline $\begin{array}{l}\text { Smoking behavior adolescent } \\
\mathrm{x} \text { potential friend }\end{array}$ & $0.093^{* * *}$ & 0.016 & 3.7 & 1.00 & 70.8 & $<0.001$ \\
\hline $\begin{array}{l}\text { Smoking behavior adolescent } \\
\times \text { potential friend } \mathrm{x} \text { reciprocity }\end{array}$ & $-0.126 * *$ & 0.040 & 40.3 & 0.002 & 5.4 & 1.00 \\
\hline Smoking behavior squared potential friend & 0.049 & 0.102 & 16.6 & 0.79 & 18.2 & 0.70 \\
\hline Outgoing friendship ties & $-3.356^{* * *}$ & 0.224 & 276.2 & $<0.001$ & 0.6 & 1.00 \\
\hline Reciprocal outgoing friendship ties & $1.763^{* * * \circ}$ & 0.088 & 0.9 & 1.00 & 3031.9 & $<0.001$ \\
\hline Transitivity & $1.175^{* * *}$ & 0.052 & 0.1 & 1.00 & 3104.0 & $<0.001$ \\
\hline Alcohol consumption adolescent & -0.023 & 0.047 & 24.1 & 0.34 & 14.5 & 0.88 \\
\hline Alcohol consumption potential friend & $0.189 *$ & 0.081 & 10.5 & 0.98 & 40.0 & 0.01 \\
\hline $\begin{array}{l}\text { Alcohol consumption adolescent } \\
\times \text { potential friend }\end{array}$ & $0.092^{*}$ & 0.048 & 9.4 & 0.99 & 34.6 & 0.04 \\
\hline Alcohol consumption squared potential friend & $-0.100 *$ & 0.040 & 38.3 & 0.02 & 9.4 & 0.99 \\
\hline Age adolescent & -0.148 & 0.080 & 30.5 & 0.11 & 10.8 & 0.98 \\
\hline Age potential friend & -0.037 & 0.052 & 30.4 & 0.11 & 12.7 & 0.94 \\
\hline Age adolescent $x$ potential friend & 0.063 & 0.133 & 14.0 & 0.83 & 19.5 & 0.49 \\
\hline Gender adolescent & 0.019 & 0.130 & 21.2 & 0.51 & 26.9 & 0.22 \\
\hline Gender potential friend & -0.117 & 0.088 & 31.1 & 0.09 & 13.0 & 0.94 \\
\hline Gender adolescent $x$ potential friend & $3.188^{* * * \circ}$ & 0.231 & 0.0 & 1.00 & 382.9 & $<0.001$ \\
\hline School achievement adolescent & 0.018 & 0.033 & 14.8 & 0.87 & 22.5 & 0.43 \\
\hline School achievement potential friend & -0.034 & 0.031 & 31.4 & 0.09 & 19.0 & 0.64 \\
\hline $\begin{array}{l}\text { School achievement adolescent } \\
\times \text { potential friend }\end{array}$ & 0.023 & 0.036 & 17.0 & 0.77 & 23.4 & 0.38 \\
\hline \multicolumn{7}{|l|}{ Behavior decision: Influence processes } \\
\hline Average smoking behavior friend & $0.221^{* *}$ & 0.069 & 7.6 & 1.00 & 42.7 & 0.005 \\
\hline Tendency to smoke & $-0.922^{* * * \circ}$ & 0.081 & 8477.3 & $<0.001$ & 0.0 & 1.00 \\
\hline Tendency to smoke: squared component & $0.484^{* * *}$ & 0.022 & 0.1 & 1.00 & 3112.4 & $<0.001$ \\
\hline Smoking behavior parents & $0.232^{* * *}$ & 0.047 & 2.2 & 1.00 & 67.2 & $<0.001$ \\
\hline Smoking behavior siblings & $0.125^{*}$ & 0.054 & 11.4 & 0.97 & 34.4 & 0.04 \\
\hline Alcohol consumption adolescent & 0.009 & 0.053 & 24.0 & 0.35 & 22.0 & 0.46 \\
\hline Age adolescent & 0.029 & 0.064 & 21.9 & 0.47 & 21.8 & 0.47 \\
\hline Gender adolescent & 0.056 & 0.049 & 15.9 & 0.82 & 28.4 & 0.16 \\
\hline School achievement adolescent & -0.023 & 0.041 & 28.6 & 0.16 & 19.9 & 0.59 \\
\hline
\end{tabular}


Table 4 The attractiveness of smoking behavior

Selection

\begin{tabular}{|c|c|c|c|c|c|}
\hline & 0 & 1 & 2 & 3 & 4 \\
\hline 0 & 0.288 & 0.038 & -0.114 & -0.169 & -0.125 \\
\hline 1 & 0.159 & 0.002 & -0.058 & -0.019 & 0.118 \\
\hline 2 & 0.030 & -0.034 & -0.001 & 0.131 & 0.361 \\
\hline 3 & -0.099 & -0.070 & 0.056 & 0.281 & 0.604 \\
\hline 4 & -0.228 & -0.107 & 0.113 & 0.431 & 0.847 \\
\hline
\end{tabular}

Influence

friend

\begin{tabular}{|c|c|c|c|c|c|c|}
\hline \multirow{6}{*}{ adolescent } & & 0 & 1 & 2 & 3 & 4 \\
\hline & 0 & 1.654 & 1.430 & 1.207 & 0.983 & 0.760 \\
\hline & 1 & 0.013 & 0.011 & 0.008 & 0.006 & 0.003 \\
\hline & 2 & -0.659 & -0.441 & -0.222 & -0.004 & 0.214 \\
\hline & 3 & -0.364 & 0.075 & 0.515 & 0.954 & 1.394 \\
\hline & 4 & 0.899 & 1.560 & 2.220 & 2.881 & 3.541 \\
\hline
\end{tabular}

Contributions of smoking behavior of adolescent and potential friend to the objective function for friendship (top panel) and for smoking (bottom panel). Smoking behavior is coded: $0=0$ cigarettes each week; $1=$ between 0 and $1 ; 2=1-10 ; 3=11-30 ; 4=>30$

\section{Network autocorrelations}

A descriptive statistic measuring the similarity of individuals linked in a network is Moran's I, a spatial autocorrelation coefficient [113] applied to adjacency in the network rather than in space. By calculating the average network autocorrelation in simulated models with coefficients estimated under various model specifications, it is possible to express the contributions of each of these specifications to observed smoking similarity between friends. For this purpose we follow the approach explained more fully in Steglich, Snijders and Pearson (2009). Five model specifications were used. The first is a baseline model expressing only the effects of the initial smoking distribution within the initial network, the time trends in number of friends and in smoking. This is a straw man model which serves only as a baseline. The second is a model including all control effects but excluding smoking-based friendship selection and excluding influence between friends. In this model the network dynamics and the smoking dynamics are modelled in a realistic way, but these two processes are assumed not to influence one another. The third model differs from the second by the inclusion of smoking-based selection effects, while the fourth differs from the second by including influence by the smoking behavior of friends. These two models, therefore, represent friendship-smoking co-evolution with 
only smoking-based friendship selection, and only influence from friends on smoking behavior, linking the two sub-processes. The fifth model, finally, was obtained as the end result of the analysis presented in Table 3, assuming smoking-based selection as well as influence by friends. Network autocorrelation as expressed by the average Moran's I in a large number of simulations is expected to be lowest in the first and second models, intermediate in the third and fourth, and highest in the fifth model. The relative increase when going from the second to the third or from the second to the fourth model, compared with the increase from the second to the fifth, indicates the proportion of network autocorrelation that can be attributed to selection or to influence, respectively. Network autocorrelations were averaged across schools to obtain an overall picture for each of the three waves.

Figure 2 presents the allocation of network autocorrelation as observed in waves 2-4 to diverse mechanisms generating similarity of friends, estimated by comparing the average values of Moran's I obtained under the five presented models. The slices labeled 'selection' cover the five included effects of adolescent's smoking behavior on network change (adolescent's own smoking behavior, effect of smoking behavior of potential friends, squared smoking behavior of adolescent, interaction between smoking behavior of adolescents and potential friends, and interaction of the latter effect with reciprocity), and the slices labeled 'influence' the single included effect of an adolescent's network on his own smoking behavior (smoking behavior of nominated friends). The slices labeled 'trend' cover the consequences of the network autocorrelation observed in the preceding wave, such as would be generated by a very simple model including only basic trends and the tendency to select arbitrary friends. The other alternative explanatory mechanisms, such as reciprocity, transitivity and influences of covariates, are represented by the slices labeled 'control'. The small 'ambiguous' slice is the proportion of network autocorrelation that could be allocated either to influence or to selection, depending on the order in which these components are included in the model.

The proportion of network autocorrelation allocated to smoking-based friendship selection was higher than the proportion allocated to influence processes in each of the three waves. The mean proportion allocated to selection decreased from $46 \%$ during the first wave to $31 \%$ during the third wave. The mean proportions allocated to influence decreased from $22 \%$ during the first wave to $15 \%$ during the last wave. The increasing proportion allocated to trend effects reflects that patterns of smoking behavior and friendships crystallize over the years of adolescence: the effect of the preceding wave becomes more and more important. Other selection 
processes, such as selecting friends who are friends of friends or selection based on gender, and other influence processes, such as from parents and siblings, also play an important role to explain the observed network autocorrelations.

Figure 2 The relative contribution of selection and influence processes to smoking behavior similarities

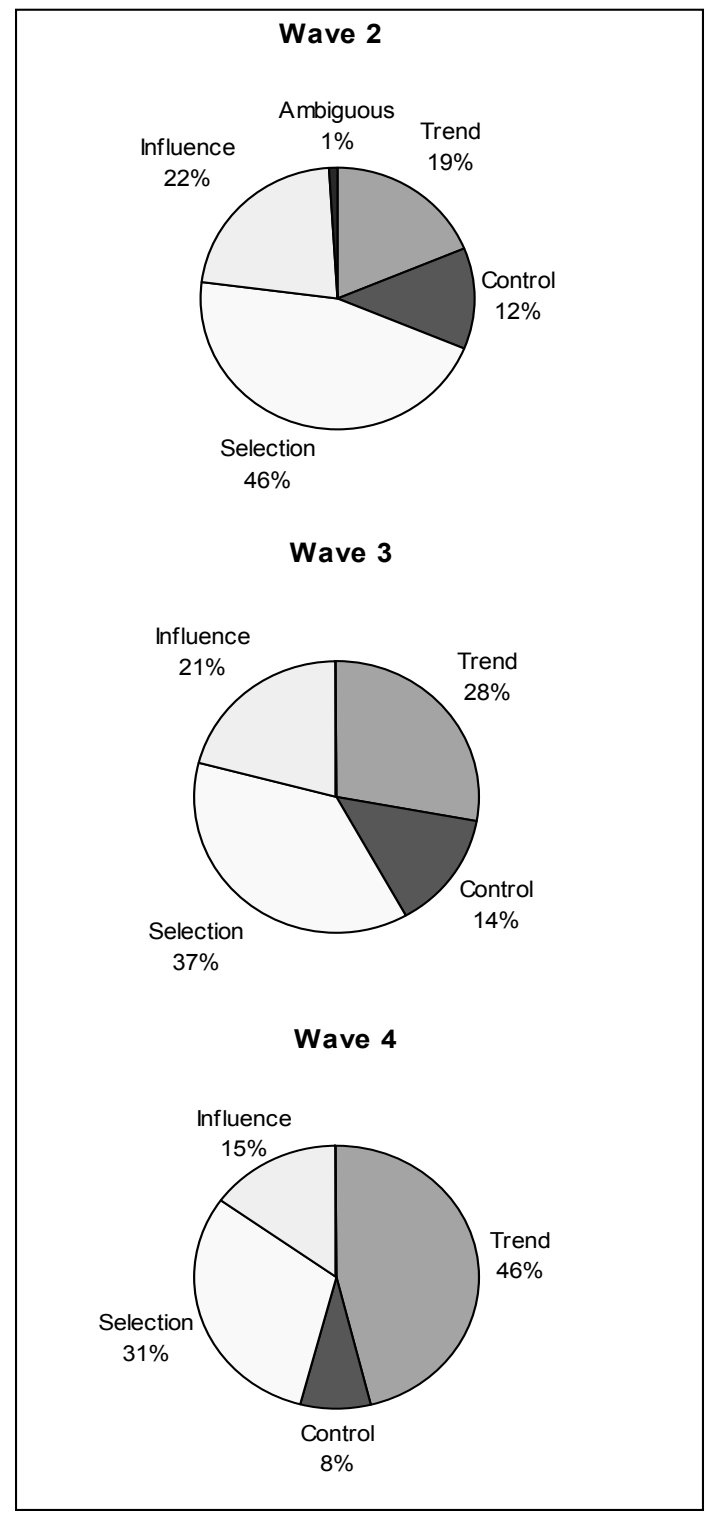




\section{DISCUSSION}

The main goal of the present study was to test social influence and social selection processes in the interdependent dynamics of adolescent friendship networks and smoking behavior, while controlling the test of each of these processes for the other process and for other processes in the dynamics of friendship and of smoking. This was studied on the basis of a 4-wave panel study of smoking dynamics among adolescents (age 13-16 years) in 11 schools in Finland. Due to the dynamic and interdependent nature of friendship network ties and adolescent smoking behavior, a recently developed social network analysis method was employed: an actorbased model for friendship network and smoking behavior co-evolution. This method can account for alternative mechanisms explaining selection, as well as dependencies caused by network structure, and is capable of modeling continuoustime changes in smoking behavior and friendship networks.

Our findings demonstrate that selection and influence processes both played an important role in creating and maintaining smoking behavior similarity within friendships. In line with previous research that already acknowledged the importance of selection processes $[12,13,23,29,105]$, adolescents preferred to select friends with similar smoking behavior. Non-smokers were the most attractive for those smoking less than once a week, whereas those smoking on average more than one cigarette per week preferred to choose friends that smoked at the highest rate.

Support for peer influence within friendships, which is often suggested in the literature $[11,100,105]$, was also found. The results of this analysis differ from the results of a previous study on the Finnish ESFA data [29], in which no support was found for influence among friends. However, in this previous study [29] only data of the first two observations were included and other statistical methods were used which do not fully account for the dependencies caused by the network structure, potentially leading to biased estimations of peer influence.

We furthermore found that the strength of influence processes did not differ between non-reciprocated and reciprocated friendships, implying that adolescents were influenced equally by their non-reciprocal and reciprocal friends. This contradicts previous studies that argue that reciprocal friendships encompass more opportunities for influence processes to cause smoking behavior similarities among friends due to higher friendship quality [27, 43].

The importance of smoking for selection of friends did differ between reciprocated and non-reciprocated friendships. Results indicated that similarity of 
smoking behavior played a role in friendship selection mainly for the selection of nonreciprocated friends. Furthermore, this effect was mainly found to be significant in the second data wave according to the results of additional analyses in the three separate data waves. Selection of non-reciprocated friends might be regarded as an initial phase of friendship formation, while returning a friendship might be regarded as a later phase of the friendship formation process. Previous research already demonstrated that similarity appeared to be relatively more important during the initial phases of friendship formation, when individuals choose potential friends. In later phases, when they establish long-lasting friendships, the provision of social and emotional resources such as companionship, emotional support, help and self-validation, becomes more important [46], and may allow for more dissimilarity in smoking behavior. Another explanation might be the social position of the nonreciprocal friends in a larger friendship group. Previous studies have demonstrated that adolescents in the periphery of their peer group were the most important targets for selection [40]. Future research should examine selection and influence processes considering friendship reciprocity, in the context of larger friendship groups.

The present study included a number of alternative explaining mechanisms to counter biased estimations of influence and selection processes. In line with previous studies, this showed that adolescents highly preferred to reciprocate friendships and to become friends with friends of their friends [106, 107], and to select friends based on gender and alcohol consumption similarities $[52,89]$. This also yielded support for the feedback effect of current smoking behavior, which reflects the addictive nature of smoking. In line with previous studies [18, 75, 114], adolescents were furthermore influenced by the smoking behavior of their parents and siblings.

Smoking-based selection explained a larger proportion of smoking behavior similarity between friends, compared to influence of friends during each of the three successive waves. The contributions of both of these processes to similarity between friends of smoking behavior decreased over time while the contribution of the previous wave increased, reflecting that the friendship network and the smoking patterns become less fluid as adolescents age from 13 to 16 .

\section{Limitations}

The following limitations of this study may be mentioned. First, self-reported smoking behavior was not validated by biochemical measures. However, self-reports have been shown to be reliable and to correspond well with biological indicators when measurements are done under optimized measurement conditions, assuring 
anonymity [86]. During the ESFA project, measurement conditions were optimized by guaranteeing strict confidentiality of adolescent responses [66]. Second, the use of a name generator limited to a maximum of five friends might have limited adolescents' possibilities to nominate all their best friends. However, previous research, allowing $7^{\text {th }}$ graders to nominate any number of friends, showed that on average only 4.09 friends were nominated [88]. Allowing to nominate more than five best friends might provoke adolescents to nominate peers who are not 'best' friends. Third, we focused on friendships within schools in the same grade. Although for adolescents, these specific friends form an important social environment, they do not represent their entire social network of peers. Future social network studies should aim to include all friends outside and inside school. Fourth, standardized effect sizes are very hard to define and therefore not yet provided for complex models such as actor-based models which makes it difficult to compare our findings with results of previous studies. However, allocation of network autocorrelation to diverse processes (Figure 2) and calculating the attractiveness of friends (Table 4) is a good alternative way to demonstrate the strength of selection and influence processes. Fifth, in the present study, we controlled for alternative selection and influence mechanisms involving observed and reported variables, although there could be selection and influence mechanisms involving unobserved covariates too. Finally, the conclusions obtained here are based on the specification of the actor-based model described above, and it is possible that other specifications, e.g., controlling for other processes by including other characteristics of adolescents or different specifications of the influence mechanism, would yield different results.

\section{Practical implications}

This study has several practical implications. First, smoking prevention programs should not solely focus on social influence processes, but also consider peer selection processes. Previous research already emphasized that peer network structure needs more attention within prevention programs besides the promotion of social influence skills [90-92]. Second, the actor-based model for network and behavior co-evolution presented in the present study might be used also in different research fields to address a variety of interesting research questions. This model provides a tool to disentangle selection and influence mechanisms in numerous social network configurations and behaviors. Third, adolescents in the present study significantly selected friends based on similar gender, which indicates that there are gender segregated social networks of boys and girls. Future research might examine differences in selection and influence processes between boy and girl friendship network. 



\section{CHAPTER 6}

Selection and influence in gender segregated networks

Based on: Mercken, L., Snijders, T., Steglich, C., Vartiainen, E., \& De Vries, H. (2009) Selection and influence processes in gender segregated friendship networks (submitted for publication). 


\section{ABSTRACT}

Aims: The main goal of this study was to examine differences between adolescent male and female friendship networks regarding smoking-based selection and influence processes using newly developed social network analysis methods that allow the current state of continuously changing friendship networks to act as a dynamic constraint for changes in smoking behavior, while simultaneously allowing current smoking behavior to be a dynamic constraint for changes in friendship networks. Design: Longitudinal design with four measurements. Setting: Nine junior high schools in Finland. Participants: 1163 adolescents that participated in the control group of the ESFA (European Smoking prevention Framework Approach) study, including 605 adolescent males and 558 females. Measurements: Smoking behavior of adolescents, parents, siblings, and friendship ties. Findings: Smoking-based selection of friends was found in male as well as female networks. However, support for influence among friends was only found in female networks. Furthermore, females and males were both influenced by parental smoking behavior. Conclusions: Prevention programs need to focus on the role of selection processes besides paying attention to influence processes. Our results suggest that while females need to learn to cope with peer influences, both males and females may benefit from reinforcing non-smoking attitudes in order to increase the likelihood that they select non-smoking peers. Prevention programs should furthermore be combined with smoking cessation interventions for parents. 


\section{INTRODUCTION}

Cigarette smoking continues to be one of the main preventable causes of cancer, heart disease, and premature death in many countries [2, 119-121]. During adolescence many youngsters experiment with smoking which may lead to regular smoking [3].

Numerous studies report smoking behavior to be similar among friends [8-11]. Although early research predominantly argued that this similarity was caused by peer influence, recent studies have provided evidence that similarity is also caused by selection of similar friends $[12,13,23,26,29,75,96,128]$. Friendship networks are an important aspect of an adolescent's life. Children tend to associate mainly with same-sex others and most close friends are of the same gender [51]. Although friendships between males and females begin to develop during adolescence, gender similarity among friends is still marked $[52,53]$. Same-gender peer relationships are an important socializing context that influences the development of gender differences in social interaction styles. Differences between female-female friendships and male-male friendships may lead to differences in smoking-based selection and influence processes during adolescence. This study aims to examine selection and influence processes, differentiated by gender, in the context of smoking behavior among adolescent friendship networks.

Researchers have demonstrated that friendship patterns differ for males and females. Females seem to have more intimate friendships [51, 54, 55], report higher intimacy levels [55], and are more likely to turn to peers for support [57], which could result in female networks offering more opportunities for influence. Previous research furthermore has found that females perceived more social pressure to smoke [129, 130] and were more susceptible to social influences [131]. However, to our knowledge, no study specifically examined differences between adolescent male and female friendship networks regarding smoking-based selection and influence processes.

The present study will examine selection and influence processes in male and female networks by using newly developed methods of social network analysis [62, 89]. These new methods, in contrast to conventional statistical methods previously used to disentangle selection and influence processes, allow the current state of the continuously changing friendship networks to act as a dynamic constraint for changes in adolescent smoking behavior, while simultaneously allowing the current 
state of smoking behavior to be a dynamic constraint for changes in friendship networks. Possible unobserved changes in friendships and smoking behavior that may occur in between two measurement moments can be taken into account, and, besides including other possible important determinants of smoking behavior such as age, parental and sibling smoking behavior [18], these methods allow controlling for other determinants of friendship selection besides smoking-based selection of friends. Some of these determinants are reciprocation of friendship; to become a friend of somebody who already is a friend of a friend [106, 107]; and selection based on alcohol consumption [89], age, ethnicity and education [52].

We hypothesize that smoking-based selection will be present in males and females and that females will be more susceptible to influence than males. We will furthermore explore the impact of reciprocity of friendship on the strength of smoking-based selection and influence processes.

\section{METHODS}

\section{Participants}

The sample consisted of 1163 Finnish adolescents that participated as a control group in the ESFA study [66, 76]. Participating schools were based on a random selection of communities in the Helsinki region and each had a $50 \%$ chance of becoming an experimental school. The present study included all control schools that participated at each of the four measurements and encompassed a minimum number of 20 males as well as females, resulting in 9 schools including 605 males and 558 females.

\section{Procedure}

Self-administered questionnaires were distributed among all 7th graders of the participating schools. Follow-up was conducted 12, 24 and 30 months later [66, 76], similar questionnaires were distributed among $8^{\text {th }}$ graders (12 month follow-up) and $9^{\text {th }}$ graders (24 and 30 months follow-up). All students present on the days of data collection completed the questionnaire. It was explained that responses would be treated confidentially. Students could refuse to participate, and all students returned their questionnaires in sealed envelopes to guarantee anonymity. At baseline, the proportion of refusals was .03\% [66]. 


\section{Questionnaire}

The ESFA questionnaire was based on earlier studies about adolescent smoking behavior [76-80, 132].

Friendship ties were assessed by one question in which adolescents could name up to five best friends inside and/or outside school [81]. Only best friends inside school in the same grade are included, as only they also filled out the questionnaire.

Smoking behavior of adolescents was assessed by one question: 'On average, how many cigarettes do you smoke during a week (also count the weekend)?' $(0=0,1=$ between 0 and 1, $2=2-10,3=11-30,4=>30)$. Note that this measure will be used to model smoking behavior of ego and alters in the network analysis.

Parental smoking behavior was measured by two questions: 'Does your father (male caregiver) smoke?' and 'Does your mother (female caregiver) smoke?', and was recoded into one variable ( 0 = neither smokes, 1 = at least one smokes).

Sibling smoking behavior was measured by two questions: 'Do one or more of your brother(s) smoke?' and 'Do one or more of your sister(s) smoke?', and was recoded into one variable $(0=$ no siblings smoke, 1 = at least one smokes).

School achievement was assessed by one question: 'Last year, how well did you do in school, compared to the others in your class?' ( $1=$ among the lower third of my class, $2=$ the middle third, $3=$ the best third).

Alcohol consumption ( $0=0$ glasses of alcoholic drinks per week, $1=1$ or 2 glasses, $2=3$ to 5 glasses, 3 = more than 5 glasses), and age (in years), were also recorded.

\section{Analysis plan}

A stochastic actor-based model [59, 62, 64, 65] was constructed to realistically represent the mutual dependencies between friendship formation and dissolution on the one hand, and change in smoking behavior on the other hand, by a simulation model. This model takes into account the mutual feedback processes between friendship and smoking occurring between the observations. This new approach was applied successfully earlier to delinquency [107] and alcohol consumption [133]. All respondents were included and the model accounted for some respondents entering the study later or leaving earlier [109]. Missing values on adolescents' attributes and smoking behavior were allowed and treated as non-informative in the estimation procedure and imputed by a mean value for the start of the simulations. The model encompasses two parts: one part models friendship network changes 
(selection processes), the other part models smoking behavior changes (influence processes). Both parts are integrated as one internally dependent process. In this manner, selection and influence processes can be examined simultaneously while controlling either process for the other one. Mathematical specifications are given by $[62,64,127]$ and a sketch of the model is described in the next section.

\section{Friendship network changes: selection processes}

The friendship network evolution part of the model specifies the preferred direction of friendship change by including a number of effects that determine probabilities of changes in friendship status, such as current network structure and adolescent's attributes. This list contained four main smoking-based friendship selection components: the effect of adolescent's smoking behavior on number of friends chosen (smoking behavior ego); the effect of potential friends' smoking behavior (raw and squared value) on choosing them (smoking behavior alter, smoking behavior squared alter); and the interaction between smoking behaviors of adolescents and potential friends, which allows to test that adolescents who smoke more also prefer friends who smoke more (smoking behavior ego $\times$ alter). We included the raw and the squared value of alters' smoking behavior to control for possible curvilinear dependence of the attractiveness of potential friends on their smoking behavior. Since friendship choices have been shown to depend strongly upon characteristics of the current network $[52,64,108]$, the effects of number of friends chosen (outdegree), number of reciprocal friends chosen (reciprocity), and number of friends chosen who are also a friend-of-a-friend (transitivity) were included. The selection model controlled for alcohol consumption, age, and school achievement of adolescents and potential friends. A complete overview of all included effects is presented in the upper part of Table 1.

\section{Smoking behavior changes: influence processes}

The smoking behavior evolution part of the model specifies the preferred direction of change in smoking behavior by including a list of functions of network, smoking behavior, and other attributes on which changes in smoking behavior may depend. The included effects are described in the lower part of Table 1. The list contains three main friendship network-related influence components: the effect of the average smoking behavior of friends on adolescent smoking behavior, the effect of number of received friendship nominations (incoming friendships), and the effect of number of outgoing friendship nominations on adolescent smoking behavior loutgoing 
friendships). Included control effects were the tendency to smoke, a feedback effect of own previous smoking behavior to control for nonlinearities in smoking behavior (tendency to smoke squared), parental and sibling smoking behavior, and adolescents' alcohol consumption, age, and school achievement.

Table 1 Included effects for modeling selection and influence processes

\begin{tabular}{l} 
Network decision: \\
Selection processes \\
\hline Smoking behavior ego \\
Smoking behavior alter \\
Smoking behavior alter squared \\
Smoking behavior ego $\times$ alter \\
Outdegree \\
Reciprocity \\
Transitivity* \\
Alcohol consumption ego \\
Alcohol consumption alter \\
Alcohol consumption alter squared \\
Alcohol consumption ego $\times$ alter \\
Age ego \\
Age alter \\
Age ego $\times$ alter \\
School achievement ego \\
School achievement alter \\
School achievement ego $\times$ alter \\
Extra effect tested with score test: \\
Smoking behavior \\
ego $\times$ alter $\times$ reciprocity
\end{tabular}

\section{Description}

Effect of the adolescent's own smoking behavior on selection of friends

Effect of potential friends' smoking behavior on selection of friends

Effect of potential friends' squared smoking behavior on selection of friends

Tendency to choose a friend based on similar smoking behavior

General tendency to choose a friend

Tendency to have reciprocal friendships

Tendency to become a friend of a friends' friend

Effect of the adolescent's own alcohol consumption on selection of friends

Effect of potential friends' alcohol consumption on selection of friends

Effect of potential friends' squared alcohol consumption on selection of friends

Tendency to choose a friend based on similar alcohol consumption

Effect of the adolescent's own age on selection of friends

Effect of potential friends' age on selection of friends

Tendency to choose a friend based on similar age

Effect of the adolescent's own school achievement on selection of friends

Effect of potential friends' school achievement on selection of friends

Tendency to choose a friend based on similar school achievement

Effect to test whether selection based on similar smoking behavior differs when selecting a reciprocal or non-reciprocal friends

\section{Behavior decision:}

Influence processes

Smoking behavior friends **

Incoming friendships

Outgoing friendships

Tendency to smoke

Tendency to smoke squared

Smoking behavior parents

Smoking behavior siblings

Alcohol consumption adolescent

Age adolescent

School achievement adolescent

\section{Description}

Effect of friend's smoking behavior on his own smoking behavior

Effect of number of nomination by others on adolescent smoking behavior Effect of adolescents' number of nominated friends on own smoking behavior

General tendency to smoke

Feedback effect of adolescent's own smoking behavior on itself

Effect of parental smoking behavior on own smoking behavior

Effect of siblings' smoking behavior on own smoking behavior

Effect of an adolescent's alcohol consumption on own smoking behavior

Effect of an adolescent's age on own smoking behavior

Effect of an adolescent's school achievement on own smoking behavior

Extra effect tested with score test:

Smoking behavior

friends $\times$ reciprocity

*transitive ties; ** average alter effect

Effect to test whether the effect of friend's smoking behavior differs among reciprocal and non-reciprocal friends

Note Adequately controlling for attributes, such as age, results in a larger number of effects included in the friendship evolution part compared to the smoking behavior evolution part. This difference is due to the multidimensional nature of selection processes. The probability to select a friend may depend on the age of the adolescent, the age of the potential friend, and similarities in age of both. The effect of age on adolescent smoking behavior can be modeled by including only the effect of adolescents' age on their own smoking behavior. 


\section{Analysis}

For each wave, two separate friendship networks, a female network and a male network, were constructed within each participating school. All female adolescents within the school grade would be members of the female network; all male adolescents would be members of the male network. Since the focus of the present paper was on same-gender friendships, cross-gender friendships were excluded. For each male and female school network, the dynamic actor-based model was analyzed using SIENA (Simulation Investigation for Empirical Network Analysis) software [109]. The included effects were tested on the basis of $t$-ratios defined as estimate divided by standard error, which follow an approximate standard normal distribution [64]. Subsequently, results of all separate school network analyses were combined for males and females in two meta-analyses. The null hypothesis that the effect is 0 in all male or female networks was tested twice by Fisher's combination procedure [111], once for the right-sided test and once for the left-sided test. The right-sided test examines the null hypothesis that in all school networks the coefficient of this effect is nonpositive. The alternative hypothesis is that in at least one school the coefficient is positive. The left-sided test examines the null hypothesis that in all school networks the coefficient of this effect is nonnegative. The alternative hypothesis is that in at least one school the coefficient is negative. The Fisher test statistic is minus twice the sum of the natural logarithms of the p-values of the one-sided tests, with a chi-square distribution under the combined null hypothesis, having 18 degrees of freedom for 9 school networks. To control for multiple (right and left) testing, there was deemed to be significant support for an effect if either of these combination tests were significant at level 0.025. This Fisher's combination procedure [111], which will also be used to combine the T-tests for differences between males and females, is preferred over the Snijders- Baerveldt method [106] for meta-analysis as it does not make the assumption that estimated standard errors and estimated parameter values are uncorrelated, nor the assumption that the networks are a sample of a population.

The null hypothesis that effect parameters are constant across schools was tested by the method of Cochran [112] adapted for network dynamics by Snijders and Baerveldt [106, 112]. 


\section{Differences between reciprocal and non-reciprocal friendships}

To explore whether the strength of smoking-based selection of friends differs when selecting a non-reciprocal or reciprocal friend, and whether influence of friends differs within non-reciprocated and reciprocated friendships, interaction effects of smoking-based selection and influence of friends with reciprocity were tested by means of score tests [107, 127].

\section{The relative contribution of smoking-based selection and influence}

As a similarity measure of individuals linked in a network we used Moran's I, a spatial autocorrelation coefficient [113]. By calculating the average similarity of linked individuals in simulated models with coefficients estimated under different model specifications, the relative contributions of selection, influence, and control effects to observed smoking similarity between friends can be expressed. This method is explained in detail elsewhere [59]. Two male networks were excluded ( $N=92$ ) due to very low smoking rates (mean smoking behavior below 0.5 ) which might bias the results of these simulations. For comparative purpose the two female networks within these schools were also excluded $(\mathrm{N}=123)$. We shall graphically represent the average proportions of similarity allocated to smoking-based selection, peer influence, selection and influence mechanisms not based on links between friendship and smoking (i.e., controls), and general trend effects (inertia, effects of previous friendships and previous smoking behavior).

\section{RESULTS}

\section{Descriptives}

Table 2 presents the average network structure within the male and female school networks, the average smoking behavior in each wave, and baseline characteristics. Females nominated on average more friends than males. Females smoked at a slightly higher rate, and reported more often to have smoking parents and siblings.

\section{Differences between male and female networks: Selection processes}

The results for the friendship network evolution sub-model are reported in the upper part of Table 3. Males and females tended to nominate more smoking friends when their own smoking behavior was higher, as indicated by the significant 'smoking behavior ego $\times$ alter' effects. There were no significant main effects of adolescent 
own smoking behavior nor of smoking behavior of potential friends on friendship selection.

Results for the control effects indicated that males and females tended to choose relatively few friends (outdegree) and tended to reciprocate friendship choices (reciprocity). Females did have a significant higher tendency to become and remain friends with friends of their friends (transitivity). Adolescents did not select friends based on similar alcohol consumption, age, or school achievement. Among males, a propensity to select low achieving friends was found, while females tended to select friends drinking alcohol at a medium level (preferred scale value 1). None of the effects significantly differed between males and females except for the transitivity effect (combination of left-sided tests Chi-squared $=34.46$, d.f. $=18, p=$ 0.01). Females showed a stronger tendency to select friends of their friends compared to males.

\section{Differences between male and female networks: Influence processes}

The results of the smoking behavior evolution part of the model are reported in the lower part of Table 3. Females were influenced by the smoking behavior of their friends. Although there was evidence that the magnitude of effect of friend's smoking behavior differed across the nine included female school networks (Chisquare $=25.43$, d.f. $=8, p=0.001$, estimated true $S D=0.000$ ), the effect was found to be consistently positive (right-sided $p=0.002$, left-sided $p=0.58$ ). Although males and females did not differ significantly, males did not significantly adjust their smoking behavior to the smoking behavior of their friends (right-sided $p$-value $=.07$ ). However, there was evidence for some variation in the effects across the male networks (Chisquare $=16.33$, d.f. $=8, p=0.038$, estimated true $S D=0.000$ ). For both males and females the number of outgoing friendship nominations significantly influenced smoking behavior. Males and females who nominated fewer friends, tended to smoke more.

The control effects indicate that adolescents had a significant overall tendency not to smoke, but smoking behavior tended to be self-reinforcing as indicated by the significantly positive 'smoking tendency squared' effect. Male and female adolescents smoked more when at least one of their parents smoked and when they drank less alcohol. Females significantly smoked more when they were low achievers. High achieving males had a higher tendency to smoke but this effect was not significant. None of these effects significantly differed between males and females. 


\section{Differences between reciprocal and non-reciprocal friendships}

The score test of the interaction between smoking behavior of ego, alters, and reciprocity, indicated that in male and female networks, the tendency to select reciprocal or non-reciprocal friends who are similar in smoking behavior did not differ (combination left-sided tests chi-squared males $=20.53$, d.f. $=18, p=0.30$, females $=$ 29.07, d.f. $=18, p=0.05$; combination right-sided tests chi-squared males $=11.54$, d.f. $=18, p=0.87$, females $=12.77$, d.f. $=18, p=0.81$ ).

The score test of the interaction between friends' smoking behavior and reciprocity in its effect on smoking dynamics showed that among males as well as females influence of friends did not differ between reciprocal and non-reciprocal friendships (combination left-sided tests chi-squared males=13.34, d.f. $=18, p=0.77$, females $=7.72$, d.f. $=18, p=0.98$; combination right-sided tests chi-squared males=23.86, d.f. $=18, p=0.16$, females $=22.39$, d.f. $=18, p=0.22$ ). 
Table 2 Descriptive statistics of network structure and individual characteristics

MALES

FEMALES

Average network structure within schools

Average number of adolescents

Average number of friends

$\begin{array}{lll}\text { wave 1 } & 1.42 & 1.75 \\ \text { wave } 2 & 1.85 & 2.45 \\ \text { wave 3 } & 2.02 & 2.61 \\ \text { wave 4 } & 2.33 & 2.85\end{array}$

Reciprocity fraction

wave 1

wave 2

wave 3

wave 4

Transitivity index

wave 1

wave 2

$\begin{array}{ll}0.16 & 0.34\end{array}$

wave 3

wave 4

Moran's I network autocorrelation index

wave 1

wave 2

wave 3

wave 4

Individual characteristics

Mean smoking behavior adolescent

$\begin{array}{lc}\text { wave } 1 & 0.35 \\ \text { wave } 2 & 0.74 \\ \text { wave } 3 & 1.35 \\ \text { wave } 4 & 1.37 \\ \text { hol consumption adolescent } & 0.31 \\ \text { centage at least one smoking parent } & \\ \text { an age baseline (in years) } & 13.6\end{array}$

\begin{tabular}{rr}
0.35 & 0.47 \\
0.74 & 0.99 \\
1.35 & 1.37 \\
1.37 & 1.46 \\
0.31 & 0.25 \\
46 & 54 \\
20 & 27 \\
13.6 & 13.6 \\
1.94 & 2.01 \\
\hline
\end{tabular}

\begin{tabular}{lrrrr}
\hline Friendship ties & Wave 1 & Wave 2 & Wave 3 & Wave 4 \\
\hline \% friendship ties between males & 43.22 & 37.43 & 32.01 & 31.32 \\
\% friendships ties between females & 54.97 & 58.78 & 62.90 & 63.48 \\
\% excluded cross-gender friendship ties & 1.81 & 3.78 & 5.09 & 5.20 \\
\hline Smoking behavior is coded: 0 = 0 cigarettes each week; 1 = between 0 and 1;2=2-10; 3=11- 30; $=$ \\
>30; Alcohol consumption is coded: 0 = 0 glasses alcohol each week; 1 = 1 - 2; 2= 3- 5; 3=>5; School \\
achievement is coded: 1 = among the lower third of the class; 2 = middle third; 3 = best third.
\end{tabular}


CHAPTER 6 | SELECTION AND INFLUENCE IN GENDER SEGREGATED NETWORKS

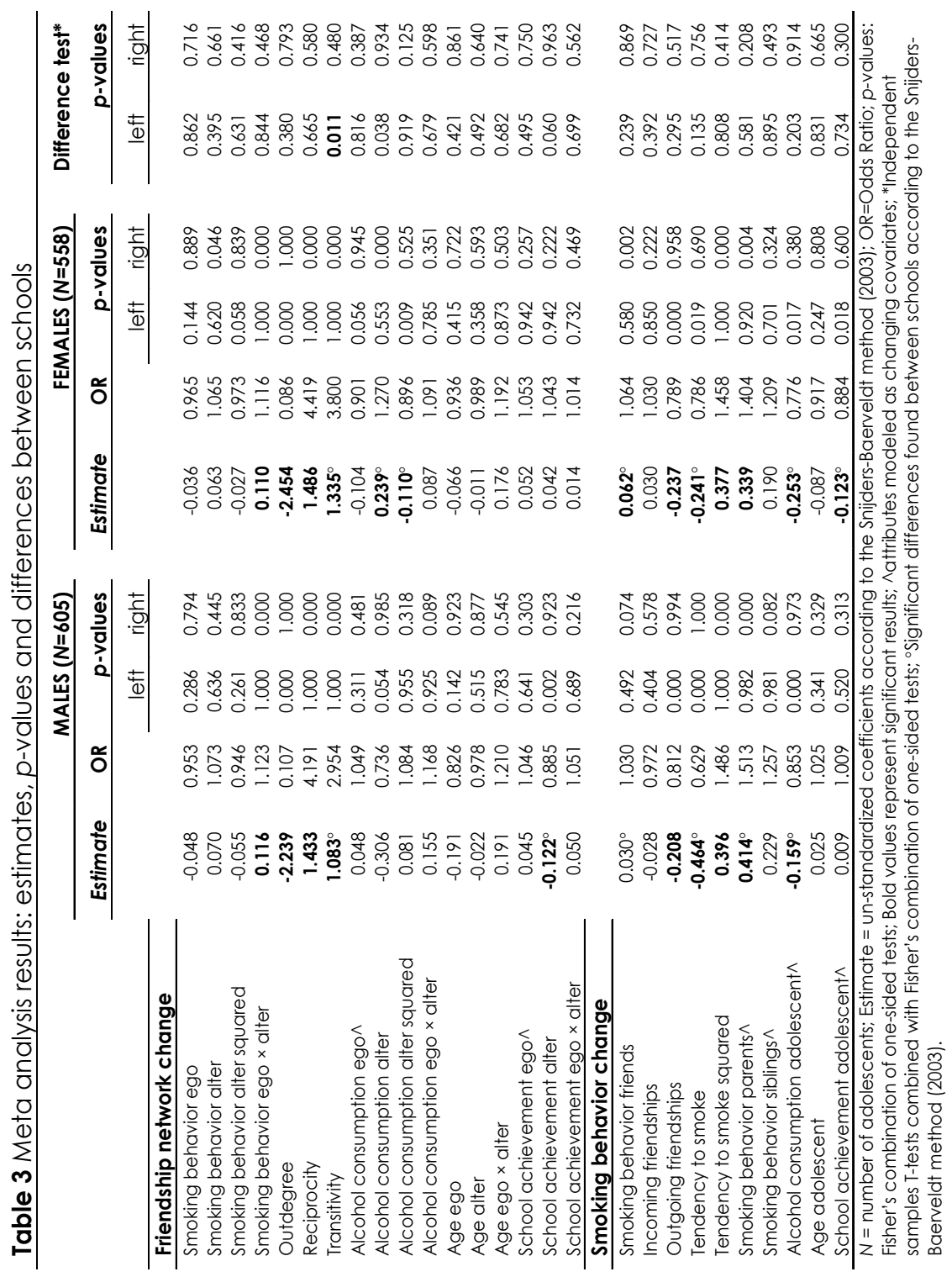




\section{The relative contribution of smoking-based selection and influence}

Figure 1 shows that the mean proportion of similarity attributed to smoking-based friendship selection was respectively $22 \%$ for males and $18 \%$ for females. The mean proportion attributed to influence of friendship networks was $15 \%$ for males and $21 \%$ for females. Inertia and trend effects accounted for $42 \%$ in male and female adolescents and other determinants of friendship and smoking only played a very small role in the explanation of smoking behavior similarity.

Figure 1 The relative contribution of smoking-based selection and influence on similarities in smoking behavior

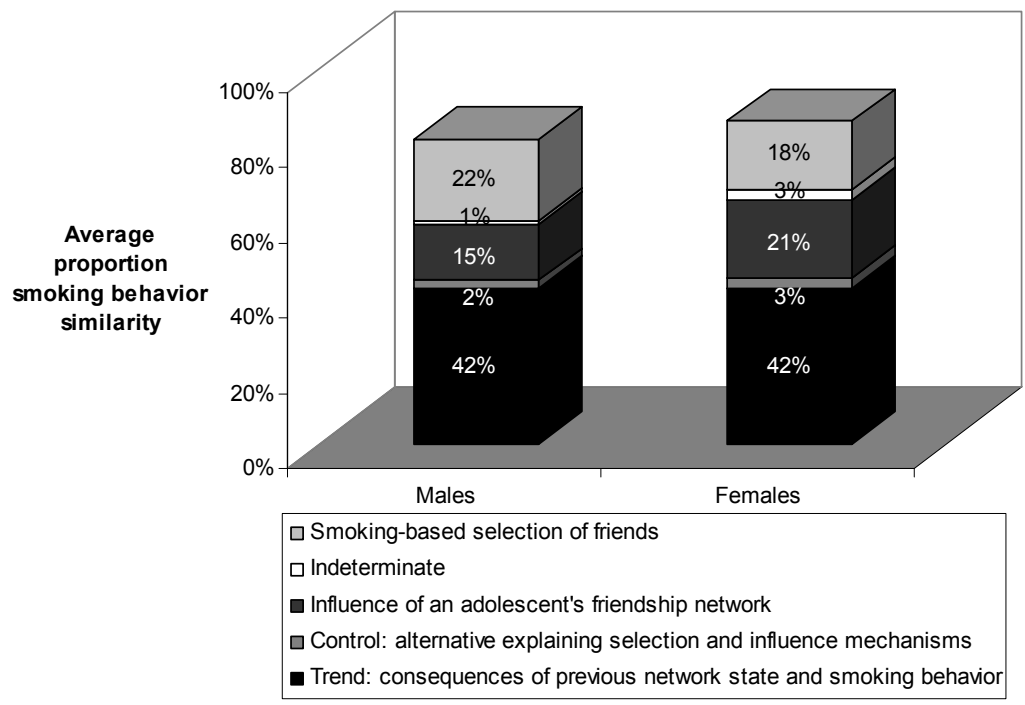




\section{DISCUSSION}

The main goal of this study was to examine differences between adolescent male and female friendship networks regarding smoking-based selection and influence processes using newly developed tools of social network analysis. Given longitudinal data, these new methods allow to draw conclusions about the empirical evidence for either of these processes, while controlling for the other process as well as for additional explanations of friendship choice and smoking dynamics that were used as general control effects.

We found evidence that males and females formed friendships based on similarities in smoking behavior. This process was similar for adolescent males and females. These results are in line with our hypothesis and earlier research reporting peer selection to be important in explaining similarities between adolescent friends with respect to smoking $[12,13,23,26,29,75]$. However, no study specifically examined the role of smoking-based selection in gender segregated friendship networks.

We found empirical support for influence of friends with respect to smoking behavior within female networks. For the male networks this effect was not significant. Although our findings seem in line with previous studies suggesting that females perceive more social pressure [130] and are more susceptible to social influences [131], the difference between males and females was not significant and parameter estimates were such that a relatively large amount of the smoking behavior similarity among male friends was explained by influence processes (Figure 1). The lack of strong support for influence processes within male networks might be explained by the fact that males more than females, foster friendship intimacy through sharing activities with friends [134], which mostly takes place outside school. Out-of-school friends with whom males share more activities or leisure time may therefore exert a more important influence compared to friends within school. Further research with a larger data base is needed to give more unequivocal results about male-female differences in this respect, and to study the dependence of these differences on the school context.

There was no evidence that reciprocation of friendship modified the effect of smoking similarity on friendship choice. In contrast with previous studies reporting stronger support for influence processes among reciprocal friends [27, 43] the strength of influence processes did not differ significantly between reciprocal and non-reciprocal friendships in the present study. However, researchers did find support 
for influence among reciprocal friends $[27,43,96]$ as well as non-reciprocal friends $[100,128]$ among different populations. More research is needed to clarify the specific role of friendship reciprocity.

Besides smoking-based selection, we controlled for a number of alternative processes explaining peer selection. Only transitivity differed significantly between males and females indicating that females showed a higher tendency to select friends that were already friends of their friends. Males and females were similar in their tendency to select arbitrary friends and reciprocate friendships. Females furthermore preferred to select other females that reported medium scores on alcohol consumption. In contrast with findings of previous studies [89] no support was found for selection based on similar alcohol consumption. However, since findings on the complete Finnish sample did show support for adolescents selecting their friends based on similar alcohol consumption [135], the lack of support may be caused by the reduced sample size due to restricting the social networks to solely males and females. In line with earlier studies arguing that boys' culture is less study oriented [136], and that school achievement is not considered to be 'cool' among boys [137, 138], males preferred to select other males scoring low on school achievement.

Regarding the alternative influence mechanisms, no significant gender differences were found. Females did smoke more when they scored low on school achievement. In line with previous studies, males and females were both influenced by parental smoking behavior $[18,75,130]$. The counter-intuitive negative effects of alcohol consumption on smoking might be explained by youngsters' choices to either smoke or start consuming alcohol.

\section{Limitations}

The following limitations of this study can be reported. First, self-reported smoking behavior was not biochemically validated. However, self-reported smoking has been found to be reliable and to correspond well with biological indicators under measurement conditions that assure anonymity [86]. The ESFA project optimized measurement conditions by guaranteeing strict confidentiality of adolescent responses [66]. Second, data were gathered from the Helsinki area only. It is conceivable that in a non-urban Finnish area peer influence might be different. Further research is needed to illuminate similarities and differences between urban and rural areas. Third, we only included friendships within school within the same school grade. Although these friends represent an important part of an adolescent's social environment, future studies should examine influence and selection in larger 
social networks that include friends of other school grades and out-of-school friends. Fourth, due to the scope of the present study we did not include various effects parents can have on their children's peer relationships. However, previous research has demonstrated that parents can have an effect on the types of friends that adolescents are selecting [139]. Future research should examine the role of parenting styles and practices in smoking-based selection processes using actor-based modeling techniques. Fifth, several included constructs were measured with one item. Future studies should include variables based on multiple questions to increase reliability and validity. Sixth, although adolescents have been known to be reliable sources to assess the smoking status of their parents [103], our results might be biased since no direct measures of parental and sibling smoking were available. Finally, we modeled selection and influence processes over three data waves without considering possible differences between the three successive data waves. However, a previous study on the same dataset indicated no differences in the main included smoking-based selection and influence effects between the three separate waves [135].

\section{Practical implications}

This study has several practical implications. First, both male and female adolescents selected friends based on similar smoking behavior, implying that prevention programs for adolescent males as well as females should focus on selection processes. Previous studies already demonstrated the importance of peer networks besides the focus on social influences $[92,116]$. Although more research is needed on the relation between attitudes and selection process, reinforcing non-smoking attitudes among males and females might be a way to increase the likelihood that they select non-smoking peers. Second, only females were significantly influenced by their friends to take up smoking and should therefore benefit from learning skills to resist social influence of friends. Although the support for influence of friends among males was weak, the effects between males and females did not differ significantly and we therefore cannot conclude that prevention programs will benefit from targeting adolescent males and females in a different way. Third, as both males and females were influenced by parental smoking behavior, the nature of this link should be investigated in more detail to learn how parents should be involved in youth smoking prevention programs. 



\section{CHAPTER 7}

General discussion 
The World Health Organization (WHO) reported smoking to be a major risk factor for the development of and death from several forms of cancer, pulmonary and respiratory diseases, cardiac diseases, and vascular diseases [140]. Despite these dangerous health consequences, current smoking rates among European adolescents are the highest in the world [119]. Adolescents continue to initiate smoking, which underlines the need for insight into different processes that might explain smoking initiation and maintenance during adolescence. Friends' smoking behavior is found to be one of the most consistent and robust predictors of adolescent smoking [14]. In a comprehensive review, Conrad and colleagues showed that friends' smoking behavior influenced adolescent smoking behavior in 15 out of 19 studies [69]. Similarities in smoking behavior among friends, however, can be caused by selection processes besides influence processes. Selection occurs when adolescents select friends based on similar smoking behavior. When smokers select smoking friends and non-smokers select non-smoking friends, smoking behavior among friends becomes similar. Social influence among friends occurs when adolescents adjust their smoking behavior in response to the example of or the direct pressure by their friends. Since in most studies peer influence and selection processes have not been disentangled, the impact of friends' smoking on adolescent smoking may be overestimated.

The aim of the present dissertation was to gain more insight in and disentangle selection and influence processes within peer friendship networks. We examined selection and influence processes in a large dataset containing behavioral and relational data of adolescents in six European countries (Denmark, Finland, the Netherlands, Portugal, Spain, and the UK) that participated as a control group in the ESFA (European Smoking Prevention Framework Approach) study [66]. Friendship networks, smoking behavior and relevant other adolescent characteristics were assessed at four waves during autumn 1998, 1999, 2000 and spring 2001. To examine the co evolution of friendship networks and smoking behavior we employed structural equation modeling and longitudinal social network modeling techniques. In this concluding chapter, the main findings of the five studies presented in this dissertation will be discussed. In addition, we will address the limitations and present some directions of future research. Finally, practical implications will be discussed. 


\section{GENERAL DISCUSSION}

\section{Similarities in smoking behavior caused by selection and influence processes}

The first question posed in the present dissertation was "Do friends select each other based on similar smoking behavior?". Findings presented in chapters two to six unequivocally show that adolescents selected each other based on similar smoking behavior. In chapter four, evidence for selection based on similarities in smoking behavior was found in each of the six European countries that participated in the ESFA study. The second question posed was "Do friends influence each other in smoking behavior?" Support for influence of friends was found in the studies reported in chapters two and three, which only included adolescents from the Netherlands. Chapter four demonstrated that support for influence of friends was not found in all of the six included European countries. Besides in the Netherlands, adolescent smoking behavior was influenced by the smoking behavior of their friends in Finland. In chapter four, an in depth examination of network autocorrelation coefficients (descriptive statistics that represent the similarity of individuals linked in a network) furthermore demonstrated that the observed similarities in smoking behavior among linked adolescents within a school network were explained more by smoking-based selection processes than by influence of friends in each of the six countries.

These findings were in line with a large body of research conducted during the past decade [30-34], arguing that the role of peer selection processes appears to be at least as significant as the role of peer influence processes during adolescence. However, in the present dissertation, several methodological pitfalls were tackled through improved research design. We avoided projection bias by using the smoking behavior as reported by the adolescents and friends themselves and considered friendship changes over time. In chapters 4-6, we were further able by conducting advanced social network analysis to model the occurrence of changes in friendships and smoking behavior between consecutive observation moments in a more realistic way, to control for alternative selection mechanisms, and to allow network dependencies to occur [59].

In sum, adolescents demonstrated a strong propensity to select friends based on similar smoking behavior. The support for influence processes among friends was weaker than expected based on results of previous research, implying that other factors might play a more important role in determining adolescent smoking behavior. 


\section{The role of friendship reciprocity in influence and selection processes}

In chapters two, three, and five the specific role of friendship reciprocity in influence processes was examined. In most previous studies, the role of friendship reciprocity was not considered when examining selection and influence processes. By taking reciprocity into account, we were able to examine possible influences of four types of peers or friends in the adolescents' social networks. Table 1 describes each of the examined peer relationships.

Table 1 Friendship formations

Wannabe friend
Fesired friend

In chapter two a structural equation model was tested which made a distinction between reciprocal friends and wannabe friends, whereas in chapter three a model was tested which discriminated between desired friends, reciprocal friends and former friends. Findings from chapters two and three showed that in the Netherlands adolescents were not influenced by wannabe friends nor former friends, but were influenced by their desired friends' smoking behavior. Results regarding the influence of reciprocal friends were mixed. The study reported in chapter three did not find significant support for influence among reciprocal friends, which contradicts the results of the earlier study reported in chapter two. In chapter three, however, a more refined model was tested which allowed a better control for covariates of adolescent smoking behavior which might have resulted in these contradicting findings. In chapter five differences between influence of desired and reciprocal friends were examined in a Finnish sample using social network analysis techniques. Support was found for Influence of desired friends as well as reciprocal friends among Finnish adolescents. 
The role of friendship reciprocity in smoking-based selection was also examined in the same three chapters. Findings indicated that reciprocity did not play an important role in smoking-based selection of friends as support for selection of reciprocal as well as desired friends was found in the Dutch studies reported in chapters two and three. However, the social network analysis reported in chapter five, using the Finnish ESFA dataset, clearly showed that friendship reciprocity did play a role in that specific sample. Adolescents in Finland had a significant stronger propensity to select desired friends based on similar smoking behavior compared to the propensity to select reciprocal friends based on similarities in smoking behavior.

Desired friends, those friends that did not yet return the friendship, seem to be selected based on similarities in smoking behavior and seem to have the power to influence their future friend's smoking behavior before a reciprocal friendship is formed. These findings suggest that desired friends and reciprocal friends are different agents within an adolescent's social network, that they can be associated with different selection and influence processes, and should be studied separately. Researchers have argued that adolescents were more strongly inclined to adapt to a desired friend's norms and behaviors because of their intrinsic needs to make the friendship mutual and adapting to the smoking behavior of a desired friend might be one way to achieve this [100, 141]. Furthermore, the actual influence of a desired friend may differ from the influence within a reciprocal friendship. Although one of the strengths of the studies presented in this dissertation is that research findings are based on analyses with actual smoking behavior measures of friends instead of adolescents' perceptions of the smoking behavior of friends, it is feasible that the modeling of smoking behavior will be based more strongly on perceptions of smoking behavior when it involves a desired friend in contrast to a reciprocal friend. Perceptions of smoking behavior may also differ and be less accurate for desired friends compared to reciprocal friends.

In sum, our findings suggest that desired and reciprocal friends can be associated with different selection and influence effects and should therefore be distinguished in future research. In addition, desired friends appeared to be selected based on similarities in smoking behavior and to influence their future friend's smoking behavior. Further research is needed to examine the specific role of desired friends in more detail. 


\section{Changes over time in influence and selection processes}

In chapter three changes in selection and influence were examined during three successive waves using structural equation modeling in a Dutch sample. Findings indicated that in the Netherlands, selection based on similar smoking behavior decreased over time. Results furthermore only showed support for influence of desired friends during the third/last wave, indicating that influence of desired friends increased from early to mid adolescence.

In chapters five and six, the relative contribution of smoking-based selection and influence processes to observed smoking behavior similarity between friends was examined among Finnish adolescents by studying changes in network autocorrelation coefficients over time. In line with the findings of chapter three, the proportion of smoking behavior similarities explained by smoking-based selection processes decreased over time. Results furthermore showed a decrease in the impact of friend influence processes in causing smoking behavior similarities among friends. However, in these social network studies, changes over time were not examined separately for desired and reciprocal friends. In this manner, we cannot exclude the possibility that influence of desired friends in particular increased, while influence of reciprocal friends decreased.

It is known that conformity increases during middle childhood, peaks in middle adolescence and then decreases [142, 143]. Young adolescents affiliate with peer groups by confirming to group norms. When adolescents grow older, they form a more autonomous sense of self, group affiliation becomes less important and conformity decreases [144]. The decrease of smoking-based selection of friends with age may be explained by children's changing selection criteria. Older children are known to choose friends based on criteria that are more relevant for later phases of friendship, friendship satisfaction and durability [46].

In sum, influence and selection processes seemed to decrease from early to mid adolescence. In addition, our findings indicated that the specific influence of desired friends may increase over time. More research is needed to confirm our findings in other samples.

\section{The role of gender in selection and influence processes}

The final goal of this dissertation was to examine the role of gender in selection and influence processes. In chapters four and five, findings demonstrated in line with previous studies that adolescents had the propensity to choose friends based on similar gender [52]. Chapter six specifically addressed the role of gender in smoking- 
based selection and influence processes by studying friendship and smoking behavior dynamics in male and female friendship networks. Slightly stronger evidence was found for influence among adolescent females compared to males which is in line with previous research demonstrating that females perceived more social pressure [130] and were more susceptible to social influences [131].

Differences in attachment styles between adolescents may explain variations found in individual susceptibility to influences of friends [145]. Attachment styles consist of two major dimensions: anxiety and avoidance. Individuals expressing an anxious attachment style, are anxious to be accepted, afraid of abandonment, or over concerned with approval. Individuals who are avoidant attached are distant and avoid closeness [146]. Adolescents demonstrating an anxious attachment to a peer group may identify more strongly with that group for fear of being rejected. This may result in engaging in risk behaviors such as cigarette smoking and alcohol consumption in order to be noticed or accepted. Throughout childhood and adolescence, females are known to differ from males in their attachment styles [147, 148]. Further research is needed to explore the relationships between attachment styles and smoking-based selection and influence processes among males and females.

\section{Structural Equation Modeling versus Social Network Analysis}

In the present dissertation selection and influence processes were disentangled initially by using structural equation modeling techniques and during the later phases by using actor-oriented models in longitudinal social network analysis. The shortcomings of conventional analysis techniques and more sophisticated and advanced analysis techniques such as structural equation modeling have been discussed extensively throughout this dissertation. Three main issues are fundamental for the separation of selection and influence processes: (1) modeling possible changes in friendships and smoking behavior between consecutive observation moments (2) controlling for alternative selection mechanisms, and (3) allowing network dependencies to occur [59]. When the set of friends at one observation moment is different from the set of friends at the next observation moment, in SEM it is necessary to make decisions, with arbitrary components, to specify whose influence plays a role in such cases. Furthermore, if the set of friends remains the same, a change might occur as well as a change back to the original value during the same period in between two discrete observations. If data would be completely observed, the problem of alternative mechanisms remains in SEM analyses. The selection of 
friends takes place not only based on attributes such as smoking behavior but also on the basis of current network structure. One example is 'transitivity', which is a structural network mechanism known to play a strong role in friendship formation. Transitivity reflects the tendency to select those friends that are already friends of your friends regardless of their smoking behavior $[60,61]$. Not controlling for such mechanisms would mistakenly lead to diagnosing selection based on similar smoking behavior. Third, when analyzing data of a complete network, the smoking behavior score of a specific individual can appear in more than one observation in the dataset. Smoking behavior of many individuals will also appear as smoking behavior scores of friends in observations of other individuals. This phenomenon violates independence of observations, a crucial assumption of SEM.

By constructing actor-based models for the co-evolution of networks and behavior as implemented in the SIENA software, we were able to overcome those shortcomings and succeeded in disentangling smoking-based selection and influence processes. Although we recommend future researchers to use these advanced social network analysis techniques to solve empirical problems related to selection and influence processes, this method also has some limitations. First, compared to SEM, conducting social network analyses can be rather timeconsuming for larger networks because of the iterative process used to estimate parameters. Second, during the course of this research social network analysis was limited to dichotomous network ties (having a friendship or having no friendship). It was not possible to model different strengths or qualities of friendship ties. Third, there are no conventional metric effect sizes in SIENA such as model fit indices or R-squares that are available in SEM. However, the resulting T-statistics are standardized and may be therefore used to assess the relative strength of the various parameter estimates but it is not yet possible to compare results with effect size statistics calculated via other statistical techniques [107].

In conclusion, when examining influence and selection processes the use of actor-oriented models for the co-evolution of networks and behaviour is recommended. Despite the current limitations of this new method, it tackles three fundamental methodological problems of conventional analysis techniques. 


\section{LIMITATIONS AND SUGGESTIONS FOR FUTURE RESEARCH}

First, the social networks of adolescents examined in the present dissertation were limited to friendships within the same school grade. Adolescents also tend to select friends outside their own school grade or outside the school environment and may well be influenced by those friends, which may explain the somewhat weaker evidence found for influence processes among friends. Although focusing on school grade networks has some advantages, such as controlling for the availability of friends within the network and to some extent controlling for selection of friends based on similarities in attributes other than included in the present research, future social network studies focusing on selection and influence processes during adolescence should aim to examine all friendships inside and outside the school environment.

Second, in this dissertation we examined smoking-based selection and influence processes within friendship dyads. In the social network studies presented in chapters four to six, we controlled for the formation of triads (a small group of three adolescents that share friendships with each other). However, we did not examine whether adolescents select friends of friends based on different criteria and whether adolescents part of a triad experience different influence processes compared to adolescents that are part of a friendship dyad. Ties in a triad are known to be qualitatively different from dyadic ties [149]. Dyads preserve more individuality compared to triads, in a dyad, individuals have more bargaining power than in a triad, and conflicts are more readily managed and resolved in a triad [150]. Furthermore, Social Identity theory states that smoking homogeneity of behavior develops within groups as group members modify their behavior to fit the norms that are central to the social identity of the group [151]. This process may be more pronounced during adolescence as identity is yet to be formed. By selecting friends, adolescents thus choose their own peer group and context, which will determine the kind of influences they will experience. Influence can play different roles among dyads, triads, or larger social groups. Influence of a single friend can be concordant with the influence of the social crowd, but can also differ. Future research should examine smoking-based selection and influence processes in the multiple dimensions of adolescent's social networks.

Third, among adolescents in the network, either a friendship tie was directed from one adolescent to another or no friendship existed. The SIENA software used during the course of this dissertation did not allow us to include gradations of tie 
values such as friendship quality or friendship strength. Furthermore we did not consider other relationships between adolescents such as negative ties or romantic ties. Negative ties might exist among adolescents who are or have been in a fight and whose friendship ended. During adolescence, romantic relationships can also evolve which can be important for peer status and facilitate relationships with other adolescents. The effects of a break-up can undermine an adolescent's standing and might even result in losing one's place in the social network [152]. Future social network research needs to focus on positive as well as negative relationships and should model different types of relationships between adolescents with graded tie values as reported by the adolescents themselves.

Fourth, in the present dissertation peer influence was assumed to exist based on the association between the actual smoking behavior of two adolescents in a social network. Whether peers offer each other cigarettes, model smoking behavior, exert pressure to smoke, or adapt to smoking norms of social crowds was not examined. Whether peers also exert influence on smoking-related attitudes and beliefs besides influence on actual smoking behavior was also beyond the scope of the present dissertation. A broader spectrum of influence processes should be studied in the future by using actor-based models for the co-evolution of networks and behavior.

Finally, although we controlled for differences between schools, we did not investigate possible effects of school factors such as school size and smoking bans in schools on smoking-based selection and influence effects. Furthermore, there is a growing body of research indicating that adolescents from various ethnic groups differ in their selection of similar friends $[153,154]$. Adolescents might use different criteria when selecting friends, rooted in unique historical and cultural circumstances. Similarities in smoking behavior for example may be a strong selection criterion in many but not all cultures. Future research should consider a multilevel approach and investigate the role of school characteristics and cultural differences in smokingbased selection and influence processes among adolescents. 


\section{PRACTICAL IMPLICATIONS}

Findings from the present dissertation suggest that current prevention programs such as mass-media campaigns and school smoking prevention interventions should focus on adolescents' dynamic social environment besides focusing on the adolescent as an individual. Strong support was found indicating that adolescents selected friends based on similar smoking behavior. By selecting friends, they also select their own future social environment in which they will experience future influences. It is important to increase adolescents' knowledge about influences of their social environment and more specifically their own role in that when selecting smoking friends. By strengthening attitudes and norms less favorable to smoking among adolescents, interventions may be able to decrease the tendency to select smokers as friends and increase the popularity of non-smokers.

Support for influence was also found. Although the support from our studies was only weak, it is very well feasible that the role of influence among adolescents is larger when larger social networks are studied containing friends within as well as outside school. An informal peer-led intervention program that adopts the social influence approach in combination with a social network approach can be successful in reducing adolescent smoking behavior [116]. Popular non-smoking adolescents that are for example regarded as opinion leaders by their peers can be targeted to become key individuals that may influence adolescents in real time while they make decision whether or not to smoke.

Specifically those adolescents that have many desired friends can be regarded as a high risk group since adolescents were found to be influenced by their desired friends to adapt their smoking behavior. By using a social network approach, intervention programs could furthermore identify those adolescents with many desired friends that smoke. Changing the image of smokers, changing the adolescents' attitudes, teaching skills to resist peer pressure and increasing selfefficacy to resist smoking may all help to reduce their susceptibility to these influences.

Our findings demonstrated that smoking-based selection and influence processes may differ for adolescents in different social positions. Previous research furthermore indicated that adolescents of different social status might smoke for different reasons [155]. These findings imply that intervention programs aimed at preventing smoking behavior among adolescents would benefit from considering the wider peer group structure. Computer-based interventions can be developed that 
incorporate a component to assess the social network structure of an adolescent and enable tailoring messages on the specific needs of adolescents in different social positions.

Since our findings only weakly indicated different influence processes for girls and boys, we can not firmly confirm that gender specific strategies are needed to counter smoking uptake during early adolescence. However, our finding were in line with previous studies suggesting that girls perceive more social pressure [130] and are more susceptible to social influences [131]. More research is needed to clarify the role of gender in smoking-based selection and influence processes.

Although it was not the main focus of the research presented in the present dissertation, we did find support for influence of parental smoking behaviour. During early adolescence, youngsters were influenced by smoking behavior of their parents. During mid adolescence, this influence diminished and peer smoking behavior increasingly affected adolescent smoking behavior. Besides focusing on actual smoking behavior of parents, a substantial body of research focused on parenting styles and anti-smoking parenting practices. Some studies suggested that children of authoritative parents who have high levels of control (monitoring and consistent discipline) and acceptance (nurturance, warmth and attachment) are less likely to initiate smoking $[156,157]$. Other findings, however, could not confirm a significant association between parental control and smoking onset [158]. Having rules and discussions about smoking generally are found to be helpful in prevention smoking by youngsters [159-161]. Previous research furthermore demonstrated that parents can have an effect on the friendship relationships of their child by guiding and monitoring their child, and prohibiting contact with some youngsters [139]. However, it is important to replicate these findings using social network analysis techniques which can take dependencies caused by the friendship network structure of adolescents into account, and can represent changes in adolescents' friendship networks and smoking behavior in a realistic way. Programs targeting youngsters in early adolescence should include parents in prevention programmes but more research still is needed to examine the specific role parents can play in their children's smoking behavior.

The findings of the present dissertation also don't deny there still is a lot to be gained by national anti-smoking policies. National policies can help prevent imitation of smoking behaviour. Prohibiting smoking in social settings and public places such as schools, cinema's, restaurants, bars, and public transport will not only protect nonsmokers from passive smoking, but also presumably effectively decrease smoking 
uptake in adolescence. A longitudinal study in Massachusetts for example found that besides a strong increase of quit attempts among adults, young people living in towns with strong compared to weak smoking regulations in restaurants had less than half the odds of becoming an established smoker [162, 163]. Comprehensive bans on tobacco marketing, forcing plain packaging and reducing positive media images of smoking may also help to create non-smoking norms among youngsters, and reduce the risk that adolescents select a social environment that will influence them to start smoking. In the UK, the evaluation of a comprehensive ban on tobacco advertising and promotion showed a reduction of young people's awareness of tobacco marketing, as well as their susceptibility to smoking [164]. A comprehensive review of the entertainment media's role in promoting tobacco use found that anti-tobacco advertisements before movies can partially counteract the impact of tobacco portrayals in movies among American and Australian adolescents [165]. Future research should examine whether strong national anti-smoking policies can indeed also specifically reduce peer influence to start smoking and increase healthy friendship choices among youngsters by establishing a non-smoking norm and specifying what is socially desirable. 

SUMMARY 
Tobacco use is the fourth most common risk factor for disease worldwide and the second major global cause of mortality [1]. In most Western countries, the smoking prevalence increases during adolescence [2, 3]. Among 13-year olds, the prevalence of regular smoking varies from $3.5 \%$ to $12.5 \%$ and increases to $17 \%$ to $24.5 \%$ for 15 -year olds [4]. Once adolescents start to smoke, they are more likely to become a regular smoker due to the physiological dependence on nicotine [5-7]. Insights into the different processes that might explain smoking initiation and maintenance during adolescence may benefit the development of prevention programs and adaptation of policies regarding effective smoking prevention.

A substantial body of research suggests that smoking behavior is often similar among friends $[9,10]$. This similarity may occur as a result of two different processes: friendship selection and influence. Selection occurs when adolescents select friends with similar smoking behavior during the formation of a new friendship [12, 13]. Social influence among friends occurs when adolescents adjust their smoking behavior in response to the example of or the direct pressure by their friends [12-14]. During the past few decades, several studies have tried to separate selection from influence processes in adolescent deviant behaviors such as smoking behavior, marijuana use, alcohol consumption and delinquency [12, 13, 23-34]. The main conclusion of this substantial body of research is that the role of selection processes appears to be at least as significant as the role of peer influence, certainly during adolescence. However, after decades of research attempting to disentangle selection and influence processes, some important limitations remain present.

The aim of the present dissertation was to gain more insight in and disentangle selection and influence processes within peer friendship networks by overcoming several of the main limitations of previous research which are discussed in detail in chapter 1. We examined selection and influence processes in a large dataset containing behavioral and relational information on adolescents in six European countries that participated as a control group in the ESFA (European Smoking Prevention Framework Approach) study [66]. Friendship networks, smoking behavior and relevant other adolescent characteristics were assessed at four waves during autumn 1998, 1999, 2000 and spring 2001. To examine the co-evolution of friendship networks and smoking behavior we employed structural equation modeling (chapters 2 and 3) and longitudinal social network modeling techniques (chapters 4, 5, and 6). Of the various statistical techniques used by previous researchers to disentangle selection and influence effects, until recently structural equation modeling (SEM) remained the best option as it had the least shortcomings. 
This statistical technique is able to examine selection and influence processes simultaneously, while mutually controlling for each process. However, this method still is limited by not being able to address three main issues that are fundamental for the separation of selection and influence processes: (1) modeling the possible occurrence of changes in friendships and smoking behavior in between consecutive observation moments (2) controlling for alternative selection mechanisms, and (3) allowing network dependencies to occur [59]. However, the advanced social network analysis method using actor-based models for co-evolving friendship networks and smoking behavior as implemented in the SIENA (Simulation Investigation for Empirical Network Analyses) software is capable of tackling these three limitations.

The first question posed in the present dissertation was "Do friends select each other based on similar smoking behavior?". Findings presented in chapters two to six unequivocally showed that adolescents selected each other based on similar smoking behavior. The second question posed was "Do friends influence each other in smoking behavior?" Support for influence of friends was found in the studies reported in chapters two and three, which only included adolescents from the Netherlands. Chapter four demonstrated that support for influence of friends was not found in all of the six included European countries. Besides in the Netherlands, adolescent smoking behavior was influenced by the smoking behavior of their friends in Finland.

Since the role of friendship reciprocity was not considered when examining selection and influence processes in most previous studies, the specific role of friendship reciprocity in influence and selection processes was studied in chapters two, three and five. Our findings suggested that desired and reciprocal friends were associated with different selection and influence effects and should therefore be distinguished in future research. In addition, desired friends appeared to be selected based on similarities in smoking behavior and to influence their future friend's smoking behavior. Further research is needed to examine the specific role of desired friends in more detail.

The fourth question posed in the present dissertation was "Do influence and selection processes change over time?". In chapter three changes in selection and influence were examined during three successive waves using structural equation modeling in a Dutch sample. Findings indicated that selection based on similar smoking behavior decreased over time. Results furthermore only showed support for influence of desired friends during the third/last wave, indicating that influence of 
desired friends increased from early to mid adolescence. In line with the findings of chapter three, chapter five and six showed that the proportion of smoking behavior similarities explained by smoking-based selection processes decreased over time. Results furthermore showed a decrease in the impact of friend influence processes in causing smoking behavior similarities among friends. However, in these social network studies, changes over time were not examined separately for desired and reciprocal friends.

The final goal of this dissertation was to examine the role of gender in selection and influence processes. In chapters four and five, findings demonstrated in line with previous studies that adolescents had the propensity to choose friends based on similar gender [52]. Chapter six specifically addressed the role of gender in smoking-based selection and influence processes by studying friendship and smoking behavior dynamics in male and female friendship networks. Slightly stronger evidence was found for influence among adolescent females compared to males.

In the concluding chapter (chapter 7), the main findings of our studies were summarized and discussed. The research presented in the present dissertation has led to some new insights regarding the co-evolution of friendship networks and influence processes during adolescence. In addition, we discuss the most important limitations and present recommendations for future research and practical implications. 
SAMENVATTING 
Tobaksgebruik is de vierde hoogste risicofactor voor ziekte en de tweede hoogste oorzaak van sterfte wereldwijd [1]. In het merendeel van de westerse landen ziet men een toename van roken tijdens de adolescentie $[2,3]$. $3.5 \%$ tot $12.5 \%$ van de 13 jarigen rookt regelmatig en de prevalentie van rokers neemt met de leeftijd toe gaande van $17 \%$ tot $24.5 \%$ rokers onder 15 jarigen [4]. Eens adolescenten beginnen met roken is het risico groter om een regelmatige roker te worden door het verslavingseffect van nicotine [5-7]. Inzichten in de verschillende processen die beginnend en volhardend rookgedrag kunnen verklaren tijdens de adolescentie kunnen de ontwikkeling van preventie programma's en de aanpassingen van regelgeving aangaande effectieve rookpreventie verbeteren.

Een aanzienbaar aantal wetenschappelijke studies suggereert dat vrienden vaak hetzelfde rookgedrag vertonen $[9,10]$. Deze gelijkheid kan veroorzaakt worden door twee verschillende processen: vriendschap selectie en beïnvloeding. Selectie vindt plaats wanneer adolescenten vrienden selecteren gebaseerd op gelijk rookgedrag tijdens het vormen van een nieuwe vriendschap [12, 13]. Sociale invloed onder vrienden komt voor wanneer adolescenten hun rookgedrag aanpassen gebaseerd op voorbeelden van vrienden of onder druk van vrienden [12-14]. Gedurende de afgelopen decennia hebben meerdere studies geprobeerd selectie processen van invloed processen te scheiden in de context van adolescent rookgedrag, marijuana gebruik, alcohol consumptie en crimineel gedrag [12, 13, 2334]. De hoofdconclusie van deze studies is dat de rol van selectie processen tenminste zo belangrijk is vergeleken met de rol van invloed processen, zeker gedurende adolescentie. Maar, na decennia onderzoek dat poogt selectie en invloed processen vit elkaar te rafelen, blijven nog steeds een aantal belangrijke tekortkomingen bestaan in onderzoek.

Het doel van dit proefschrift was meer inzicht te verkrijgen in en het ontrafelen van selectie en invloed processen binnen vriendschapsnetwerken en daarbij een aantal belangrijke beperkingen overwinnen van vroegere studies dewelke gedetailleerd zijn beschreven in hoofdstuk 1. We hebben selectie en invloed processen bestudeerd in een grote dataset die informatie bevat over gedragingen en relaties van adolescenten in zes europese landen die als controle groep hadden deelgenomen in de ESFA (European Smoking Prevention Framework Approach) studie [66]. Vriendschapsnetwerken, rookgedrag en relevante andere eigenschappen van adolescenten waren verzameld op vier tijdstippen gedurende herfst 1998, 1999, 2000 en lente 2001. Om de co-evolutie van vriendschapsnetwerken en rookgedrag te onderzoeken hebben we structural equation modeling (SEM) 
analyse technieken gebruikt (hoofdstuk 2 and 3) en longitudinale sociale netwerk analyse technieken (hoofdstuk 4, 5, and 6). Van de vele statistische technieken gebruikt door vroegere onderzoekers om selectie en invloed effecten van elkaar te scheiden, bleef SEM tot op heden de beste optie gezien het de minste tekortkomingen had. Deze statistische techniek kan selectie en invloed processen tegelijk onderzoeken, terwijl het ene proces controleert voor het andere. Deze methode is echter gelimiteerd gezien drie fundamentele opties, cruciaal voor het scheiden van selectie in invloed processen niet mogelijk zijn: (1) het modelleren van mogelijke veranderingen in vriendschappen en rookgedrag tussen twee opeenvolgende observatie momenten (2) het controleren voor alternatieve selectie mechanismen, en (3) het toelaten van afhankelijkheden veroorzaakt door het netwerk [59]. De geavanceerde sociale netwerk analyse methode die gebruik maakt van actor-based models voor de co-evolutie van vriendschap netwerken en rookgedrag geïmplementeerd in de SIENA (Simulation Investigation for Empirical Network Analyses) software houdt wel rekening met deze drie fundamentele tekortkomingen.

De eerste onderzoeksvraag bestudeerd in dit proefschrift luidt: "Selecteren vrienden elkaar gebaseerd op gelijk rookgedrag?". De bevindingen gepresenteerd in hoofdstukken twee tot zes tonen eensgezind aan dat adolescenten elkaar selecteerden gebaseerd op gelijk rookgedrag. De tweede onderzoeksvraag luidde: "Beïnvloeden vrienden elkaars rookgedrag?". Bewijs voor invloed van vrienden is gevonden in de studies gerapporteerd in hoofdstukken twee en drie, in welke enkel Nederlandse adolescenten waren geïncludeerd. Hoofdstuk vier toont dat bewijs voor invloed van vrienden niet in alle zes Europese landen van de ESFA studie was gevonden. Adolescent rookgedrag was enkel beïnvloed door rookgedrag van vrienden in Nederland en Finland.

Gezien de rol van vriendschap reciprociteit niet is meegenomen in de meeste studies die selectie en invloed processen onderzochten, was de specifieke rol van reciprociteit bestudeerd in hoofdstukken 2, 3 en 5. Onze bevindingen suggereren dat gewenste vrienden (niet reciproque of unilaterale vrienden) en reciproque vrienden geassocieerd zijn met verschillende selectie en invloed processen en dus apart onderzocht moeten worden in toekomstig onderzoek. Daarenboven bleek dat adolescenten hun gewenste vrienden selecteerden gebaseerd op gelijk rookgedrag en dat ze door deze gewenste vrienden ook beïnvloed werden. Meer onderzoek is nodig om de rol van gewenste vrienden in detail te onderzoeken. 
De vierde onderzoeksvraag in dit proefschrift luidde: "Veranderen invloed en selectie processen over de tijd?". In hoofdstuk drie hebben we in de Nederlandse dataset de veranderingen in selectie en invloed processen onderzocht in drie opeenvolgende waves gebruik makende van structural equation modeling technieken. Resultaten toonden aan dat selectie gebaseerd op gelijk rookgedrag afnam over de tijd heen. Verder toonden de resultaten enkel ondersteuning voor invloed van gewenste vrienden tijdens de derde/laatste wave. Dit impliceert dat invloed van gewenste vrienden toeneemt van vroege naar mid adolescentie. In overeenstemming met de bevindingen uit hoofdstuk drie, tonen de resultaten van hoofdstuk vijf en zes dat de proportie gelijk rookgedrag dat verklaard wordt door selectieprocessen gebaseerd op rookgedrag afnam met de tijd. Daarenboven toonden de resultaten ook een afname van de impact van invloed van vrienden. In deze sociale netwerkstudies werden veranderingen over de tijd heen echter niet afzonderlijk onderzocht voor gewenste en reciproque vrienden.

Als laatste is de rol van geslacht onderzocht in selectie en invloed processen. De bevindingen van hoofdstukken 4 en 5 toonden in overeenstemming met vorige studies aan dat adolescenten de neiging hadden vrienden te selecteren van hetzelfde geslacht [52]. In hoofdstuk 6 onderzochten we de rol van geslacht in selectie en invloed processen door de dynamieken van vriendschap en rookgedrag apart te onderzoeken in meisjes vriendschapsnetwerken en jongens vriendschaps netwerken. Bewijs voor invloed was iets sterker binnen de meisjes vriendschapsnetwerken in vergelijking met de jongens vriendschaps netwerken.

In het allerlaatste hoofdstuk (hoofdstuk 7), zijn de belangrijkste resultaten van onze studies samengevat en besproken. Het onderzoek van dit proefschrift heeft tot nieuwe inzichten geleid aangaande de co- evolutie van vriendschapsnetwerken en rookgedrag tijdens adolescentie. Daarnaast worden de belangrijkste tekortkomingen besproken, aanbevelingen gedaan voor toekomstig wetenschappelijk onderzoek en de implicaties voor preventie voorgesteld. 
REFERENCES 
1. WhO (WORLD Health ORganization) (2007) http://www.who.int/tobacco/ health_priority/en/.

2. US Department of Health and Human Services (1994) Preventing tobacco use among young people: a report of the Surgeon General (Atlanta, Georgia US Government Printing Office Publication No S/N 017-001-00491-0).

3. ChASSIN, L., PRESSON, C. C., ROSE, J. S. \& SHERMAN, S. J. (1996) The natural history of cigarette smoking from adolescence to adulthood: Demographic predictors of continuity and change, Health Psychology, 15, 478-484.

4. King, A., World, B., TudOR-SMith, C. \& Harel, Y. (1996) The health of youth. A cross-national survey. WHO-regional-publications., European-series, 69, 1-222.

5. FergusSON, D. M., LYNSKEY, M.T., \& HORWOOD, L. J. (1995) The role of peer affiliations, social, family and individual factors in continuities in cigarette smoking between childhood and adolescence, Addiction, 90, 647-659.

6. Prokhorov, A. V., Pallonen, U. E., Fava, J. L., Ding, L. \& Niaura, R. (1996) Measuring nicotine depence among high-risk adolescent smokers, Addictive Behaviors, 21, 117-127.

7. STANTON, W. R. (1995) DSM-III-R tobacco dependence and quitting during the adolescence, Addictive Behaviors, 35, 190-196.

8. Bauman, K. E., Fisher, L. A., Bryan, E. S. \& Chenoweth, R. L. (1984) Antecedents, subjective expected utility, and behavior: A panel study of adolescent cigarette smoking, Addictive Behaviors, 9, 121-36.

9. Elser, J. R., MORGan, M., GAMmAGe, P., BroOKs, N. \& KirBY, R. (1991) Adolescent health behaviour and similarity-attraction: Friends share smoking habits (really), but much else besides, British Journal of Social Psychology, 30, 339348.

10. ENNET, S. T., BAUMAN, K. E. \& KOCH, G. G. (1994) Variability in cigarette smoking within and between adolescent friendship cliques, Addictive Behaviors, 19, 295-305.

11. Sussman, S., Dent, C. W., Stacy, A. W. et al. (1990) Peer-group association and adolescent tobacco use, Journal of Abnormal Psychology, 99, 349-352.

12. Fisher, L. A. \& BAUMAN, K. E. (1988) Influence and selection in the friendadolescent relationship: Findings from studies of adolescent smoking and drinking, Journal of Applied Social Psychology, 18, 289-314.

13. EnNet, S. T. \& Bauman, K. E. (1994) The Contribution of Influence and Selection to Adolescent Peer Group Homogeneity: The Case of Adolescent Cigarette Smoking, Journal of Personality and Social Psychology, 67, 653-663. 
14. FLAY, B. R., HU, F. B., SIDDIQUI, O. et al. (1994) Differential influence of parental smoking and friends' smoking on adolescent initiation and escalation of smoking, Journal of Health and Social Behavior, 35, 248-265.

15. BANDURA, A. (1986) Social foundations of thought and action: A social cognitive theory (New York, Prentice-Hall).

16. BANDURA, A. (1977) Social learning theory (Englewood Cliffs, Prentice-Hall).

17. De Vries, H., Backiler, E., KoK, G. \& DiJkstra, M. (1995) The impact of social influences in the context of attitude, self-efficacy, intention, and previous behavior as predictors of smoking onset, Journal of Applied Social Psychology, 25, 237-257.

18. AVenevoli, S. \& Merikangas, K. R. (2003) Familial influences on adolescent smoking, Addiction, 98, 1-20.

19. Chuang, Y. C., Ennet, S. T., Bauman, K. E. \& Foshee, V. A. (2005) Neighborhood influences on adolescent cigarette and alcohol use: Mediating effects through parent and peer behaviors, Journal of Health and Social Behavior, 46, 187-204.

20. MOORE, L., ROBERTS, C. \& TUdOR-SMith, C. (2001) School smoking policies and smoking prevalence among adolescents: multilevel analyses of crosssectional data from Wales, Tobacco Control, 10, 117-123.

21. UNGER, J. B. \& CHEN, X. (1999) The role of social networks and media receptivity in predictnig age of smoking initiation. A proportional hazard model of rist and protective factors, Addictive Behaviors, 24, 371-381.

22. Distefan, J. M., Gilpin, E. A., Sargent, J. D. \& Pierce, J. P. (1999) Do movie stars encourage adolescents to start smoking? Evidence from California, Preventive Medicine, 28, 1-11.

23. COHEN, J. M. (1977) Sources of peer group homogeneity, Sociology of Education, 50, 227-241.

24. KANDEL, D. B. (1978) Homophily, selection and socialization in adolescent friendship, American Journal of Sociology, 84, 427-436.

25. KIRKE, D. M. (2004) Chain reactions in adolescents' cigarette, alcohol and drug use: similarity through peer influence or the patterning of ties in peer networks?, Social Networks, 26, 3-28.

26. Engels, R. C. M. E., KNibBe, R. A., Drop, M. J. \& DE HAAN, Y. T. (1997) Homogeneity of cigarette smoking within peer groups: influence or selection?, Health Education \& Behavior, 24, 801-811. 
27. Urberg, K. A., Luo, Q., Pilgrim, C. \& Degirmencioglu, S. M. (2003) A two-stage model of peer influence in adolescent substance use: individual and relationship-specific differences in susceptibility to influence, Addictive Behaviors, 28, 1243-1256.

28. SimOnS-MORTON, B. G. \& CHEN, R. S. (2006) Over time relationships between early adolescent and peer substance use, Addictive Behaviors, 31, 1211-1223.

29. De Vries, H., Candel, M., Engels, R. \& MerCKen, L. (2006) Challenges to the peer Influence Paradigm: Results for 12-13 year olds from six European countries from the European Smoking Prevention Framework Approach study, Tobacco Control, 15, 83-89.

30. Hall, J. A. \& VAlENTE, T. W. (2007) Adolescent smoking networks: the effects of influence and selection on future smoking, Addictive Behaviors, 32, 3054-3059.

31. Hoffman, B. R., Monge, P. R., Chou, C.-P. \& Valente, T. W. (2007) Perceived peer influence and peer selection on adolescent smoking, Addictive Behaviors, 32 , 1546-1554.

32. Wills, T. A. \& CLEARY, S. D. (1999) Peer and adolescent substance use among 6th-9th graders: latent growth analyses of influence versus selection mechanisms, Health Psychology, 18, 453-463.

33. KRAUTH, B. (2004) Peer effects and selection effects on smoking among Canadian youth, Canadian Journal of Economics, 38, 735-757.

34. WANG, M. Q., EDDY, J. M. \& FITZHUGH, E. C. (2000) Smoking acquisition: peer influence and self-selection, Psychological Reports, 86, 1241-1246.

35. BAUMAN, K. E. \& ENNETT, S. T. (1996) On the importance of peer influence for adolescent drug use: commonly neglected considerations, Addiction, 91, 185-198.

36. KOBUS, K. (2003) Peers and adolescent smoking, Addiction, 98, 37-55.

37. BAUMAN, K. E. \& FISHER, L. A. (1986) On the measurement of friend behavior in research on friend influence and selection: findings from longitudinal studies of adolescent smoking and drinking, Journal of Youth and Adolescence, 15, 345-353.

38. NASH, R. (1973) Clique formation among primary and secondary school children, British Journal of Sociology, 24, 303-313.

39. CAIRNS, R. \& CAIRNS, B. (1994) Adolescents in our time: Risks and lifelines (London, Harvester Wheatsheaf). 
40. Pearson, M. \& MichelL, L. (2000) Smoke rings: Social network analysis of friendship groups, smoking and drug-taking, Drugs: Education, Prevention and Policy, 7, 21-37.

41. Turner, K. M., West, P., Gordon, J., YOUnG, R. \& SWeeting, H. (2006) Could the peer group explain school differences in pupil smoking rates? An exploratory study, Social Science \& Medicine, 62, 2513-2525.

42. URBERG, K. A. (1990) Locus of peer influence: social crowd and best friend, Journal of Youth and Adolescence, 21, 439-450.

43. PARKer, J. G. \& Asher, S. R. (1993) Friendship and friendship quality in middle childhood: links with peer group acceptance and feelings of loneliness and social dissatisfaction, Developmental Psychology, 29, 611-621.

44. Berndt, T. J. \& HoYle, S. G. (1985) Stability and change in childhood and adolescent friendships, Developmental Psychology, 21, 1007-15.

45. LADD, G. W. (1988) friendship patterns and peer status during early and middle childhood, Journal of Developmental and Behavioral Pediatrics, 9, 229-238.

46. AbOUd, F. E. \& MENDELSON, M. J. (1996) Determinants of friendship selection and quality: Developmental perspectives, in: Bukowsky, W. M., Newcomb, A. F. \& Hartup, W. W. (Eds.) The company they keep. Friendship in childhood and adolescence, pp. 87-112 (Cambridge, Cambridge University Press).

47. Snyder, M., GANGeStad, S. \& Simpson, J. A. (1983) Choosing friends as activity partners: The role of self-monitoring, Journal of Personality and Social Psychology, 45, 1061-1072.

48. Lydon, J. E., Jamieson, D. W. \& Holmes, J. G. (1993) The meaning of social exchange in the transition from acquaintanceship to friendship, Journal of Personality and Social Psychology, 73, 536-548.

49. ENRIGHT, R. D. \& LAPSLEY, D. K. (1981) Judging others who hold opposite beliefs: The development of belief-discrepancy reasoning, Child Development, 52, 1053-1063.

50. LADD, G. W. \& EMERSON, E. S. (1984) Shared knowlege in children's friendships, Developmental Psychology, 20, 932-940.

51. Camarena, P. M., Sarigiani, F. A. \& Peterson, A. C. (1990) Gender-specific pathways to intimacy in early adolescence, Journal of Youth and Adolescence, 19, 19-32.

52. MCPHERSON, M., SMITH-LOVIN, L. \& COOK, J. M. (2001) Birds of a Feather: Homophily in Social Networks, Annual Review of Sociology, 27, 415-444. 
53. Mercken, L., Snijders, T. A. B., Steglich, C. \& De VRies, H. (2008) Dynamics of adolescent friendship networks and smoking behavior: Social network analyses in six European countries, (manuscript submitted for publication).

54. BUHRMESTER, D. \& FURMAN, W. (1987) The development of companionship and intimacy, Child Development, 58, 1101-1113.

55. Lempers, L. D. \& Clark-Lempers, D. S. (1992) Young, middle, and late adolescents' comparison of the functional importance of five important relationships, Journal of Youth and Adolescence, 2, 53-96.

56. DuRELL JOHNSON, H. (2004) Gender, grade, and relationship differences in emotional closeness within adolescent friendships, Adolescence, 39, 243-255.

57. BURKE, R. J. \& WeIR, T. (1978) Sex differences in adolescent life stress, social support, and well-being, Journal of Psychology, 98, 277-288.

58. Collins, W. A. \& Laursen, B. (1992) Conflict and Relationships During Adolescence, in: Shantz, C. U. \& Hartup, W. W. (Eds.) Conflict in child and adolescent development (New York, Camebridge University Press).

59. Steglich, C., Snijders, T. A. B. \& Pearson, M. (2007) Dynamic networks and Behavior: Separating Selection from Influence, (submitted for publication).

60. Hallinan, M. T. (1974) A structural model of sentiment relations, American Journal of Sociology, 80, 364-378.

61. Holland, P. W. \& LeinHARDT, S. (1971) Transitivity in structural models of small groups, Small Group Research, 2, 107-124.

62. Snijders, T. A. B., Steglich, C. \& SChweinberger, M. (2007) Modeling the coevolution of networks and behavior, in: Van Montfort, K., Oud, H. \& Satorra, A. (Eds.) Longitudinal models in the behavioral and related sciences, pp. 41-71 (Mahwah, NJ, Lawrence Erlbaum).

63. WASSERMAN, S. (1979) A stochastic model for directed graphs with transition rates determined by reciprocity, in: Scheussler, K. F. (Ed.) Sociological Methodology, pp. 392-412 (San Francisco, Jossey-Bass).

64. SNIJDERS, T. A. B. (2001) The statistical evaluation of social network dynamics, Sociological Methodology, 31, 361-395.

65. SNIJDERS, T. A. B. (2005) Models for longitudinal network data, in: Carrington, P., Scott, J. \& Wasserman, S. (Eds.) Models and Methods in Social Network Analysis, pp. 215-247 (New York, Cambridge University Press).

66. De VRIES, H., Mudde, A., Leiss, I. et al. (2003) The European Smoking Prevention Framework Approach (ESFA): An example of integral prevention., Health Education Research, 18, 611-626. 
67. Wahlgren, D. R., HOVelL, M. F., SLYMEN, D. J. et al. (1997) Predictors of tobacco use initiation in adolescents: a two-year prospective study and theoretical discussion, Tobacco Control, 6, 95-103.

68. Chassin, L., Presson, C. C., Sherman, S. J., Corty, E. \& Olshavsky, R. W. (1984) Predicting the onset of cigarette smoking in adolescents: A longitudinal study, Journal of Applied Social Psychology, 14, 224-243.

69. CONRAD, K. M., FLAY, B. R. \& HILL, D. (1992) Why children start smoking cigarettes: predictors of onset, British Journal of Addiction, 87, 1711-1724.

70. Bailey, S. L., ENnett, S. T. \& Ringwalt, C. L. (1993) Potential mediators, moderators, or independent effects in the relationship between parents' former and current cigarette use and their children's cigarette use, Addictive Behaviors, $18,601-21$.

71. Bauman, K. E., Foshee, V. A., Linzer, M. A. \& Koch, G. G. (1990) Effect of parental smoking classification on the association between parental and adolescent smoking, Addictive Behaviors, 15, 413-422.

72. HENRIKSEN, L. \& JACKSON, C. (1998) Anti-smoking socialization: relationship to parent and child smoking status, Health Communication, 10, 87-101.

73. FLAY, B. R. (1985) Adolescent smoking: onset and prevention, Annals of Behavioral Medicine, 7, 9-13.

74. Evans, R. I., Dratt, L. M., Raines, B. E. \& ROSEnBerG, S. S. (1988) Social influences on smoking initiation: importance of distinguishing descriptive versus mediating process variables, Journal of Applied Social Psychology, 18, 925-943.

75. WEST, P., SWEETING, H. \& ECOB, R. (1999) Family and friends' influences on the uptake of regular smoking from mid-adolescence to early adulthood, Addiction, 94, 1397-1412.

76. De VRIES, H., DiJK, F., Wetzels, J. et al. (2006) The European Smoking prevention Framework Approach (ESFA): effects after 24 and 30 months, Health Education Research, 21, 116-132.

77. De VRies, H., Mudde, A., Kremers, S. et al. (2003) The European Smoking Prevention Framework Approach (ESFA): short-term effects., Health Education Research, 18, 649-663.

78. Dijkstra, M., Mesters, I., De VRies, H., van Breukelen, G. \& Parcel, G. S. (1999) Effectiveness of a social influence approach and boosters to smoking prevention, Health Education Research, 14, 791-802. 
79. Kremers, S. P. J., Mudde, A. N. \& de VRIeS, H. (2001) Subtypes within the precontemplation stage of adolescent smoking acquisition, Addictive Behaviors., 26, 237-251.

80. Kremers, S. P. J., Mudde, A. N. \& de VRies, H. (2001) "Kicking the initiation": Do adolescent ex-smokers differ from other groups within the initiation continuum?, Preventive Medicine, 33, 392-401.

81. MCCALLISTER, L. \& FisHeR, C. S. (1978) A procedure for surveying personal networks, Sociological Methods \& Research, 7, 131-148.

82. MuthÉN, L. K. \& MutHÉN, B. O. (2004) Mplus User's Guide (Los Angeles, CA).

83. BeNtLER, P. M. \& YUAN, K. H. (1999) Structural equation modeling with small samples: Test statistics, Multivariate Behavioral Research, 34, 181-197.

84. WHITE, H. (1982) Maximum likelihood estimation of a misspecified model, Econometrica, 50, 1-25.

85. HalR, J. F., ANDERSON, R. E., TAtHAM, R. L. \& BLACK, W. C. (1998) Multivariate Data Analysis (Upper Saddle River, NJ., Prentice-Hall).

86. DOLCINI, M. M., ADLER, N. E. \& GINSBERG, D. (1996) Factors influencing agreement between self-reports and biological measures of smoking among adolescents, Journal of Research on Adolescence, 6, 515-42.

87. Harakeh, Z., SCholte, R. H. J., De VRies, H. \& Engels, R. (2005) Parental rules and communication: their association with adolescent smoking, Addiction, 100, 862-870.

88. Cairns, R. B., Leung, M. C., BuChanan, L. \& CaIRns, B. D. (2003) Friendships and social networks in childhood and adolescence: fluidity, reliability, and interrelations, Child Development, 66, 1330-1345.

89. Steglich, C., SNiJders, T. A. B. \& WeSt, P. (2006) Applying SIENA: An illustrative analysis of the co-evolution of adolescents' friendship networks, taste in music, and alcohol consumption., Methodology: Journal of Research Methods for the Behavioral and Social Sciences, 2, 45-56.

90. PEARSON, M. \& WeSt, P. (2003) Drifting Smoke Rings: Social Network Analysis and Markov Processes in a Longitudinal Study of Friendship Groups and Risk-taking, Connections, 25, 59-76.

91. DISHION, T. J. \& OWEN, L. D. (2002) A longitudinal analysis of friendship and substance use: Bidirectional influence from adolescence to adulthood, Developmental Psychology, 38, 480-491.

92. Valente, T. W., hoffman, B. R., Ritt-Olson, A., lichtman, K. \& Johnson, A. (2003) Effects of a social-network method for group assignment strategies on peer- 
led tobacco prevention programs in schools, American Journal of Public Health, 93, 1837-43.

93. WHO (World Health Organization, 2007) http://www.who.int/tobacco/health _priority/en/.

94. BYRNE, D. \& GRIFFIT, W. (1973) Interpersonal attraction, Annual Review of Psychology, 24, 317-336.

95. Harakeh, Z., Engels, R., Vermulst, A. A., De Vries, H. \& Scholte, R. H. J. (2007) The influence of best friends and siblings on adolescent smoking: a longitudinal study, Psychology and Health, 22, 269-289.

96. Mercken, L., Candel, M., Willems, P. \& De Vries, H. (2007) Distentangling social selection and social influence effects on adolescent smoking: the importance of reciprocity in friendships, Addiction, 102, 1483-1492.

97. SimOns-MORTON, B., Chen, R. S., Abroms, L. \& HAYNIE, D. L. (2004) Latent growth curve analyses of peer and parent influences on smoking progression among early adolescents, Health Psychology, 23, 612-621.

98. BROWN, B. B. (1981) A life-span approach to friendship: Age-related dimensions of an ageless relationship, Research in the Interweave of Social Rules, 2, 23-50.

99. SAtORRA, A. \& Bentler, P. M. (2001) A scaled difference chi-square test statistic for moment structure analysis, Psychometrika, 66, 507-514.

100. Aloise-Young, P. A., Graham, J. W. \& Hansen, W. B. (1994) Peer influences on smoking initiation during early adolescence: A comparison of group members and group outsiders, Journal of Applied Psychology, 79, 281-287.

101. LARSON, R. \& RICHARDS, M. H. (1991) Daily companionship in late childhood and early adolescence: changing developmental contexts, Child Development, 62, 284-300.

102. StEINBERG, L. (1988) reciprocal relation between parent-child distance and pubertal maturation, Developmental psychology, 24, 122-128.

103. Harakeh, Z., Engels, R., De VRies, H. \& Scholte, R. H. J. (2006) Correspondence between proxy and self-reports on smoking in a full family study, Drug and Alcohol Dependence, 84, 40-47.

104. Peterson, A. V., Kealy, K. A., Mann, S. L., Marek, P. M. \& Sarason, I. G. (2000) Hutchinson smoking prevention project: long-term randomized trial in schoolbased tobacco use prevention: results on smoking., Journal of the National Cancer Institute, 92, 1979-1991. 
105. KANDel, D. B., KesSler, R. C. \& MARgulies, R. Z. (1978) Antecedents of adolescent initiation into stages of drug use: a developmental analysis, Journal of Youth and Adolescence, 7, 13-40.

106. Snijders, T. A. B. \& BaerVeldt, C. (2003) A multilevel network study of the effects of delinquent behavior on friendship evolution, Journal of Mathematical Sociology, 27, 123-151.

107. Burk, W. J., Steglich, C. E. G. \& Snijders, T. A. B. (2007) Beyond dyadic interdependence: Actor-oriented models for co-evolving social networks and individual behaviors, International Journal of Behavioral Development, 31, 397-404.

108. VAN DE BUnt, G., VAN DUIJn, M. A. J. \& SNIJDERS, T. A. B. (1999) Friendship networks through time: An actor-oriented dynamic statistical network model, Computational \& Mathematical Organization Theory, 5, 167-192.

109. Snijders, T. A. B., Steglich, C. E. G., SChweinberger, M. \& Huisman, M. (2007) Manual for SIENA version 3.1. (University of Groningen: ICS; University of Oxford: Department of Statistics).

110. HUISMAN, M. \& SNIJDERS, T. A. B. (2003) Statistical analysis of longitudinal network data with changing composition, Sociological Methods \& Research, 32, 253287.

111. Hedges, L. V. \& OlKIN, I. (1985) Statistical Methods for Meta-analysis (New York, Academic Press).

112. COCHRAN, W. G. (1954) The combination of estimates from different experiments, Biometrics, 10, 101-129.

113. ClifF, A. \& ORD, J. K. (1981) Spatial Processes: Models and Applications (London, Pion).

114. MCAlister, A. L., Krosnick, J. A. \& Milburn, M. A. (1984) Causes of adolescent cigarette smoking: tests of a structural equation model, Social Psychology Quarterly, 47, 24-36.

115. VAlente, T. W., UnGer, J. B. \& ANDERSON JOHnSON, C. (2005) Do popular students smoke? The association between popularity and smoking among middle school students, Journal of Adolescent Health, 37, 323-329.

116. Campbell, R., Starkey, F., Holliday, J. et al. (2008) An informal school-based peer-led intervention for smoking prevention in adolescence (ASSIST): a cluster randomised trial, The Lancet, 371, 1595-1602. 
117. Audrey, S., Holliday, J. \& Campbell, R. (2006) It's good to talk: Adolescent perspectives of an informal, peer-led intervention to reduce smoking, Social Science \& Medicine, 63, 320-334.

118. NiCHTER, M. (2003) smoking: what does culture have to do with it?, Addiction, 98, 139-145.

119. Warren, C. W., Jones, N. R., ERiKSen, M. P. \& Asma, S. (2006) Patterns of global tobacco use in young people and implications for future chronic disease burden in adults, The Lancet, 367, 749-753.

120. OfFICE FOr National Statistics (1997) Living in Britain: Preliminary results from the 1996 General Household Survey. (London, The Stationary Office).

121. EzZATI, M. \& LOPEZ, A. D. (2003) Estimates of global mortality attributable to smoking in 2000, The Lancet, 362, 847-852.

122. DOREIAN, P. (1989) Network autocorrelation models: Problems and prospects, in: Griffith, D. A. (Ed.) Spatial Statistics: past, Present, Future (Ann Arbor, Michigan Document Services).

123. Steglich, C., Snijders, T. A. B. \& Pearson, M. (2009) Dynamic networks and Behavior: Separating Selection from Influence, (submitted for publication).

124. Mercken, L., CANDel, M., Willems, P. \& De VRIeS, H. (2009) Social influence and selection effects in the context of smoking behavior: changes during early and mid adolescence, Health Psychology, 28, 73-82.

125. Snijders, T. A. B., VAN De Bunt, G. G. \& Steglich, C. E. G. (2009) Introduction to Actor-Based Models for Network Dynamics, Social Networks, (In press).

126. Mercken, L., Snijders, T. A. B., Steglich, C. \& De VRIes, H. (2009) Dynamics of adolescent friendship networks and smoking behavior: Social network analyses in six European countries, (manuscript submitted for publication).

127. SCHWEINBERGER, M. (2008) Statistical modeling of digraph panel data: Goodness-of-fit., (Manuscript submitted for publication).

128. Mercken, L., Candel, M., Willems, P. \& De VRies, H. (2008) Social influence and selection effects in the context of smoking behavior: changes during early and mid adolescence, Health Psychology, 28, 73-82.

129. Grogan, S., Conner, M., Fry, G., Gough, B. \& Higgins, A. (2008) Gender differences in smoking: a longitudinal study of beliefs predicting smoking in 1115 year olds, Psychology and Health, ifirst, 1-18.

130. Hoving, C., Reubsaet, A. \& De Vries, H. (2007) Predictors of smoking stage transitions for adolescent boys and girls, Preventive Medicine, 44, 485-489. 
131. Hu, F. B., Flay, B. R., Hedeker, D., Siddiqui, O. \& DAY, L. E. (1995) The influences of friends' and parental smoking on adolescent smoking behavior: the effects of time and prior smoking, Journal of Applied Social Psychology, 25, 2018-2047.

132. LAWRANCE, L. (1989) Validation of a self-efficacy scale to predict adolescent smoking, Health Education Research, 4, 351-360.

133. KNeCht, A. B., BuRK, W. J., WeESIE, J. \& STeGLICH, C. (2009) Friendship and alcohol use in early adolescence: A multi-level social network approach, Journal of Research on Adolescence, (in press).

134. MCNelles, L. R. \& CONNOLLY, J. A. (1999) Intimacy between adolescent friends: Age and gender differences in intimate affect and intimate behavior, Journal of Research on Adolescence, 9, 143-159.

135. Mercken, L., Snijders, T. A. B., Steglich, C. E. G., Vartiainen, E. \& de VRies, H. (2009) Dynamics of adolescent friendship networks and smoking behavior, Social Networks, (in Press).

136. VAN HOUTTE, M. (2004) Why boys achieve less at school than girls: the difference between boys' and girls' academic culture, Educational Studies, 30, 159-173.

137. Whitelaw, S., Milosevic, L. \& Daniels, S. (2000) Gender, behaviour and achievement: a preliminary study of pupil perceptions and attitudes, Gender and Education, 12, 87-113.

138. WARrington, M., YOUNGer, M. \& Williams, J. (2000) Student attitudes, image and the gender gap, British Educational Research Journal, 26, 393-407.

139. MOUNTS, N. S. (2002) Parental management of adolescent peer relationships in context: the role of parenting style, Journal of Family Psychology, 16, 58-69.

140. WHO (2008) www.who.int/tobacco/en.

141. Engels, R., Vitaro, F., Den Exter Blokland, E., De Kemp, R. \& Scholte, R. H. J. (2004) Influence and selection processes in friendships and adolescent smoking behaviour: the role of parental smoking, Journal of Adolescence, 27, 531-544.

142. BROWN, B. B., Clasen, D. R. \& EICHER, S. A. (1986) Perceptions of peer pressure, peer conformity dispositions, and self-reported behavior among adolescents, Developmental Psychology, 22, 521-530.

143. BERNDT, T. J. (1979) Developmental changes in conformity to peers and parents, Developmental Psychology, 15, 608-616.

144. Newman, P. R. \& Newman, B. M. (1976) Early adolescence and its conflict: Group identity vs. alienation, Adolescence, 10, 127-136.

145. COterelL, J. (2007) Social networks in youth and adolescence (London, Routledge). 
146. Carlson, E. A., SROufe, L. A. \& Byron, E. (2004) The construction of experience: a longitudinal study of representation and behavior, Child Development, 75, 6683.

147. TURNER, P. J. (1991) Relations between attachment, gender, and behavior with peers in preschool, Child Development, 62, 1475-1488.

148. LIEBERMAN, M., DOYLE, A.-B. \& MARKIEWICZ, D. (1999) Developmental patterns in security of attachment to mother and father in late childhood and early adolescence: Associations with peer relations, Child Development, 70, 202213.

149. KRACKHARDT, D. (1998) Simmelian ties, super strong and sticky, in: Kramer, R. \& Neale, M. (Eds.) Power and Influence in Organizations, pp. 21-38 (Thousand Oaks, CA, Sage).

150. SIMmeL, G. (1950) Individual and society, in: Wolff, K. H. (Ed.) The sociology of Georg Simmel (Mew York, Free Press).

151. AbRAMS, D. \& HOGG, M. A. (1990) An introduction to the social identity approach, in: Abrams, D. \& Hogg, M. A. (Eds.) Social Identity Theory: Constructive and Critical Advances, pp. 1-9 (New York, Springer-Verlag).

152. ColuINS, W. A. (2003) More than myth: the developmental significance of romantic relationships during adolescence, Journal of Research on Adolescence, 13, 1-24.

153. HAMM, J. V. (2000) Do birds of a feather flock together? The variable bases for african american, asian american, and european american adolescents' selection of similar friends, Developmental Psychology, 36, 209-219.

154. TOLSON, J. M. \& URBERG, K. A. (1993) Similarity between adolescent best friends, Journal of Adolescent Research, 8, 274-288.

155. MICHELL, L. \& AMOS, A. (1997) Girls, pecking order and smoking, Social Science \& Medicine, 44, 1861-1869.

156. SIMONS-MORTON, B. G. (2002) Prospective analysis of peer and parent influences on smoking initiation among early adolescents, Prevention Science, 3, 275283.

157. Chassin, L., Presson, C., Rose, J. et al. (2005) Parenting style and smokingspecific parenting practices as predictors of adolescent smoking onset, Journal of Pediatric Psychology, 30, 333-344.

158. Engels, R. C. M. E., Finkenauer, C., KeRR, M. \& Stattin, H. (2005) Illusions of parental control: Parenting and smoking onset in Dutch and Swedish Adolescents, Journal of Applied Social Psychology, 35, 1912-1935. 
159. Fearnow, M., Chassin, L. \& Presson, C. (1998) Determinants of parental attempts to deter their children's cigarette smoking, Journal of Applied Developmental Psychology, 19, 453-468.

160. JACKSON, C. \& HeNRIKSEN, L. (1997) Do as I say: parent smoking, antismoking socialization, and smoking onset among children, Addictive Behaviors, 22, 107-114.

161. Andersen, R., Leroux, B., Bricker, J. \& Bharat Rajan, K. (2004) Antismoking parenting practices are associated with reduced rates of adolescent smoking, Archives of Pediatrics \& Adolescent Medicine, 158, 348-352.

162. Albers, A., Siegal, M., Cheng, D., Biener, L. \& Rigotti, N. (2004) Relation between local restaurant smoking regulations and attitudes towards the prevalence and social acceptability of smoking, Tobacco Control, 13, 347-355.

163. Siegel, M., Albers, A., Cheng, D., Hamilton, W. \& Biener, L. (2008) Local restaurant smoking regulations and the adolescent smoking initiation process, Archives of Pediatrics \& Adolescent Medicine, 162, 477-483.

164. Moodie, C., MACKIntosh, A., Brown, A. \& Hastings, G. (2008) Tobacco marketing awareness on youth smoking susceptibility and perceived prevalence before and after an advertising ban, European Journal of Public Health, doi:10.1093/eurpub/ckn016.

165. Davis, R., GILPIN, E., LOKen, B., VISWANATH, K. \& WaKefield, M. (2008) The role of the media in promoting and reducing tobacco use $\mathrm{NCl}$ Tobacco Control Monograph Series No. 19 (Bethesda, MD, U.S. Department of Health and Human Services, National Institutes of Health, National Cancer Institute. NIH Pub. No. 07-6242). 
DANKWOORD 



\section{DANKWOORD}

Graag wil ik een aantal mensen bedanken die ik op mijn weg ben tegengekomen.

Hein, je gaf me de vrijheid om mijn eigen weg in onderzoek te vinden. Dank je voor je grenzeloze vertrouwen, steun en enthousiasme.

Tom, het is een eer om jou hier te mogen noemen en van je te hebben mogen leren. Jouw werk(wijze) zal een bron van inspiratie voor mij blijven.

Math, jouw hulp, precisie, en kritische blik zijn uiterst waardevol geweest.

Christian, jouw expertise, geduld en onze boeiende gesprekken hebben mijn passie voor sociale netwerk analyse nog sterker gemaakt.

Mijn collega's, dank jullie voor de toffe samenwerking, voor alle hulp en gezelligheid.

Liesbeth, dank je voor het meedenken, je scherpe inzichten, stimulerende discussies, maar vooral voor je vriendschap!

Chris, dank je dat ook jij tijdens mijn promotie naast me wil staan. Bedankt voor de vele toffe gesprekken en pep talks.

Mijn vrienden, dank jullie dat jullie er steeds voor me zijn.

Papa, mama, en Els, jullie waren er altijd op de achtergrond, dank jullie voor jullie vertrouwen en steun!

Riccardo, het laatste plekje in dit dankwoord is gereserveerd voor jou want zonder jou stond ik hier niet. Dank je omdat je bent wie je bent, omdat je er voor me bent. 



\section{CURRICULUM VITAE}


Liesbeth Mercken was born on November 22, 1978, in Hasselt, Belgium. In 1996 she graduated from secondary school (Heilig Hartcollege in Lanaken). In 1997, she started studying psychology at Maastricht University. She graduated in august 2003 and received her master's degree in cognitive psychology. In September 2004 she started her PhD-project at the Faculty of Health, Medicine and Life Sciences at Maastricht University. In 2008 she moved to Wales and worked as a research associate for the Cardiff Institute of Society, Health, and Ethics, part of the Faculty of Social Sciences, Cardiff University. In September 2009 she returned to Maastricht University to work as a post-doctoral researcher at the Department of Health Education and Promotion, supported by the School for Public Health and Primary Care (CAPHRI).

\section{Publications in this dissertation}

Mercken, L., Candel, M., Willems, P., \& de Vries, H. (2007). Disentangling social selection and social influence effects on adolescent smoking: the importance of reciprocity in friendships. Addiction, 102 (9), 1483-1492.

Mercken, L., Candel, M., Willems, P., \& de Vries, H. (2009). Social influence and selection effects in the context of smoking behavior: changes during early and mid adolescence. Health Psychology, 28 (1), 73-82.

Mercken, L., Snijders, T., Steglich, C. \& de Vries, H. (2009). Dynamics of adolescent friendship networks and smoking behavior: Social network analyses within six European countries. Social Science \& Medicine (In press).

Mercken, L., Snijders, T., Steglich, C. \& de Vries, H. (2009). Disentangling selection and influence processes among Finnish adolescents: A social network analysis. Social Networks (In press).

Mercken, L., Snijders, T., Steglich, C. \& de Vries, H. (2009). Selection and influence processes in gender segregated friendship networks (submitted for publication). 


\section{Other publications}

De Vries, H., Candel, M., Engels R., \& Mercken, L. (2006). Challenges to the peer influence paradigm: results from 12-13 year olds from six European countries from the European Smoking Framework Approach study. Tobacco Control, 15, 33-89.

Mercken, L., Candel, M., Van Osch, L., \& de Vries, H. (2009). There's no smoke without fire: The impact of social networks and future friends on adolescent smoking behavior (submitted for publication).

Mercken, L., Holliday, J., Sinclair, P., Steglich, C., Snijders, T., \& Moore, L. (in preparation). A longitudinal network study of the social mechanisms underlying the dynamics of smoking behaviour in UK schools.

Mercken, L., Holliday, J., Steglich, C., \& Moore, L. (in preparation). Is the nature of friendship associated with smoking behaviour homogeneity?

Stinglhamber, F., De Cremer, D., \& Mercken, L. (2006). Perceived support as a mediator of the relationship between justice and trust: A multiple foci approach. Group Organization Management, 31 (4), 442 - 468.

Van Osch, L., Reubsaet, A., Lechner, L., Candel, M., Mercken, L., \& de Vries, H. (2007). Predicting parental sunscreen use: disentangling the role of action planning in the intention - behavior relationship. Psychology \& Health (published online).

\section{Conference presentations}

Mercken, L., Holliday, J., Steglich, C., \& Moore, L. (July, 2009). Is the strength of friendship associated with smoking behaviour homogeneity? Paper presented at the $5^{\text {th }}$ UK Social Network Conference. London, United Kingdom.

Mercken, L., Candel, M., Willems, P., \& de Vries, H. (August, 2007). Social selection or social influence: the importance of reciprocity in friendships. Research results presented at the 21st conference of the European Health Psychology Society. Maastricht, the Netherlands. 
Mercken, L., Snijders, T., Steglich, C. \& de Vries, H. (May, 2007). Effects of selection, influence, and popularity on adolescent smoking behavior: a social network analysis. Research results presented at the Sunbelt XXVII: The International Sunbelt Social Network Conference. Corfu, Greece.

Mercken, L., Snijders, T., Steglich, C. \& de Vries, H. (September, 2005). Why adolescents start to smoke: peer influence or peer selection. Paper presented at the 19th conference of the European Health Psychology Society. Galway, Ireland.

Mercken, L., Snijders, T., Steglich, C. \& de Vries, H. (July, 2005). Peer influence versus peer selection. Paper presented at the 37th World Congress of the International Institute of Sociology. Stockholm, Sweden. 